\title{
Error estimates for a numerical method for the compressible Navier-Stokes system on sufficiently smooth domains
}

\author{
Eduard Feireisl * $\quad$ Radim Hošek $^{\dagger} \quad$ David Maltese $\quad$ Antonín Novotný ${ }^{\ddagger}$
}

Institute of Mathematics of the Academy of Sciences of the Czech Republic Žitná 25, CZ-115 67 Praha 1, Czech Republic

Institut Mathématiques de Toulon, EA2134, University of Toulon

BP 20132, 83957 La Garde, France

\begin{abstract}
We derive an a priori error estimate for the numerical solution obtained by time and space discretization by the finite volume/finite element method of the barotropic Navier-Stokes equations. The numerical solution on a convenient polyhedral domain approximating a sufficiently smooth bounded domain is compared with an exact solution of the barotropic Navier-Stokes equations with a bounded density. The result is unconditional in the sense that there are no assumed bounds on the numerical solution. It is obtained by the combination of discrete relative energy inequality derived in [17] and several recent results in the theory of compressible Navier-Stokes equations concerning blow up criterion established in [26] and weak strong uniqueness principle established in [10].
\end{abstract}

Key words: Navier-Stokes system, finite element numerical method, finite volume numerical method, error estimates

AMS classification 35Q30, 65N12, 65N30, 76N10, 76N15, 76M10, 76M12

\section{Introduction}

We consider the compressible Navier-Stokes equations in the barotropic regime in a space-time cylinder $Q_{T}=(0, T) \times \Omega$, where $T>0$ is arbitrarily large and $\Omega \subset R^{3}$ is a bounded domain:

$$
\begin{gathered}
\partial_{t} \varrho+\operatorname{div}_{x}(\varrho \mathbf{u})=0 \\
\partial_{t}(\varrho \mathbf{u})+\operatorname{div}_{x}(\varrho \mathbf{u} \otimes \mathbf{u})+\nabla_{x} p(\varrho)=\operatorname{div}_{x} \mathbb{S}\left(\nabla_{x} \mathbf{u}\right),
\end{gathered}
$$

In equations (1.1 1.2) $\varrho=\varrho(t, x) \geq 0$ and $\mathbf{u}=\mathbf{u}(t, x) \in R^{3}, t \in[0, T), x \in \Omega$ are unknown density and velocity fields, while $\mathbb{S}$ and $p$ are viscous stress and pressure characterizing the fluid via the constitutive relations

$$
\mathbb{S}\left(\nabla_{x} \mathbf{u}\right)=\mu\left(\nabla_{x} \mathbf{u}+\nabla_{x}^{t} \mathbf{u}-\frac{2}{3} \operatorname{div}_{x} \mathbf{u I}\right), \mu>0
$$

${ }^{*}$ The research of E.F. leading to these results has received funding from the European Research Council under the European Union's Seventh Framework Programme (FP7/2007-2013)/ ERC Grant Agreement 320078. The Institute of Mathematics of the Academy of Sciences of the Czech Republic is supported by RVO:67985840.

${ }^{\dagger}$ The research of R.H. leading to these results has received funding from the European Research Council under the European Union's Seventh Framework Programme (FP7/2007-2013)/ ERC Grant Agreement 320078. The Institute of Mathematics of the Academy of Sciences of the Czech Republic is supported by RVO:67985840.

${ }^{\ddagger}$ The work of D.M. and A.N. has been supported by the MODTERCOM project within the APEX programme of the Provence-Alpes-Côte d'Azur region 


$$
p \in C^{2}(0, \infty) \cap C^{1}[0, \infty), p(0)=0, p^{\prime}(\varrho)>0 \text { for all } \varrho \geq 0, \lim _{\varrho \rightarrow \infty} \frac{p^{\prime}(\varrho)}{\varrho^{\gamma-1}}=p_{\infty}>0,
$$

where $\gamma \geq 1$.

Assumption $p^{\prime}(0)>0$ excludes constitutive laws behaving as $\varrho^{\gamma}$ as $\varrho \rightarrow 0^{+}$. Error estimate stated in Theorem 3.8 however still holds in the case $\lim _{\varrho \rightarrow 0^{+}} \frac{p^{\prime}(\varrho)}{\varrho^{\gamma-1}}>0$ at the price of some additional difficulties, see [17] for more details.

Equations (1.1 1.2) are completed with the no-slip boundary conditions

$$
\left.\mathbf{u}\right|_{\partial \Omega}=0
$$

and initial conditions

$$
\varrho(0, \cdot)=\varrho_{0}, \mathbf{u}(0, \cdot)=\mathbf{u}_{0}, \varrho_{0}>0 \text { in } \bar{\Omega} .
$$

We notice that under assumption (1.3), we may write

$$
\operatorname{div}_{x} \mathbb{S}\left(\nabla_{x} \mathbf{u}\right)=\mu \Delta \mathbf{u}+\frac{\mu}{3} \nabla_{x} \operatorname{div}_{x} \mathbf{u}
$$

The results on error estimates for numerical schemes for the compressible Navier-Stokes equations are in the mathematical literature on short supply. We refer the reader to papers of Liu [24], [25], Yovanovic [31], Gallouet et al. [17].

In [17] the authors have developed a methodology of deriving unconditional error estimates for the numerical schemes to the compressible Navier-Stokes equations (1.1 1.6) and applied it to the numerical scheme (3.5 3.7) discretizing the system on polyhedral domains. They have obtained error estimates for the discrete solution with respect to a classical solution of the system on the same (polyhedral) domain. In spite of the fact that [17] provides the first and to the best of our knowledge so far the sole error estimate for discrete solutions of a finite volume/finite element approximation to a model of compressible fluids that does not need any assumed bounds on the numerical solution itself, it has two weak points: 1) The existence of classical solutions on at least a short time interval to the compressible Navier-Stokes equations is known for smooth $C^{3}$ domains (see Valli, Zajaczkowski [28] or Cho, Choe, Kim [2]) but may not be in general true on the polyhedral domains. 2) The numerical solutions are compared with the classical exact solutions (as is usual in any previous existing mathematical literature). In this paper we address both points raised above and to a certain extent remove the limitations of the theory presented in [17].

More precisely, we generalize the result of Gallouet et al. [17, Theorem 3.1] in two directions:

(1) The physical domain $\Omega$ filled by the fluid and the numerical domain $\Omega_{h}, h>0$ approximating the physical domain do not need to coincide.

(2) If the physical domain is sufficiently smooth (at least of class $C^{3}$ ) and the $C^{3}$ - initial data satisfy natural compatibility conditions, we are able to obtain the unconditional error estimates with respect to any weak exact solution with bounded density.

As in [17, and in contrast with any other error estimate literature dealing with finite volume or mixed finite volume/finite element methods for compressible fluids (Yovanovich [31], Cancès et al [5], Eymard et al. [9], Villa, Villedieu [30], Rohde, Yovanovich [29], Gastaldo et al. [18] and others) this result does not require any assumed bounds on the discrete solution: the sole bounds needed for the result are those provided by the numerical scheme. Moreover, in contrast with [17] and with all above mentioned papers, the exact solution is solely weak solution with bounded density. This seemingly weak hypothesis is compensated by the regularity and compatibility conditions imposed on initial data that make possible a (sophisticated) bootstrapping argument showing that weak solutions with bounded density are in fact strong solutions in the class investigated in [17].

These results are achieved by using the following tools: 
(1) The technique introduced in [17] modified in order to accommodate non-zero velocity of the exact sample solution on the boundary of the numerical domain.

(2) Three fundamental recent results from the theory of compressible Navier-Stokes equations, namely

- Local in time existence of strong solutions in class (2.11 2.12) by Cho, Choe, Kim [2].

- Weak strong uniqueness principle proved in [10] (see also [14]).

- Blow up criterion for strong solutions in the class (2.11 2.12) by Sun, Wang, Zhang [26].

The three above mentioned items allow to show that the weak solution with bounded density emanating from the sufficiently smooth initial data is in fact a strong solution defined on the large time interval $[0, T)$.

(3) Bootstrapping argument using recent results on maximal regularity for parabolic systems by Danchin [8], Denk, Pruess, Hieber [3] and Krylov [21]. The last item allows to bootstrap the strong solution in the class Cho, Choe, Kim [2] to the class needed for the error estimates in [17], provided a certain compatibility condition for the initial data is satisfied.

\section{Preliminaries}

\subsection{Weak and strong solutions to the Navier-Stokes system}

We introduce the notion of the weak solution to system (1.1 1.4):

Definition 2.1 (Weak solutions). Let $\varrho_{0}: \Omega \rightarrow[0,+\infty)$ and $\boldsymbol{u}_{0}: \Omega \rightarrow \mathbb{R}^{3}$ with finite energy $E_{0}=$ $\int_{\Omega}\left(\frac{1}{2} \varrho_{0}\left|\boldsymbol{u}_{0}\right|^{2}+H\left(\varrho_{0}\right)\right) \mathrm{d} x$ and finite mass $0<M_{0}=\int_{\Omega} \varrho_{0} \mathrm{~d} x$. We shall say that the pair $(\varrho, \boldsymbol{u})$ is a weak solution to the problem (1.1)-(1.6) emanating from the initial data $\left(\varrho_{0}, \boldsymbol{u}_{0}\right)$ if:

(a) $\varrho \in C_{\text {weak }}\left([0, T] ; L^{a}(\Omega)\right)$, for a certain $a>1, \varrho \geq 0$ a.e. in $(0, T)$, and $\boldsymbol{u} \in L^{2}\left(0, T ; W_{0}^{1,2}\left(\Omega ; \mathbb{R}^{3}\right)\right)$.

(b) the continuity equation (1.1) is satisfied in the following weak sense

$$
\left.\int_{\Omega} \varrho \varphi \mathrm{d} x\right|_{0} ^{\tau}=\int_{0}^{\tau} \int_{\Omega}\left(\varrho \partial_{t} \varphi+\varrho \boldsymbol{u} \cdot \nabla_{x} \varphi\right) \mathrm{d} x \mathrm{dt}, \forall \tau \in[0, T], \forall \varphi \in C_{c}^{\infty}([0, T] \times \bar{\Omega}) .
$$

(c) $\varrho u \in C_{\text {weak }}\left([0, T] ; L^{b}\left(\Omega ; \mathbb{R}^{3}\right)\right)$, for a certain $b>1$, and the momentum equation (1.2) is satisfied in the weak sense,

$$
\begin{aligned}
& \left.\int_{\Omega} \varrho \boldsymbol{u} \cdot \varphi \mathrm{d} x\right|_{0} ^{\tau}=\int_{0}^{\tau} \int_{\Omega}\left(\varrho \boldsymbol{u} \cdot \partial_{t} \varphi+\varrho \boldsymbol{u} \otimes \boldsymbol{u}: \nabla \varphi+p(\varrho) \operatorname{div} \varphi\right) \mathrm{d} x \mathrm{dt} \\
& \quad-\int_{0}^{\tau} \int_{\Omega}\left(\mu \nabla \boldsymbol{u}: \nabla_{x} \varphi \mathrm{d} x \mathrm{dt}+(\mu+\lambda) \operatorname{div} \boldsymbol{u} \operatorname{div} \varphi\right) \mathrm{d} x \mathrm{dt}, \forall \tau \in[0, T], \forall \varphi \in C_{c}^{\infty}\left([0, T] \times \Omega ; \mathbb{R}^{3}\right) .
\end{aligned}
$$

(d) The following energy inequality is satisfied

$$
\begin{gathered}
\left.\int_{\Omega}\left(\frac{1}{2} \varrho|\boldsymbol{u}|^{2}+H(\varrho)\right) \mathrm{d} x\right|_{0} ^{\tau}+\int_{0}^{\tau} \int_{\Omega}\left(\mu|\nabla \boldsymbol{u}|^{2}+(\mu+\lambda)|\operatorname{div} \boldsymbol{u}|^{2}\right) \mathrm{d} x \mathrm{dt} \leq 0, \text { for a.a. } \tau \in(0, T) \\
\text { with } H(\varrho)=\varrho \int_{1}^{\varrho} \frac{p(z)}{z^{2}} \mathrm{~d} z
\end{gathered}
$$

Here and hereafter the symbol $\left.\int_{\Omega} g \mathrm{~d} x\right|_{0} ^{\tau}$ is meant for $\int_{\Omega} g(\tau, x) \mathrm{d} x-\int_{\Omega} g_{0}(x) \mathrm{d} x$. 
In the above definition, we tacitly assume that all the integrals in the formulas (2.1) $-(2.3)$ are defined and we recall that $C_{\text {weak }}\left([0, T] ; L^{a}(\Omega)\right)$ is the space of functions of $L^{\infty}\left([0, T] ; L^{a}(\Omega)\right)$ which are continuous as functions of time in the weak topology of the space $L^{a}(\Omega)$.

We notice that the function $\varrho \mapsto H(\varrho)$ is a solution of the ordinary differential equation $\varrho H^{\prime}(\varrho)-$ $H(\varrho)=p(\varrho)$ with the constant of integration fixed such that $H(1)=0$.

Note that the existence of weak solutions emanating from the finite energy initial data is well-known on bounded Lipschitz domains provided $\gamma>3 / 2$, see Lions [23] for 'large' values of $\gamma$, Feireisl and coauthors [13] for $\gamma>3 / 2$.

Proposition 2.1. Suppose the $\Omega \subset R^{3}$ is a bounded domain of class $C^{3}$. Let $r, V$ be a weak solution to problem (1.1 1.6) in $(0, T) \times \Omega$, originating from the initial data

$$
r_{0} \in C^{3}(\bar{\Omega}), r_{0}>0 \text { in } \bar{\Omega}
$$

$$
\mathbf{V}_{0} \in C^{3}\left(\bar{\Omega} ; R^{3}\right)
$$

satisfying the compatibility conditions

$$
\left.\mathbf{V}_{0}\right|_{\partial \Omega}=0,\left.\nabla_{x} p\left(r_{0}\right)\right|_{\partial \Omega}=\left.\operatorname{div}_{x} \mathbb{S}\left(\nabla_{x} \mathbf{V}_{0}\right)\right|_{\partial \Omega},
$$

and such that

$$
0 \leq r \leq \bar{r} \text { a.a. in }(0, T) \times \Omega .
$$

Then $r, \mathbf{V}$ is a classical solution satisfying the bounds:

$$
\|1 / r\|_{C([0, T] \times \bar{\Omega})}+\|r\|_{C^{1}([0, T] \times \bar{\Omega})}+\left\|\partial_{t} \nabla_{x} r\right\|_{C\left([0, T] ; L^{6}\left(\Omega ; R^{3}\right)\right)}+\left\|\partial_{t, t}^{2} r\right\|_{C\left([0, T] ; L^{6}(\Omega)\right)} \leq D,
$$

$$
\|\mathbf{V}\|_{C^{1}\left([0, T] \times \bar{\Omega} ; R^{3}\right)}+\|\mathbf{V}\|_{C\left([0, T] ; C^{2}\left(\bar{\Omega} ; R^{3}\right)\right)}+\left\|\partial_{t} \nabla_{x} \mathbf{V}\right\|_{C\left([0, T] ; L^{6}\left(\Omega ; R^{3 \times 3}\right)\right)}+\left\|\partial_{t, t}^{2} \mathbf{V}\right\|_{L^{2}\left(0, T ; L^{6}(\Omega)\right)} \leq D
$$

where $D$ depends on $\Omega, T, \bar{r}$, and the initial data $r_{0}, \mathbf{V}_{0}\left(\right.$ via $\left\|\left(r_{0}, \mathbf{V}_{0}\right)\right\|_{C^{3}\left(\bar{\Omega} ; R^{4}\right)}$ and $\min _{x \in \bar{\Omega}} r_{0}(x)$ ).

\section{Proof:}

The proof will be carried over in several steps.

\section{Step 1}

According to Cho, Choe, and Kim [2, problem (1.1-1.6) admits a strong solution unique in the class

$$
\begin{gathered}
r \in C\left(\left[0, T_{M}\right) ; W^{1,6}(\Omega)\right), \partial_{t} r \in C\left(\left[0, T_{M}\right) ; L^{6}(\Omega)\right), 1 / r \in L^{\infty}\left(Q_{T}\right), \\
\mathbf{V} \in C\left(\left[0, T_{M}\right] ; W^{2,2}\left(\Omega ; R^{3}\right)\right) \cap L^{2}\left(0, T_{M} ; W^{2,6}\left(\Omega ; R^{3}\right)\right), \partial_{t} \mathbf{V} \in L^{2}\left(0, T_{M} ; W_{0}^{1,2}\left(\Omega ; R^{3}\right)\right) .
\end{gathered}
$$

defined on a time interval $\left[0, T_{M}\right)$, where $T_{M}>0$ is finite or infinite and depends on the initial data. Moreover, for any $T_{M}^{*}<T_{M}$, there is a constant $c=c\left(T_{M}^{*}\right)$ such that

$$
\begin{gathered}
\|r\|_{L^{\infty}\left(0, T_{M}^{*} ; W^{1,6}(\Omega)\right)}+\left\|\partial_{t} r\right\|_{L^{\infty}\left(0, T_{M}^{*} ; L^{6}(\Omega)\right)}+\|1 / r\|_{L^{\infty}\left(Q_{T}\right)} \\
+\|\mathbf{V}\|_{L^{\infty}\left(0, T_{M}^{*} ; W^{2,2}\left(\Omega ; R^{3}\right)\right)}+\|\mathbf{V}\|_{L^{2}\left(0, T_{M}^{*} ; W^{2,6}\left(\Omega ; R^{3}\right)\right)}+\left\|\partial_{t} \mathbf{V}\right\|_{L^{2}\left(0, T_{M}^{*} ; W^{1,2}(\Omega)\right)} \\
\leq c\left(\left\|r_{0}\right\|_{W^{1,6}(\Omega)}+\left\|\mathbf{V}_{0}\right\|_{W^{2,2}(\Omega)}\right) .
\end{gathered}
$$

\section{Step 2}

By virtue of the weak-strong uniqueness result stated in [10, Theorem 4.1] (see also [14, Theorem 4.6]), the weak solution $r, \mathbf{V}$ coincides on the time interval $\left[0, T_{M}\right)$ with the strong solution, the existence 
of which is claimed in the previous step. According to Sun, Wang, Zhang [26, Theorem 1.3], if $T_{M}<\infty$ then

$$
\limsup _{t \rightarrow T_{M}-}\|r(t)\|_{L^{\infty}(\Omega)}=\infty .
$$

Since (2.8) holds, we infer that $T_{M}=T$. At this point we conclude that couple $(r, \mathbf{V})$ possesses regularity (2.11 2.12) and that that the bound (2.13) holds with $c$ dependent solely on $T$.

\section{Step 3}

Since the initial data enjoy the regularity and compatibility conditions stated in (2.5]2.7), a straightforward bootstrap argument gives rise to better bounds, specifically, the solution belongs to the ValliZajaczkowski (see [28, Theorem 2.5]) class

$$
\begin{gathered}
r \in C\left([0, T] ; W^{3,2}(\Omega)\right), \partial_{t} r \in L^{2}\left(0, T ; W^{2,2}(\Omega)\right), \\
\mathbf{V} \in C\left([0, T] ; W^{3,2}(\Omega)\right) \cap L^{2}\left(0, T ; W^{4,2}\left(\Omega ; R^{3}\right)\right), \partial_{t} \mathbf{V} \in L^{2}\left(0, T ; W^{2,2}\left(\Omega ; R^{3}\right)\right),
\end{gathered}
$$

where, similarly to the previous step, the norms depend only on the initial data, $\bar{r}$, and $T$.

\section{Step 4}

We write equation (1.2) in the form

$$
\partial_{t} \mathbf{V}-\frac{1}{r} \operatorname{div}_{x} \mathbb{S}\left(\nabla_{x} \mathbf{V}\right)=-\mathbf{V} \cdot \nabla_{x} \mathbf{V}+\frac{1}{r} \nabla_{x} p(r),
$$

where, by virtue of (2.15) and a simple interpolation argument, $\mathbf{V} \in C^{1+\nu}\left([0, T] \times \bar{\Omega} ; R^{3 \times 3}\right)$, and, by the same token $r \in C^{1+\nu}([0, T] \times \bar{\Omega})$ for some $\nu>0$. Consequently, by means of the standard theory of parabolic equations, see for instance Ladyzhenskaya et al. [22], we may infer that $r, \mathbf{V}$ is a classical solution,

$$
\partial_{t} \mathbf{V}, \nabla_{x}^{2} \mathbf{V} \text { Hölder continuous in }[0, T] \times \bar{\Omega} \text {. }
$$

and, going back to (1.1),

$$
\partial_{t} r \text { Hölder continuous in }[0, T] \times \bar{\Omega} \text {. }
$$

\section{Step 5}

We write

$$
\nabla_{x} \partial_{t} r=-\nabla_{x} \mathbf{V} \cdot \nabla_{x} r-\mathbf{V} \cdot \nabla_{x}^{2} r-\nabla_{x} r \operatorname{div}_{x} \mathbf{V}-r \nabla_{x} \operatorname{div}_{x} \mathbf{V}
$$

whence, by virtue (2.14), (2.17), (2.18), and the Sobolev embedding $W^{1,2} \hookrightarrow L^{6}$,

$$
\partial_{t} r \in C\left([0, T] ; W^{1,6}(\Omega)\right) .
$$

Next, we differentiate (2.16) with respect to $t$. Denoting $\mathbf{Z}=\partial_{t} \mathbf{V}$ we therefore obtain

$$
\partial_{t} \mathbf{Z}-\frac{1}{r} \operatorname{div}_{x} \mathbb{S}\left(\nabla_{x} \mathbf{Z}\right)+\mathbf{V} \cdot \nabla_{x} \mathbf{Z}=\partial_{t}\left(\frac{1}{r}\right) \operatorname{div}_{x} \mathbb{S}\left(\nabla_{x} \mathbf{V}\right)-\partial_{t} \mathbf{V} \cdot \nabla_{x} \mathbf{V}+\partial_{t}\left(\frac{1}{r} \nabla_{x} p(r)\right),
$$

where, in view of (2.19) and the previously established estimates, the expression on the right-hand side is bounded in $C\left([0, T] ; L^{6}\left(\Omega ; R^{3}\right)\right)$. Thus using the $L^{p}$-maximal regularity (see Denk, Hieber, and Pruess [3], Krylov [21] or Danchin [8, Theorem 2.2] ), we deduce that

$$
\partial_{t, t}^{2} \mathbf{V}=\partial_{t} \mathbf{Z} \in L^{2}\left(0, T ; L^{6}\left(\Omega ; R^{3}\right)\right), \partial_{t} \mathbf{V}=\mathbf{Z} \in C\left([0, T] ; W^{1,6}\left(\Omega ; R^{3}\right)\right) .
$$

Finally, writing

$$
\partial_{t, t}^{2} r=-\partial_{t} \mathbf{V} \cdot \nabla_{x} r-\mathbf{V} \cdot \partial_{t} \nabla_{x} r-\partial_{t} r \operatorname{div}_{x} \mathbf{V}-r \partial_{t} \operatorname{div}_{x} \mathbf{V}
$$

and using (2.19), (2.21), we obtain the desired conclusion

$$
\partial_{t, t}^{2} r \in C\left([0, T] ; L^{6}(\Omega)\right)
$$

Here and hereafter, we shall use notation $a \lesssim b$ and $a \approx b$. the symbol $a \lesssim b$ means that there exists $c=c(\Omega, T, \mu, \gamma)>0$ such that $a \leq c b ; a \approx b$ means $a \lesssim b$ and $b \lesssim a$. 


\subsection{Extension lemma}

Lemma 2.1. Under the hypotheses of Proposition 2.1, the functions $r$ and $\mathbf{V}$ can be extended outside $\Omega$ in such a way that:

(1) The extended functions (still denoted by $r$ and $\mathbf{V}$ ) are such that $\mathbf{V}$ is compactly supported in $[0, T] \times \mathbb{R}^{3}$ and $r \geq \underline{r}>0$.

(2)

$$
\begin{aligned}
& \|\mathbf{V}\|_{C^{1}\left([0, T] \times R^{3} ; R^{3}\right)}+\|\mathbf{V}\|_{C\left([0, T] ; C^{2}\left(R^{3} ; R^{3}\right)\right)}+\left\|\partial_{t} \nabla_{x} \mathbf{V}\right\|_{C\left([0, T] ; L^{6}\left(R^{3} ; R^{3 \times 3}\right)\right)}+\left\|\partial_{t, t}^{2} \mathbf{V}\right\|_{L^{2}\left(0, T ; L^{6}\left(R^{3}\right)\right)} \\
& \quad \lesssim\|\mathbf{V}\|_{C^{1}\left([0, T] \times \bar{\Omega} ; R^{3}\right)}+\|\mathbf{V}\|_{C\left([0, T] ; C^{2}\left(\bar{\Omega} ; R^{3}\right)\right)}+\left\|\partial_{t} \nabla_{x} \mathbf{V}\right\|_{C\left([0, T] ; L^{6}\left(\Omega ; R^{3 \times 3}\right)\right)}+\left\|\partial_{t, t}^{2} \mathbf{V}\right\|_{L^{2}\left(0, T ; L^{6}(\Omega)\right)}
\end{aligned}
$$

(3)

$$
\begin{gathered}
\|r\|_{C^{1}\left([0, T] \times R^{3}\right)}+\left\|\partial_{t} \nabla_{x} r\right\|_{C\left([0, T] ; L^{6}\left(R^{3} ; R^{3}\right)\right)}+\left\|\partial_{t, t}^{2} r\right\|_{C\left([0, T] ; L^{6}\left(R^{3}\right)\right)} \\
\lesssim\|r\|_{C^{1}([0, T] \times \bar{\Omega})}+\left\|\partial_{t} \nabla_{x} r\right\|_{C\left([0, T] ; L^{6}\left(\Omega ; R^{3}\right)\right)}+\left\|\partial_{t, t}^{2} r\right\|_{C\left([0, T] ; L^{6}(\Omega)\right)}+ \\
\|\mathbf{V}\|_{C^{1}\left([0, T] \times \bar{\Omega} ; R^{3}\right)}+\|\mathbf{V}\|_{C\left([0, T] ; C^{2}\left(\bar{\Omega} ; R^{3}\right)\right)}+\left\|\partial_{t} \nabla_{x} \mathbf{V}\right\|_{C\left([0, T] ; L^{6}\left(\Omega ; R^{3 \times 3}\right)\right)}+\left\|\partial_{t, t}^{2} \mathbf{V}\right\|_{L^{2}\left(0, T ; L^{6}(\Omega)\right)}
\end{gathered}
$$

$$
\partial_{t} r+\operatorname{div}_{x}(r \mathbf{V})=0 \text { in }(0, T) \times R^{3} .
$$

Proof: We first construct the extension of the vector field $\mathbf{V}$. To this end, we follow the standard construction in the flat domain, see Adams [1, Chapter 5, Theorem 5.22] and combine it with the standard procedure of 'flattening' of the boundary and the partition of unity technique, we get (2.22) Once this is done, we solve on the whole space the transport equation (2.24). It is easy to show that the unique solution $r$ of this equation possesses regularity and estimates stated in (2.23).

Remark 2.1. Here and hereafter, we denote $X_{T}\left(\mathbb{R}^{3}\right)$ a subset of $L^{2}\left((0, T) \times \mathbb{R}^{3}\right)$ of couples $(r, \mathbf{V}), r>0$ with finite norm

$$
\begin{gathered}
\|(r, \mathbf{V})\|_{X_{T}\left(\mathbb{R}^{3}\right)} \equiv\|r\|_{C^{1}\left([0, T] \times R^{3}\right)}+\left\|\partial_{t} \nabla_{x} r\right\|_{C\left([0, T] ; L^{6}\left(R^{3} ; R^{3}\right)\right)}+\left\|\partial_{t, t}^{2} r\right\|_{C\left([0, T] ; L^{6}\left(R^{3}\right)\right)} \\
\|\mathbf{V}\|_{C^{1}\left([0, T] \times R^{3} ; R^{3}\right)}+\|\mathbf{V}\|_{C\left([0, T] ; C^{2}\left(R^{3} ; R^{3}\right)\right)}+\left\|\partial_{t} \nabla_{x} \mathbf{V}\right\|_{C\left([0, T] ; L^{6}\left(R^{3} ; R^{3 \times 3}\right)\right)}+\left\|\partial_{t, t}^{2} \mathbf{V}\right\|_{L^{2}\left(0, T ; L^{6}\left(R^{3}\right)\right)}
\end{gathered}
$$

We notice that the first component of the couple belonging to $X_{T}\left(\mathbb{R}^{3}\right)$ is always strictly positive on $[0, T] \times R^{3}$ and set

$$
0<\underline{r}=\min _{(t, x) \in[0, T] \times \mathbb{R}^{3}} r(t, x), \quad \bar{r}=\max _{(t, x) \in[0, T] \times \mathbb{R}^{3}} r(t, x)<\infty
$$

\subsection{Physical domain, mesh approximation}

The physical space is represented by a bounded domain $\Omega \subset R^{3}$ of class $C^{3}$. The numerical domains $\Omega_{h}$ are polyhedral domains,

$$
\bar{\Omega}_{h}=\cup_{K \in \mathcal{T} K},
$$

where $\mathcal{T}$ is a set of tetrahedra which have the following property: If $K \cap L \neq \emptyset, K \neq L$, then $K \cap L$ is either a common face, or a common edge, or a common vertex. By $\mathcal{E}(K)$, we denote the set of the faces $\sigma$ of the element $K \in \mathcal{T}$. The set of all faces of the mesh is denoted by $\mathcal{E}$; the set of faces included in the boundary $\partial \Omega_{h}$ of $\Omega_{h}$ is denoted by $\mathcal{E}_{\text {ext }}$ and the set of internal faces (i.e $\mathcal{E} \backslash \mathcal{E}_{\text {ext }}$ ) is denoted by $\mathcal{E}_{\text {int }}$.

Further, we ask

$$
\mathcal{V}_{h} \in \partial \Omega_{h} \text { a vertex } \Rightarrow \mathcal{V}_{h} \in \partial \Omega \text {. }
$$


Furthermore, we suppose that each $K$ is a tetrahedron such that

$$
\xi[K] \approx \operatorname{diam}[K] \approx h,
$$

where $\xi[K]$ is the radius of the largest ball contained in $K$.

The properties of this mesh needed in the sequel are formulated in the following lemma, whose (easy) proof is left to the reader.

Lemma 2.2. There exists a positive constant $d_{\Omega}$ depending solely on the geometric properties of $\partial \Omega$ such that

$$
\operatorname{dist}[x, \partial \Omega] \leq d_{\Omega} h^{2}
$$

for any $x \in \partial \Omega_{h}$. Moreover,

$$
\left|\left(\Omega_{h} \backslash \Omega\right) \cup\left(\Omega \backslash \Omega_{h}\right)\right| \lesssim h^{2} .
$$

We find important to emphasize that $\Omega_{h} \not \subset \Omega$, in general.

\subsection{Numerical spaces}

We denote by $Q_{h}\left(\Omega_{h}\right)$ the space of piecewise constant functions:

$$
Q_{h}\left(\Omega_{h}\right)=\left\{q \in L^{2}\left(\Omega_{h}\right) \mid \forall K \in \mathcal{T}, q_{\mid K} \in \mathbb{R}\right\} .
$$

For a function $v$ in $C\left(\bar{\Omega}_{h}\right)$, we set

$$
v_{K}=\frac{1}{|K|} \int_{K} v \mathrm{~d} x \text { for } K \in \mathcal{T} \text { and } \Pi_{h}^{Q} v(x)=\sum_{K \in \mathcal{T}} v_{K} 1_{K}(x), x \in \Omega .
$$

Here and in what follows, $1_{K}$ is the characteristic function of $K$.

We define the Crouzeix-Raviart space with 'zero traces':

$$
\begin{gathered}
V_{h, 0}\left(\Omega_{h}\right)=\left\{v \in L^{2}\left(\Omega_{h}\right), \forall K \in \mathcal{T}, v_{\mid K} \in \mathbb{P}_{1}(K),\right. \\
\left.\forall \sigma \in \mathcal{E}_{\text {int }}, \sigma=K \mid L, \int_{\sigma} v_{\mid K} \mathrm{~d} S=\int_{\sigma} v_{\mid L} \mathrm{~d} S, \quad \forall \sigma^{\prime} \in \mathcal{E}_{\text {ext }}, \int_{\sigma}^{\prime} v \mathrm{~d} S=0\right\},
\end{gathered}
$$

and 'with general traces'

$$
V_{h}\left(\Omega_{h}\right)=\left\{v \in L^{2}(\Omega), \forall K \in \mathcal{T}, v_{\mid K} \in \mathbb{P}_{1}(K), \forall \sigma \in \mathcal{E}_{\text {int }}, \sigma=K \mid L, \int_{\sigma} v_{\mid K} \mathrm{~d} S=\int_{\sigma} v_{\mid L} \mathrm{~d} S\right\} .
$$

We denote by $\Pi_{h}^{V}$ the standard Crouzeix-Raviart projection, and $\Pi_{h, 0}^{V}$ the Crouzeix-Raviart projection with 'zero trace', specifically,

$$
\Pi_{h}^{V}: C\left(\bar{\Omega}_{h}\right) \rightarrow V_{h}\left(\Omega_{h}\right), \int_{\sigma} \Pi_{h}^{V}[\phi] \mathrm{dS}_{x}=\int_{\sigma} \phi \mathrm{dS}_{x} \text { for all } \sigma \in \mathcal{E}
$$

$\Pi_{h, 0}^{V}: C\left(\bar{\Omega}_{h}\right) \rightarrow V_{h}\left(\Omega_{h}\right), \int_{\sigma} \Pi_{h, 0}^{V}[\phi] \mathrm{d} \mathrm{S}_{x}=\int_{\sigma} \phi \mathrm{d} \mathrm{S}_{x}$ for all $\sigma \in \mathcal{E}_{\text {int }}, \quad \int_{\sigma} \Pi_{h, 0}^{V}[\phi] \mathrm{d} \mathrm{S}_{x}=0$ whenever $\sigma \in \mathcal{E}_{\text {ext }}$.

If $v \in W^{1,1}\left(\Omega_{h}\right)$, we set

$$
v_{\sigma}=\frac{1}{|\sigma|} \int_{\sigma} v \mathrm{~d} S \text { for } \sigma \in \mathcal{E}
$$

Each element $v \in V_{h}\left(\Omega_{h}\right)$ can be written in the form

$$
v(x)=\sum_{\sigma \in \mathcal{E}} v_{\sigma} \varphi_{\sigma}(x), \quad x \in \Omega_{h},
$$


where the set $\left\{\varphi_{\sigma}\right\}_{\sigma \in \mathcal{E}} \subset V_{h}\left(\Omega_{h}\right)$ is the classical Crouzeix-Raviart basis determined by

$$
\forall\left(\sigma, \sigma^{\prime}\right) \in \mathcal{E}^{2}, \frac{1}{\left|\sigma^{\prime}\right|} \int_{\sigma^{\prime}} \varphi_{\sigma} \mathrm{d} S=\delta_{\sigma, \sigma^{\prime}}
$$

Similarly, each element $v \in V_{h, 0}\left(\Omega_{h}\right)$ can be written in the form

$$
v(x)=\sum_{\sigma \in \mathcal{E}_{\text {int }}} v_{\sigma} \varphi_{\sigma}(x), \quad x \in \Omega_{h} .
$$

We first recall in Lemmas 2.322 .7 the standard properties of the projection $\Pi_{h}^{V}$. The collection of their proofs in the requested generality can be found in the Appendix of [17] with exception of Lemma 2.8 and its Corollary 2.1. We refer to the monograph of Brezzi, Fortin [4, the Crouzeix's and Raviart's paper [6], Gallouet, Herbin, Latché [16] for the original versions of some of these proofs. We present the proof of Lemma 2.8 dealing with the comparison of projections $\Pi_{h}^{V}$ and $\Pi_{h, 0}^{V}$ that we did not find in the literature.

Lemma 2.3. The following estimates hold true:

$$
\left\|\Pi_{h}^{V}[\phi]\right\|_{L^{\infty}(K)}+\left\|\Pi_{h, 0}^{V}[\phi]\right\|_{L^{\infty}(K)} \lesssim\|\phi\|_{L^{\infty}(K)},
$$

for all $K \in \mathcal{T}$ and $\phi \in C(K)$;

$$
\left\|\phi-\Pi_{h}^{V}[\phi]\right\|_{L^{p}(K)} \lesssim h^{s}\left\|\nabla^{s} \phi\right\|_{L^{p}\left(K ; \mathbb{R}^{d^{s}}\right)}, s=1,2,1 \leq p \leq \infty,
$$

and

$$
\left\|\nabla\left(\phi-\Pi_{h}^{V}[\phi]\right)\right\|_{L^{p}\left(K ; \mathbb{R}^{d}\right)} \leq c h^{s-1}\left\|\nabla^{s} \phi\right\|_{L^{p}\left(K ; \mathbb{R}^{d s}\right)}, s=1,2,1 \leq p \leq \infty,
$$

for all $K \in \mathcal{T}$ and $\phi \in C^{s}(K)$.

Lemma 2.4. Let $1 \leq p<\infty$. Then

$$
\sum_{\sigma \in \mathcal{E}}|\sigma| h\left|v_{\sigma}\right|^{p} \approx\|v\|_{L^{p}\left(\Omega_{h}\right)}^{p}
$$

with any $v \in V_{h}\left(\Omega_{h}\right)$.

Lemma 2.5. The following Sobolev-type inequality holds true:

$$
\|v\|_{L^{6}\left(\Omega_{h}\right)}^{2} \lesssim \sum_{K \in \mathcal{T}} \int_{K}\left|\nabla_{x} v\right|^{2} \mathrm{~d} x
$$

with any $v \in V_{h, 0}\left(\Omega_{h}\right)$.

Lemma 2.6. There holds:

$$
\sum_{K \in \mathcal{T}} \int_{K} q \operatorname{div} \Pi_{h}^{V}[\boldsymbol{v}] \mathrm{d} x=\int_{\Omega} q \operatorname{div} \boldsymbol{v} \mathrm{d} x
$$

for all $\boldsymbol{v} \in C^{1}\left(\bar{\Omega}_{h}, \mathbb{R}^{d}\right)$ and all $q \in Q_{h}\left(\Omega_{h}\right)$.

Lemma 2.7 (Jumps over faces in the Crouzeix-Raviart space). For all $v \in V_{h, 0}\left(\Omega_{h}\right)$ there holds

$$
\sum_{\sigma \in \mathcal{E}} \frac{1}{h} \int_{\sigma}[v]_{\sigma, n_{\sigma}}^{2} \mathrm{~d} S \lesssim \sum_{K \in \mathcal{T}} \int_{K}\left|\nabla_{x} v\right|^{2} \mathrm{~d} x
$$

where $[v]_{\sigma, n_{\sigma}}$ is a jump of $v$ with respect to a normal $\boldsymbol{n}_{\sigma}$ to the face $\sigma$,

$$
\forall x \in \sigma=K \mid L \in \mathcal{E}_{\mathrm{int}}, \quad[v]_{\sigma, \boldsymbol{n}_{\sigma}}(x)=\left\{\begin{array}{c}
\left.v\right|_{K}(x)-\left.v\right|_{L}(x) \text { if } \boldsymbol{n}_{\sigma}=\boldsymbol{n}_{\sigma, K} \\
\left.v\right|_{L}(x)-\left.v\right|_{K}(x) \text { if } \boldsymbol{n}_{\sigma}=\boldsymbol{n}_{\sigma, L}
\end{array},\right.
$$

( $\boldsymbol{n}_{\sigma, K}$ is the normal of $\sigma$, that is outer w.r. to element $K$ ) and

$$
\forall x \in \sigma \in \mathcal{E}_{\mathrm{ext}}, \quad[v]_{\sigma, \boldsymbol{n}_{\sigma}}(x)=v(x), \text { with } \boldsymbol{n}_{\sigma} \text { an exterior normal to } \partial \Omega .
$$


We will need to compare the projections $\Pi_{h}^{V}$ and $\Pi_{h, 0}^{V}$. Clearly they coincide on 'interior' elements meaning $K \in \mathcal{T}, K \cap \partial \Omega_{h}=\emptyset$. We have the following lemma for the tetrahedra with non void intersection with the boundary.

Lemma 2.8. We have

$$
\left\|\Pi_{h}^{V}[\phi]-\Pi_{h, 0}^{V}[\phi]\right\|_{L^{\infty}(K)}+h\left\|\nabla_{x}\left(\Pi_{h}^{V}[\phi]-\Pi_{h, 0}^{V}[\phi]\right)\right\|_{L^{\infty}\left(K ; R^{3}\right)} \lesssim \sup _{\sigma \subset K \cap \partial_{\Omega_{h}}}\|\phi\|_{L^{\infty}(\sigma)} \text { if } K \in \mathcal{T}, K \cap \partial_{\Omega_{h}} \neq \emptyset,
$$

for any $\phi \in C(K)$.

Proof: We recall the Crouzeix-Raviart basis (2.36) and the fact that $\Pi_{h}^{V}$ and $\Pi_{h, 0}^{V}$ differ only in basis functions corresponding to $\sigma \in \mathcal{E}_{\text {ext }}$. We have

$$
\left\|\Pi_{h}^{V}[\phi]-\Pi_{h, 0}^{v}[\phi]\right\|_{L^{\infty}(K)} \leq\left\|\sum_{\sigma \in \mathcal{E}(K) \cap \mathcal{E}_{\text {ext }}} \varphi_{\sigma} \frac{1}{|\sigma|} \int_{\sigma} \phi \mathrm{d} S\right\|_{L^{\infty}(K)} \leq c(K) \cdot \sup _{\sigma \in \mathcal{E}(K) \cap \mathcal{E}_{\text {ext }}}\|\phi\|_{L^{\infty}(\sigma)},
$$

and

$$
\begin{gathered}
h\left\|\nabla_{x}\left(\Pi_{h}^{V}[\phi]-\Pi_{h, 0}^{V}[\phi]\right)\right\|_{L^{\infty}(K)} \leq h\left\|\sum_{\sigma \in \mathcal{E}(K) \cap \mathcal{E}_{\mathrm{ext}}} \nabla_{x} \varphi_{\sigma} \frac{1}{|\sigma|} \int_{\sigma} \phi \mathrm{d} S\right\|_{L^{\infty}(K)} \\
\leq c h \sup _{\sigma \subseteq K \cap \partial \Omega_{h}}\|\phi\|_{L^{\infty}(\sigma)}\left\|\sum_{\sigma \in \mathcal{E}(K) \cap \mathcal{E}_{\mathrm{ext}}} \nabla_{x} \varphi_{\sigma}\right\|_{L^{\infty}(K)} \cdot
\end{gathered}
$$

The proof is completed by $\left\|\sum_{\sigma \in \mathcal{E}(K) \cap \mathcal{E}_{\text {ext }}} \nabla_{x} \varphi_{\sigma}\right\|_{L^{\infty}(K)} \leq c(K) h^{-1}$.

In fact, in the derivation of the error estimates we will use the consequence of the above observations formulated in the following two corollaries.

Corollary 2.1. Let $\phi \in C^{1}\left(R^{3}\right)$ such that $\left.\phi\right|_{\partial \Omega}=0$. Then we have,

$$
\begin{gathered}
\left\|\Pi_{h}^{V}[\phi]-\Pi_{h, 0}^{V}[\phi]\right\|_{L^{\infty}(K)}=0 \text { if } K \in \mathcal{T}, K \cap \partial_{\Omega_{h}}=\emptyset, \\
\left\|\Pi_{h}^{V}[\phi]-\Pi_{h, 0}^{V}[\phi]\right\|_{L^{\infty}(K)}+h\left\|\nabla_{x}\left(\Pi_{h}^{V}[\phi]-\Pi_{h, 0}^{V}[\phi]\right)\right\|_{L^{\infty}\left(K ; R^{3}\right)} \lesssim h^{2}\left\|\nabla_{x} \phi\right\|_{L^{\infty}\left(R^{3} ; R^{3}\right)},
\end{gathered}
$$

if $K \in \mathcal{T}_{h}, K \cap \partial \Omega_{h} \neq \emptyset, \partial K \not \subset \partial \Omega$.

Proof: Relation (2.47) follows immediately from (2.45), as there is an empty sum on the right hand side for 'interior' elements $\left(K \cap \partial \Omega_{h}=\emptyset\right)$.

For any $x \in \partial \Omega_{h}$ there exists $y \in \partial \Omega$ (and thus $\phi(y)=0$ ) such that

$$
|\phi(x)| \leq \operatorname{dist}[x, y]\left\|\nabla_{x} \phi\right\|_{L^{\infty}\left(R^{3} ; R^{3}\right)} \lesssim h^{2}\left\|\nabla_{x} \phi\right\|_{L^{\infty}\left(R^{3} ; R^{3}\right)},
$$

where we used Lemma 2.2 for the latter inequality. The proof is completed by taking supremum over $K \in \mathcal{T}_{h}$ and combining with (2.49). Note that the mesh regularity property (2.29) supplies a uniform estimate of constants $c(K)$ from the previous lemma, which enables to write the latter inequality in (2.49).

Corollary 2.2. For any $\phi \in C\left(R^{3}\right)$,

$$
\left\|\Pi_{h}^{V}[\phi]-\Pi_{h, 0}^{V}[\phi]\right\|_{L^{p}(K)} \lesssim h^{3 / p}\|\phi\|_{L^{\infty}\left(\bar{\Omega}_{h}\right)}, 1 \leq p<\infty .
$$


Proof: Apply inverse estimates (see e.g. [20, Lemma 2.9]) to (2.45).

We will frequently use the Poincaré, Sobolev and interpolation inequalities on tetrahedra reported in the following lemma.

\section{Lemma 2.9.}

(1) We have,

$$
\begin{gathered}
\left\|v-v_{K}\right\|_{L^{p}(K)} \lesssim h\|\nabla v\|_{L^{p}(K)}, \\
\forall \sigma \in \mathcal{E}(K),\left\|v-v_{\sigma}\right\|_{L^{p}(K)} \lesssim h\|\nabla v\|_{L^{p}(K)},
\end{gathered}
$$

for any $v \in W^{1, p}(K)$, where $1 \leq p \leq \infty$.

(2) There holds

$$
\begin{gathered}
\left\|v-v_{K}\right\|_{L^{p^{*}}(K)} \lesssim\|\nabla v\|_{L^{p}(K)}, \\
\forall \sigma \in \mathcal{E}(K),\left\|v-v_{\sigma}\right\|_{L^{p^{*}}(K)} \lesssim\|\nabla v\|_{L^{p}(K)},
\end{gathered}
$$

for any $v \in W^{1, p}(K), 1 \leq p<d$, where $p^{*}=\frac{d p}{d-p}$.

(3) We have,

$$
\begin{aligned}
& \left\|v-v_{K}\right\|_{L^{q}(K)} \leq c h^{\beta}\|\nabla v\|_{L^{p}\left(K ; \mathbb{R}^{d}\right)}, \\
& \left\|v-v_{\sigma}\right\|_{L^{q}(K)} \leq c h^{\beta}\|\nabla v\|_{L^{p}\left(K ; \mathbb{R}^{d}\right)},
\end{aligned}
$$

for any $v \in W^{1, p}(K), 1 \leq p<d$, where $\frac{1}{q}=\frac{\beta}{p}+\frac{1-\beta}{p^{*}}$.

We finish the section of preliminaries by recalling two algebraic inequalities 1) the 'imbedding' inequality

$$
\left(\sum_{i=1}^{L}\left|a_{i}\right|^{p}\right)^{1 / p} \leq\left(\sum_{i=1}^{L}\left|a_{i}\right|^{q}\right)^{1 / q}
$$

for all $a=\left(a_{1}, \ldots, a_{L}\right) \in \mathbb{R}^{L}, 1 \leq q \leq p<\infty$ and the discrete Hölder inequality

$$
\sum_{i=1}^{L}\left|a_{i}\right|\left|b_{i}\right| \leq\left(\sum_{i=1}^{L}\left|a_{i}\right|^{q}\right)^{1 / q}\left(\sum_{i=1}^{L}\left|a_{i}\right|^{p}\right)^{1 / p}
$$

for all $a=\left(a_{1}, \ldots, a_{L}\right) \in \mathbb{R}^{L}, b=\left(b_{1}, \ldots, b_{L}\right) \in \mathbb{R}^{L}, \frac{1}{q}+\frac{1}{p}=1$.

\section{Main result}

Here and hereafter we systematically use the following abbreviated notation:

$$
\hat{\phi}=\Pi_{h}^{Q}[\phi], \quad \phi_{h}=\Pi_{h}^{V}[\phi], \quad \phi_{h, 0}=\Pi_{h, 0}^{V}[\phi] .
$$

For a function $v \in C\left([0, T], L^{1}(\Omega)\right)$ we set

$$
v^{n}(x)=v\left(t_{n}, x\right)
$$

where $t_{0}=0<t_{1}<\ldots<t_{n-1}<t_{n}<t_{n+1}<\ldots t_{N}=T$ is a partition of the interval $[0, T]$. Finally, for a function $v \in V_{h}\left(\Omega_{h}\right)$ we denote

$$
\nabla_{h} v(x)=\sum_{K \in \mathcal{T}} \nabla_{x} v(x) 1_{K}(x), \operatorname{div}_{h} \mathbf{v}(x)=\sum_{K \in \mathcal{T}} \operatorname{div}_{x} \mathbf{v}(x) 1_{K}(x) .
$$


In order to ensure the positivity of the approximate densities, we shall use an upwinding technique for the density in the mass equation. For $q \in Q_{h}\left(\Omega_{h}\right)$ and $\boldsymbol{u} \in \mathbf{V}_{h, 0}\left(\Omega_{h} ; \mathbb{R}^{3}\right)$, the upwinding of $q$ with respect to $\boldsymbol{u}$ is defined, for $\sigma=K \mid L \in \mathcal{E}_{\text {int }}$ by

$$
q_{\sigma}^{\mathrm{up}}=\left\{\begin{array}{l}
q_{K} \text { if } \boldsymbol{u}_{\sigma} \cdot \boldsymbol{n}_{\sigma, K}>0 \\
q_{L} \text { if } \boldsymbol{u}_{\sigma} \cdot \boldsymbol{n}_{\sigma, K} \leq 0
\end{array},\right.
$$

and we denote

$$
\mathrm{Up}_{K}(q, \mathbf{u}) \equiv \sum_{\sigma \in \mathcal{E}(K) c \cap \mathcal{E}_{\mathrm{int}}} q_{\sigma}^{\mathrm{up}} \boldsymbol{u}_{\sigma} \cdot \mathbf{n}_{\sigma, K}=\sum_{\sigma \in \mathcal{E}(K) \cap \mathcal{E}_{\mathrm{int}}}\left(q_{K}\left[\boldsymbol{u}_{\sigma} \cdot \mathbf{n}_{\sigma, K}\right]^{+}+q_{L}\left[\boldsymbol{u}_{\sigma} \cdot \mathbf{n}_{\sigma, K}\right]^{-}\right),
$$

where $a^{+}=\max (a, 0), a^{-}=\min (a, 0)$.

\subsection{Numerical scheme}

We consider a couple $\left(\varrho^{n}, \boldsymbol{u}^{n}\right)=\left(\varrho^{n,(\Delta t, h)}, \boldsymbol{u}^{n,(\Delta t, h)}\right)$ of (numerical) solutions of the following algebraic system (numerical scheme):

$$
\varrho^{n} \in Q_{h}\left(\Omega_{h}\right), \varrho^{n}>0, \mathbf{u}^{n} \in V_{h, 0}\left(\Omega_{h} ; R^{3}\right), \quad n=0,1, \ldots, N,
$$

$$
\sum_{K \in \mathcal{T}}|K| \frac{\varrho_{K}^{n}-\varrho_{K}^{n-1}}{\Delta t} \phi_{K}+\sum_{K \in \mathcal{T}} \sum_{\sigma \in \mathcal{E}(K)}|\sigma| \varrho_{\sigma}^{n, \text { up }}\left(\mathbf{u}_{\sigma}^{n} \cdot \mathbf{n}_{\sigma, K}\right) \phi_{K}=0 \text { for any } \phi \in Q_{h}\left(\Omega_{h}\right) \text { and } n=1, \ldots, N
$$

$$
\begin{aligned}
\sum_{K \in \mathcal{T}} \frac{|K|}{\Delta t}\left(\varrho_{K}^{n} \hat{\boldsymbol{u}}_{K}^{n}-\varrho_{K}^{n-1} \hat{\boldsymbol{u}}_{K}^{n-1}\right) \cdot \boldsymbol{v}_{K}+\sum_{K \in \mathcal{T}} \sum_{\sigma \in \mathcal{E}(K)}|\sigma| \varrho_{\sigma}^{n, \text { up }} \hat{\boldsymbol{u}}_{\sigma}^{n, \text { up }}\left[\boldsymbol{u}_{\sigma}^{n} \cdot \boldsymbol{n}_{\sigma, K}\right] \cdot \boldsymbol{v}_{K} \\
\quad-\sum_{K \in \mathcal{T}} p\left(\varrho_{K}^{n}\right) \sum_{\sigma \in \mathcal{E}(K)}|\sigma| \boldsymbol{v}_{\sigma} \cdot \mathbf{n}_{\sigma, K}+\mu \sum_{K \in \mathcal{T}} \int_{K} \nabla \boldsymbol{u}^{n}: \nabla \boldsymbol{v} \mathrm{d} x \\
+\frac{\mu}{3} \sum_{K \in \mathcal{T}} \int_{K} \operatorname{div} \boldsymbol{u}^{n} \operatorname{div} \boldsymbol{v} \mathrm{d} x=0, \text { for any } \boldsymbol{v} \in V_{h, 0}\left(\Omega ; R^{3}\right) \text { and } n=1, \ldots, N
\end{aligned}
$$

The numerical solutions depend on the size $\mathrm{h}$ of the space discretisation and on the time step $\Delta t$. For the sake of clarity and in order to simplify notation we will always systematically write in all formulas $\left(\varrho^{n}, \boldsymbol{u}^{n}\right)$ instead of $\left(\varrho^{n,(\Delta t, h)}, \boldsymbol{u}^{n,(\Delta t, h)}\right)$.

Existence of a solution to problem (3.5) 3.7) is well known together with the fact that any solution $\left(\varrho^{n}\right)_{1 \leq n \leq N} \subset\left(Q_{h}(\Omega)\right)^{N}$ satisfies $\varrho^{n}>0$ provided $\varrho^{0}>0$ thanks to the upwind choice in (3.6) (see e.g. [15, 20]).

Remark 3.1. Throughout the paper, $q_{\sigma}^{\mathrm{up}}$ is defined in (3.4), where $\mathbf{u}$ is the numerical solution constructed in (3.5 3.7$)$.

\subsection{Error estimates}

The main result of this paper is announced in the following theorem:

Theorem 3.1. Let $\Omega \subset R^{3}$ be a bounded domain of class $C^{3}$ and let the pressure satisfy (1.4) with $\gamma \geq 3 / 2$. Let $\left\{\varrho^{n}, \mathbf{u}^{n}\right\}_{0 \leq n \leq N}$ be a family of numerical solutions resulting from the scheme (3.5 3.7). Moreover, suppose there are initial data $\left[r_{0}, \mathbf{V}_{0}\right]$ belonging to the regularity class specified in Proposition 
2.1 and giving rise to a weak solution $[r, \mathbf{V}]$ to the initial-boundary value problem $(1.1,1.6)$ in $(0, T) \times \Omega$ satisfying

$$
0 \leq r(t, x) \leq \bar{r} \text { a.a. in }(0, T) \times \Omega .
$$

Then $[r, \mathbf{V}]$ is regular and there exists a positive number

$$
\begin{gathered}
C=C\left(M_{0}, E_{0}, \underline{r}, \bar{r},\left|p^{\prime}\right|_{C^{1}[\underline{r}, \bar{r}]},\left\|\left(\partial_{t} r, \nabla r, \boldsymbol{V}, \partial_{t} \boldsymbol{V}, \nabla \boldsymbol{V}, \nabla^{2} \boldsymbol{V}\right)\right\|_{L^{\infty}\left(Q_{T} ; \mathbb{R}^{45}\right)},\right. \\
\left.\left\|\partial_{t}^{2} r\right\|_{L^{1}\left(0, T ; L^{\prime}(\Omega)\right)},\left\|\partial_{t} \nabla r\right\|_{L^{2}\left(0, T ; L^{6 \gamma / 5 \gamma-6}\left(\Omega ; \mathbb{R}^{3}\right)\right)},\left\|\partial_{t}^{2} \boldsymbol{V}, \partial_{t} \nabla \boldsymbol{V}\right\|_{L^{2}\left(0, T ; L^{6 / 5}\left(\Omega ; \mathbb{R}^{12}\right)\right)},\right)
\end{gathered}
$$

such that

$$
\begin{gathered}
\sup _{1 \leq n \leq N} \int_{\Omega \cap \Omega_{h}}\left[\frac{1}{2} \varrho^{n}\left|\hat{\mathbf{u}}^{n}-\mathbf{V}\left(t_{n}, \cdot\right)\right|^{2}+H\left(\varrho^{n}\right)-H^{\prime}\left(r\left(t_{n}, \cdot\right)\right)\left(\varrho^{n}-r\left(t_{n}, \cdot\right)\right)-H\left(r\left(t_{n}\right)\right)\right] \mathrm{d} x \\
+\Delta t \sum_{1 \leq n \leq N} \int_{\Omega \cap \Omega_{h}}\left|\nabla_{h} \mathbf{u}^{n}-\nabla_{x} \mathbf{V}\left(t_{n}, \cdot\right)\right|^{2} \mathrm{~d} x \\
\left.\leq C\left(\sqrt{\Delta t}+h^{a}+\int_{\Omega \cap \Omega_{h}}\left[\frac{1}{2} \varrho^{0}\left|\hat{\mathbf{u}}^{0}-\mathbf{V}_{0}\right|^{2}+H\left(\varrho^{0}\right)-H^{\prime}\left(r_{0}\right)\left(\varrho^{0}-r_{0}\right)-H\left(r_{0}\right)\right)\right] \mathrm{d} x\right),
\end{gathered}
$$

where

$$
a=\frac{2 \gamma-3}{\gamma} \text { if } \frac{3}{2} \leq \gamma \leq 2, a=\frac{1}{2} \text { otherwise. }
$$

Note that for $\gamma=3 / 2$ Theorem 3.1 gives only uniform bounds on the difference of exact and numerical solution, not the convergence.

\section{Uniform estimates}

If we take $\phi=1$ in formula (3.6) we get immediately the conservation of mass:

$$
\forall n=1, \ldots N, \quad \int_{\Omega_{h}} \varrho^{n} \mathrm{~d} x=\int_{\Omega_{h}} \varrho^{0} \mathrm{~d} x
$$

Next Lemma reports the standard energy estimates for the numerical scheme (3.5] 3.7), see again [15, 20].

Lemma 4.1. Let $\left(\varrho^{n}, \boldsymbol{u}^{n}\right)$ be a solution of the discrete problem (3.5 3.7) with the pressure $p$ satisfying (1.4). Then there exist

$$
\begin{aligned}
& \varrho_{\sigma}^{n} \in\left[\min \left(\varrho_{K}^{n}, \varrho_{L}^{n}\right), \max \left(\varrho_{K}^{n}, \varrho_{L}^{n}\right)\right], \sigma=K \mid L \in \mathcal{E}_{\text {int }}, n=1, \ldots, N, \\
& \varrho_{K}^{n-1, n} \in\left[\min \left(\varrho_{K}^{n-1}, \varrho_{K}^{n}\right), \max \left(\varrho_{K}^{n-1}, \varrho_{K}^{n}\right)\right], K \in \mathcal{T}, n=1, \ldots, N,
\end{aligned}
$$

such that

$$
\begin{aligned}
& \sum_{K \in \mathcal{T}}|K|\left(\frac{1}{2} \varrho_{K}^{m}\left|\boldsymbol{u}_{K}^{m}\right|^{2}+\right.\left.H\left(\varrho_{K}^{m}\right)\right)-\sum_{K \in \mathcal{T}}|K|\left(\frac{1}{2} \varrho_{K}^{0}\left|\boldsymbol{u}_{K}^{0}\right|^{2}+H\left(\varrho_{K}^{0}\right)\right) \\
&+\Delta t \sum_{n=1}^{m} \sum_{K \in \mathcal{T}}\left(\mu \int_{K}\left|\nabla_{x} \boldsymbol{u}^{n}\right|^{2} \mathrm{~d} x+(\mu+\lambda) \int_{K}\left|\operatorname{div} \boldsymbol{u}^{n}\right|^{2} \mathrm{~d} x\right) \\
&+\left[D_{\text {time }}^{m,|\Delta \boldsymbol{u}|}\right]+\left[D_{\text {time }}^{m,|\Delta \varrho|}\right]+\left[D_{\text {space }}^{m,|\Delta \boldsymbol{u}|}\right]+\left[D_{\text {space }}^{m, \mid \Delta \varrho}\right]=0
\end{aligned}
$$


for all $m=1, \ldots, N$, where

$$
\begin{aligned}
& {\left[D_{\text {time }}^{m,|\Delta \boldsymbol{u}|}\right]=\sum_{n=1}^{m} \sum_{K \in \mathcal{T}}|K| \varrho_{K}^{n-1} \frac{\left|\boldsymbol{u}_{K}^{n}-\boldsymbol{u}_{K}^{n-1}\right|^{2}}{2},} \\
& {\left[D_{\text {time }}^{m,|\Delta \varrho|}\right]=\sum_{n=1}^{m} \sum_{K \in \mathcal{T}}|K| H^{\prime \prime}\left(\varrho_{K}^{n-1, n}\right) \frac{\left|\varrho_{K}^{n}-\varrho_{K}^{n-1}\right|^{2}}{2},} \\
& {\left[D_{\text {space }}^{m,|\Delta u|}\right]=\Delta t \sum_{n=1}^{m} \sum_{\sigma=K \mid L \in \mathcal{E}_{\text {int }}}|\sigma| \varrho_{\sigma}^{n, \text { up }} \frac{\left(\boldsymbol{u}_{K}^{n}-\boldsymbol{u}_{L}^{n}\right)^{2}}{2}\left|\boldsymbol{u}_{\sigma}^{n} \cdot \boldsymbol{n}_{\sigma, K}\right|,} \\
& {\left[D_{\text {space }}^{m,|\Delta \varrho|}\right]=\Delta t \sum_{n=1}^{m} \sum_{\sigma=K \mid L \in \mathcal{E}_{\text {int }}}|\sigma| H^{\prime \prime}\left(\varrho_{\sigma}^{n}\right) \frac{\left(\varrho_{K}^{n}-\varrho_{L}^{n}\right)^{2}}{2}\left|\boldsymbol{u}_{\sigma}^{n} \cdot \mathbf{n}_{\sigma, K}\right| .}
\end{aligned}
$$

We have the following corollary of Lemma 4.1] (see [17, Lemma 4.1, Lemma 4.2]):

Corollary 4.1. Under assumptions of Lemma 4.1, we have:

(1) There exists $c=c\left(M_{0}, E_{0}\right)>0$ (independent of $n$, $h$ and $\Delta t$ ) such that

$$
\begin{gathered}
k \sum_{n=1}^{N} \int_{K}\left|\nabla_{x} \boldsymbol{u}^{n}\right|^{2} \mathrm{~d} x \leq c, \\
k \sum_{n=1}^{N}\left\|\boldsymbol{u}^{n}\right\|_{L^{6}\left(\Omega_{h} ; \mathbb{R}^{3}\right)}^{2} \leq c, \\
\sup _{n=0, \ldots N}\left\|\varrho^{n} \hat{\boldsymbol{u}}^{n^{2}}\right\|_{L^{1}\left(\Omega_{h}\right)} \leq c .
\end{gathered}
$$

(2)

$$
\sup _{n=0, \ldots N}\left\|\varrho^{n}\right\|_{L^{\gamma}\left(\Omega_{h}\right)} \leq c,
$$

(3) If the pair $(r, \boldsymbol{U})$ belongs to the class (2.25) there is $c=c\left(M_{0}, E_{0}, \underline{r}, \bar{r},\|\boldsymbol{U}, \nabla \boldsymbol{U}\|_{L^{\infty}\left(Q_{T} ; \mathbb{R}^{12}\right)}\right)>0$ such that for all $n=1, \ldots, N$,

$$
\sup _{n=0, \ldots N} \mathcal{E}\left(\varrho^{n}, \hat{\boldsymbol{u}}^{n} \mid \hat{r}\left(t_{n}\right), \hat{\boldsymbol{U}}\left(t_{n}\right)\right) \leq c,
$$

where

$$
\mathcal{E}(\varrho, \boldsymbol{u} \mid z, \mathbf{v})=\int_{\Omega_{h}}\left(\varrho|\boldsymbol{u}-\mathbf{v}|^{2}+E(\varrho \mid z)\right) \mathrm{d} x, \quad E(\varrho \mid z)=H(\varrho)-H^{\prime}(z)(\varrho-z)-H(z) .
$$

(4) There exists $c=c\left(M_{0}, E_{0}, \underline{r},\left|p^{\prime}\right|_{C \underline{\underline{r}}, \bar{r}]}\right)>0$ such that

$$
\begin{gathered}
\Delta t \sum_{n=1}^{m} \sum_{\sigma=K \mid L \in \mathcal{E}_{\mathrm{int}}}|\sigma|\left(\varrho_{K}^{n}-\varrho_{L}^{n}\right)^{2}\left[\frac{1_{\left\{\bar{\varrho}_{\sigma}^{n} \geq 1\right\}}}{\left[\max \left\{\varrho_{K}, \varrho_{L}\right\}\right]^{2-\gamma}}+1_{\left\{\bar{\varrho}_{\sigma}^{n}<1\right\}}\right]\left|\boldsymbol{u}_{\sigma}^{n} \cdot \mathbf{n}_{\sigma, K}\right| \leq c \quad \text { if } \gamma \in[1,2) \\
\Delta t \sum_{n=1}^{m} \sum_{\sigma=K \mid L \in \mathcal{E}_{\mathrm{int}}}|\sigma|\left(\varrho_{K}^{n}-\varrho_{L}^{n}\right)^{2}\left|\boldsymbol{u}_{\sigma}^{n} \cdot \mathbf{n}_{\sigma, K}\right| \leq c \quad \text { if } \gamma \geq 2
\end{gathered}
$$




\section{Discrete relative energy inequality}

The starting point of our error analysis is the discrete relative energy inequality derived for the numerical scheme (3.5] 3.7) in [17, Theorem 5.1].

Lemma 5.1. Let $\left(\varrho^{n}, \boldsymbol{u}^{n}\right)$ be a solution of the discrete problem (3.5 3.7) with the pressure $p$ satisfying (1.4). Then there holds for all $m=1, \ldots, N$,

$$
\begin{aligned}
\sum_{K \in \mathcal{T}} \frac{1}{2}|K|\left(\varrho_{K}^{m}\left|\boldsymbol{u}_{K}^{m}-\boldsymbol{U}_{K}^{m}\right|^{2}-\varrho_{K}^{0}\left|\boldsymbol{u}_{K}^{0}-\boldsymbol{U}_{K}^{0}\right|^{2}\right)+\sum_{K \in \mathcal{T}}|K|\left(E\left(\varrho_{K}^{m} \mid r_{K}^{m}\right)-E\left(\varrho_{K}^{0} \mid r_{K}^{0}\right)\right) \\
+\Delta t \sum_{n=1}^{m} \sum_{K \in \mathcal{T}}\left(\mu \int_{K}\left|\nabla_{x}\left(\boldsymbol{u}^{n}-\boldsymbol{U}^{n}\right)\right|^{2} \mathrm{~d} x+\frac{\mu}{3} \int_{K}\left|\operatorname{div}\left(\boldsymbol{u}^{n}-\boldsymbol{U}^{n}\right)\right|^{2} \mathrm{~d} x\right) \leq \sum_{i=1}^{6} T_{i},
\end{aligned}
$$

for any $0<r^{n} \in Q_{h}\left(\Omega_{h}\right), \boldsymbol{U}^{n} \in V_{h, 0}\left(\Omega_{h} ; \mathbb{R}^{3}\right), n=1, \ldots, N$, where

$$
\begin{aligned}
& T_{1}=\Delta t \sum_{n=1}^{m} \sum_{K \in \mathcal{T}}\left(\mu \int_{K} \nabla_{x} \boldsymbol{U}^{n}: \nabla_{x}\left(\boldsymbol{U}^{n}-\boldsymbol{u}^{n}\right) \mathrm{d} x+\frac{\mu}{3} \int_{K} \operatorname{div} \boldsymbol{U}^{n} \operatorname{div}\left(\boldsymbol{U}^{n}-\boldsymbol{u}^{n}\right) \mathrm{d} x\right), \\
& T_{2}=\Delta t \sum_{n=1}^{m} \sum_{K \in \mathcal{T}}|K| \varrho_{K}^{n-1} \frac{\boldsymbol{U}_{K}^{n}-\boldsymbol{U}_{K}^{n-1}}{\Delta t} \cdot\left(\frac{\boldsymbol{U}_{K}^{n-1}+\boldsymbol{U}_{K}^{n}}{2}-\boldsymbol{u}_{K}^{n-1}\right), \\
& T_{3}=-\Delta t \sum_{n=1}^{m} \sum_{K \in \mathcal{T}} \sum_{\substack{\sigma \in \mathcal{E}(K) \\
\sigma=K \mid L}}|\sigma| \varrho_{\sigma}^{n, \text { up }}\left(\frac{\boldsymbol{U}_{K}^{n}+\boldsymbol{U}_{L}^{n}}{2}-\hat{\boldsymbol{u}}_{\sigma}^{n, \text { up }}\right) \cdot \boldsymbol{U}_{K}^{n}\left[\boldsymbol{u}_{\sigma}^{n} \cdot \boldsymbol{n}_{\sigma, K}\right], \\
& T_{4}=-\Delta t \sum_{n=1}^{m} \sum_{K \in \mathcal{T}} \sum_{\sigma \in \mathcal{E}(K)}|\sigma| p\left(\varrho_{K}^{n}\right)\left[\boldsymbol{U}_{\sigma}^{n} \cdot \boldsymbol{n}_{\sigma, K}\right], \\
& T_{5}=\Delta t \sum_{n=1}^{m} \sum_{K \in \mathcal{T}} \frac{|K|}{\Delta t}\left(r_{K}^{n}-\varrho_{K}^{n}\right)\left(H^{\prime}\left(r_{K}^{n}\right)-H^{\prime}\left(r_{K}^{n-1}\right)\right), \\
& T_{6}=\Delta t \sum_{n=1}^{m} \sum_{K \in \mathcal{T}} \sum_{\sigma \in \mathcal{E}(K)}|\sigma| \varrho_{\sigma}^{n, \text { up }} H^{\prime}\left(r_{K}^{n-1}\right)\left[\boldsymbol{u}_{\sigma}^{n} \cdot \boldsymbol{n}_{\sigma, K}\right] .
\end{aligned}
$$

\section{Approximate discrete relative energy inequality}

In this section, we transform the right hand side of the relative energy inequality (5.1) to a form that is more convenient for the comparison with the strong solution. This transformation is given in the following lemma.

Lemma 6.1 (Approximate relative energy inequality). Let $\left(\varrho^{n}, \boldsymbol{u}^{n}\right)$ be a solution of the discrete problem (3.5 3.7), where the pressure satisfies (1.4) with $\gamma \geq 3 / 2$. Then there exists

$$
\begin{gathered}
c=c\left(M_{0}, E_{0}, \underline{r}, \bar{r},\left|p^{\prime}\right|_{C^{1}[\underline{r}, \bar{r}]},\left\|\left(\partial_{t} r, \nabla r, \boldsymbol{V}, \partial_{t} \boldsymbol{V}, \nabla \boldsymbol{V}\right)\right\|_{L^{\infty}\left(Q_{T} ; \mathbb{R}^{18}\right)},\right. \\
\left.\left\|\partial_{t}^{2} r\right\|_{L^{1}\left(0, T ; L^{\prime}(\Omega)\right)},\left\|\partial_{t} \nabla r\right\|_{L^{2}\left(0, T ; L^{6 \gamma / 5 \gamma-6}\left(\Omega ; \mathbb{R}^{3}\right)\right)}\right)>0,
\end{gathered}
$$

such that for all $m=1, \ldots, N$, we have:

$$
\begin{aligned}
& \int_{\Omega_{h}}\left(\varrho^{m}\left|\hat{\boldsymbol{u}}^{m}-\hat{\boldsymbol{V}}_{h, 0}^{m}\right|^{2}+E\left(\varrho^{m} \mid \hat{r}^{m}\right)\right) \mathrm{d} x-\int_{\Omega_{h}}\left(\varrho^{0}\left|\hat{\boldsymbol{u}}^{0}-\hat{\boldsymbol{V}}_{h, 0}^{0}\right|^{2}+E\left(\varrho^{0} \mid \hat{r}^{0}\right)\right) \mathrm{d} x \\
& +\Delta t \sum_{n=1}^{m} \sum_{K \in \mathcal{T}}\left(\mu \int_{K}\left|\nabla_{x}\left(\boldsymbol{u}^{n}-\boldsymbol{V}_{h, 0}^{n}\right)\right|^{2} \mathrm{~d} x+\frac{\mu}{3} \int_{K}\left|\operatorname{div}\left(\boldsymbol{u}^{n}-\boldsymbol{V}_{h, 0}^{n}\right)\right|^{2} \mathrm{~d} x\right) \leq \sum_{i=1}^{6} S_{i}+R_{h, \Delta t}^{m}+G^{m},
\end{aligned}
$$


for any couple $(r, \mathbf{V})$ belonging to the class (2.25), where

$$
\begin{aligned}
& S_{1}=\Delta t \sum_{n=1}^{m} \sum_{K \in \mathcal{T}}\left(\mu \int_{K} \nabla_{x} \boldsymbol{V}_{h, 0}^{n}: \nabla_{x}\left(\boldsymbol{V}_{h, 0}^{n}-\boldsymbol{u}^{n}\right) \mathrm{d} x+\frac{\mu}{3} \int_{K} \operatorname{div} \boldsymbol{V}_{h, 0}^{n} \operatorname{div}\left(\boldsymbol{V}_{h, 0}^{n}-\boldsymbol{u}^{n}\right) \mathrm{d} x\right), \\
& S_{2}=\Delta t \sum_{n=1}^{m} \sum_{K \in \mathcal{T}}|K| \varrho_{K}^{n-1} \frac{\boldsymbol{V}_{h, 0, K}^{n}-\boldsymbol{V}_{h, 0, K}^{n-1}}{\Delta t} \cdot\left(\boldsymbol{V}_{h, 0, K}^{n}-\boldsymbol{u}_{K}^{n}\right), \\
& S_{3}=\Delta t \sum_{n=1}^{m} \sum_{K \in \mathcal{T}} \sum_{\sigma \in \mathcal{E}(K)}|\sigma| \varrho_{\sigma}^{n, \text { up }}\left(\hat{\boldsymbol{V}}_{h, 0, \sigma}^{n, \text { up }}-\hat{\boldsymbol{u}}_{\sigma}^{n, \text { up }}\right) \cdot\left(\boldsymbol{V}_{h, 0, \sigma}^{n}-\boldsymbol{V}_{h, 0, K}^{n}\right) \hat{\boldsymbol{V}}_{h, 0, \sigma}^{n, \text { up }} \cdot \boldsymbol{n}_{\sigma, K}, \\
& S_{4}=-\Delta t \sum_{n=1}^{m} \int_{\Omega_{h}} p\left(\varrho^{n}\right) \operatorname{div} \boldsymbol{V}^{n} \mathrm{~d} x, \\
& S_{5}=\Delta t \sum_{n=1}^{m} \int_{\Omega_{h}}\left(\hat{r}^{n}-\varrho^{n}\right) \frac{p^{\prime}\left(\hat{r}^{n}\right)}{\hat{r}^{n}}\left[\partial_{t} r\right]^{n} \mathrm{~d} x, \\
& S_{6}=-\Delta t \sum_{n=1}^{m} \int_{\Omega_{h}} \frac{\varrho^{n}}{\hat{r}^{n}} p^{\prime}\left(\hat{r}^{n}\right) \boldsymbol{u}^{n} \cdot \nabla r^{n} \mathrm{~d} x,
\end{aligned}
$$

and

$$
\left|G^{m}\right| \leq c \Delta t \sum_{n=1}^{m} \mathcal{E}\left(\varrho^{n}, \hat{\boldsymbol{u}}^{n} \mid \hat{r}^{n}, \hat{\boldsymbol{V}}^{n}\right),\left|R_{h, \Delta t}^{m}\right| \leq c\left(\sqrt{\Delta t}+h^{a}\right),
$$

with the power a defined in (3.9) and with the functional $\mathcal{E}$ introduced in (4.8).

Proof: We take as test functions $\mathbf{U}^{n}=\mathbf{V}_{h, 0}^{n}$ and $r^{n}=\hat{r}^{n}$ in the discrete relative energy inequality (5.1). We keep the left hand side and the first term (term $T_{1}$ ) at the right hand side as they stay. The transformation of the remaining terms at the right hand side (terms $T_{2}-T_{6}$ ) is performed in the following steps:

Step 1: Term $T_{2}$. We have

$$
T_{2}=T_{2,1}+R_{2,1}+R_{2,2}, \text { with } T_{2,1}=\Delta t \sum_{n=1}^{m} \sum_{K \in \mathcal{T}}|K| \varrho_{K}^{n-1} \frac{\boldsymbol{V}_{h, 0, K}^{n}-\boldsymbol{V}_{h, 0, K}^{n-1}}{\Delta t} \cdot\left(\boldsymbol{V}_{h, 0, K}^{n}-\boldsymbol{u}_{K}^{n}\right),
$$

and

$$
R_{2,1}=\Delta t \sum_{n=1}^{m} \sum_{K \in \mathcal{T}} R_{2,1}^{n, K}, \quad R_{2,2}=\Delta t \sum_{n=1}^{m} R_{2,2}^{n}
$$

where

and

$$
R_{2,1}^{n, K}=-\frac{|K|}{2} \varrho_{K}^{n-1} \frac{\left(\boldsymbol{V}_{h, 0, K}^{n}-\boldsymbol{V}_{h, 0, K}^{n-1}\right)^{2}}{\Delta t}=-\frac{|K|}{2} \varrho_{K}^{n-1} \frac{\left(\left[\boldsymbol{V}^{n}-\boldsymbol{V}^{n-1}\right]_{h, 0, K}\right)^{2}}{\Delta t}
$$

$$
R_{2,2}^{n}=-\sum_{K \in \mathcal{T}}|K| \varrho_{K}^{n-1} \frac{\boldsymbol{V}_{h, 0, K}^{n}-\boldsymbol{V}_{h, 0, K}^{n-1}}{\Delta t} \cdot\left(\boldsymbol{u}_{K}^{n-1}-\boldsymbol{u}_{K}^{n}\right) .
$$

We may write by virtue of the first order Taylor formula applied to function $t \mapsto \mathbf{V}(t, x)$,

$$
\begin{gathered}
\left|\frac{\left[\boldsymbol{V}^{n}-\boldsymbol{V}^{n-1}\right]_{h, 0, K}}{\Delta t}\right|=\left|\frac{1}{|K|} \int_{K}\left[\frac{1}{\Delta t}\left[\int_{t_{n-1}}^{t_{n}} \partial_{t} \mathbf{V}(z, x) \mathrm{d} z\right]_{h, 0}\right] \mathrm{d} x\right| \\
=\left|\frac{1}{|K|} \int_{K}\left[\frac{1}{\Delta t} \int_{t_{n-1}}^{t_{n}}\left[\partial_{t} \mathbf{V}(z)\right]_{h, 0}(x) \mathrm{d} z\right] \mathrm{d} x\right| \leq\left\|\left[\partial_{t} \mathbf{V}\right]_{h, 0}\right\|_{L^{\infty}\left(0, T ; L^{\infty}\left(\Omega ; \mathbb{R}^{3}\right)\right)} \leq\left\|\partial_{t} \mathbf{V}\right\|_{L^{\infty}\left(0, T ; L^{\infty}\left(\Omega ; \mathbb{R}^{3}\right)\right)},
\end{gathered}
$$

where we have used the property (2.38) of the projection $\Pi_{h, 0}^{V}$ on the space $V_{h, 0}\left(\Omega_{h}\right)$. Therefore, thanks to the mass conservation (4.1), we get

$$
\left|R_{2,1}^{n, K}\right| \leq \frac{M_{0}}{2}|K| \Delta t\left\|\partial_{t} \boldsymbol{V}\right\|_{L^{\infty}\left(0, T ; L^{\infty}\left(\Omega ; \mathbb{R}^{3}\right)\right)}^{2} .
$$


To treat term $R_{2,2}^{n}$ we use the discrete Hölder inequality and identity (4.1) in order to get

$$
\left|R_{2,2}^{n}\right| \leq \Delta t c M_{0}\left\|\partial_{t} \boldsymbol{V}\right\|_{L^{\infty}\left(0, T ; W^{1, \infty}\left(\Omega ; \mathbb{R}^{3}\right)\right.}^{2}+c M_{0}^{1 / 2}\left(\sum_{K \in \mathcal{T}}|K| \varrho_{K}^{n-1}\left|\boldsymbol{u}_{K}^{n-1}-\boldsymbol{u}_{K}^{n}\right|^{2}\right)^{1 / 2}\left\|\partial_{t} \boldsymbol{V}\right\|_{L^{\infty}\left(0, T ; L^{\infty}\left(\Omega ; \mathbb{R}^{3}\right)\right)}
$$

whence, by virtue of estimate (4.2) for the upwind dissipation term (4.3a), one obtains

$$
\left|R_{2,2}\right| \leq \sqrt{\Delta t} c\left(M_{0}, E_{0},\left\|\partial_{t} \boldsymbol{V}\right\|_{L^{\infty}\left(Q_{T} ; \mathbb{R}^{3}\right)}\right) .
$$

Step 2: Term $T_{3}$. Employing the definition (3.4) of upwind quantities, we easily establish that

$T_{3}=T_{3,1}+R_{3,1}$,

with $T_{3,1}=\Delta t \sum_{n=1}^{m} \sum_{K \in \mathcal{T}} \sum_{\sigma \in \mathcal{E}(K)}|\sigma| \varrho_{\sigma}^{n \text {,up }}\left(\hat{\boldsymbol{u}}_{\sigma}^{n \text {,up }}-\hat{\boldsymbol{V}}_{h, 0, \sigma}^{n, \text { up }}\right) \cdot \boldsymbol{V}_{h, 0, K}^{n} \boldsymbol{u}_{\sigma}^{n} \cdot \boldsymbol{n}_{\sigma, K}, \quad R_{3,1}=\Delta t \sum_{n=1}^{m} \sum_{\sigma \in \mathcal{E}_{\text {int }}} R_{3,1}^{n, \sigma}$,

and $R_{3,1}^{n, \sigma}=|\sigma| \varrho_{K}^{n} \frac{\left|\boldsymbol{V}_{h, 0, K}^{n}-\boldsymbol{V}_{h, 0, L}^{n}\right|^{2}}{2}\left[\boldsymbol{u}_{\sigma}^{n} \cdot \boldsymbol{n}_{\sigma, K}\right]^{+}+|\sigma| \varrho_{L}^{n} \frac{\left|\boldsymbol{V}_{h, 0, L}^{n}-\boldsymbol{V}_{h, 0, K}^{n}\right|^{2}}{2}\left[\boldsymbol{u}_{\sigma}^{n} \cdot \boldsymbol{n}_{\sigma, L}\right]^{+}, \forall \sigma=K \mid L \in \mathcal{E}_{\text {int }}$.

Writing

$$
\begin{aligned}
& \boldsymbol{V}_{h, 0, K}^{n}-\boldsymbol{V}_{h, 0, L}^{n}=\left[\boldsymbol{V}_{h, 0}^{n}-\boldsymbol{V}_{h}^{n}\right]_{K}+\boldsymbol{V}_{h, K}^{n}-\boldsymbol{V}_{h}^{n}+\boldsymbol{V}_{h}^{n}-\boldsymbol{V}_{h, \sigma}^{n} \\
& +\boldsymbol{V}_{h, \sigma}^{n}-\boldsymbol{V}_{h}^{n}+\boldsymbol{V}_{h}^{n}-\boldsymbol{V}_{h, L}^{n}+\left[\boldsymbol{V}_{h}^{n}-\boldsymbol{V}_{h, 0}^{n}\right]_{L}, \sigma=K \mid L \in \mathcal{E}_{\mathrm{int}},
\end{aligned}
$$

and employing estimates (2.47) (if $K \cap \partial \Omega_{h}=\emptyset$ ), (2.48) (if $K \cap \partial \Omega_{h} \neq \emptyset$ ) to evaluate the $L^{\infty}$-norm of the first term, (2.51) then $(2.40) s_{s=1}$ and (2.52) after (2.40) $s=1$ to evaluate the $L^{\infty}$-norm of the second and third terms, and performing the same tasks at the second line, we get

$$
\left\|\boldsymbol{V}_{h, 0, K}^{n}-\boldsymbol{V}_{h, 0, L}^{n}\right\|_{L^{\infty}\left(K \cup L ; \mathbb{R}^{3}\right)} \leq \operatorname{ch\| \nabla \boldsymbol {V}\| _{L^{\infty }(K\cup L;\mathbb {R}^{9})}}
$$

consequently

$$
\left|R_{3,1}^{n, \sigma}\right| \leq h^{2} c\|\nabla \boldsymbol{V}\|_{L^{\infty}\left((0, T) \times \Omega ; \mathbb{R}^{9}\right)}^{2}|\sigma|\left(\varrho_{K}^{n}+\varrho_{L}^{n}\right)\left|\boldsymbol{u}_{\sigma}^{n}\right|, \forall \sigma=K \mid L \in \mathcal{E}_{\text {int }},
$$

whence

$$
\begin{aligned}
\left|R_{3,1}\right| & \leq h c\|\nabla \boldsymbol{V}\|_{L^{\infty}\left((0, T) \times \Omega ; \mathbb{R}^{9}\right)}^{2}\left(\sum_{K \in \mathcal{T}} \sum_{\sigma=K \mid L \in \mathcal{E}(K)} h|\sigma|\left(\varrho_{K}^{n}+\varrho_{L}^{n}\right)^{6 / 5}\right)^{5 / 6} \times \\
& {\left[\Delta t \sum_{n=1}^{m}\left(\sum_{K \in \mathcal{T}} \sum_{\sigma \in \mathcal{E}(K)} h\left|\sigma \| \boldsymbol{u}_{\sigma}^{n}\right|^{6}\right)^{1 / 3}\right]^{1 / 2} \leq h c\left(M_{0}, E_{0},\|\nabla \boldsymbol{V}\|_{L^{\infty}\left(Q_{T} ; \mathbb{R}^{9}\right)}\right), }
\end{aligned}
$$

provided $\gamma \geq 6 / 5$, thanks to the discrete Hölder inequality, the equivalence relation (2.29), the equivalence of norms (2.41) and energy bounds listed in Corollary 4.1.

Clearly, for each face $\sigma=K \mid L \in \mathcal{E}_{\text {int }}, \boldsymbol{u}_{\sigma}^{n} \cdot \boldsymbol{n}_{\sigma, K}+\boldsymbol{u}_{\sigma}^{n} \cdot \mathbf{n}_{\sigma, L}=0$; whence, finally

$$
T_{3,1}=\Delta t \sum_{n=1}^{m} \sum_{K \in \mathcal{T}} \sum_{\sigma \in \mathcal{E}(K)}|\sigma| \varrho_{\sigma}^{n, \text { up }}\left(\hat{\boldsymbol{u}}_{\sigma}^{n, \text { up }}-\hat{\boldsymbol{V}}_{h, 0, \sigma}^{n, \text { up }}\right) \cdot\left(\boldsymbol{V}_{h, 0, K}^{n}-\boldsymbol{V}_{h, 0, \sigma}^{n}\right) \boldsymbol{u}_{\sigma}^{n} \cdot \boldsymbol{n}_{\sigma, K} .
$$

Before the next transformation of term $T_{3,1}$, we realize that

$$
\mathbf{V}_{h, 0, K}^{n}-\mathbf{V}_{h, 0, \sigma}^{n}=\left[\mathbf{V}_{h, 0}^{n}-\mathbf{V}_{h}^{n}\right]_{K}+\mathbf{V}_{h, K}^{n}-\mathbf{V}_{h}^{n}+\mathbf{V}_{h}^{n}-\mathbf{V}_{h, \sigma}^{n}+\left[\mathbf{V}_{h}^{n}-\mathbf{V}_{h, 0}^{n}\right]_{\sigma}
$$

whence by virtue of $(2.47 \sqrt{2.48}),(2.51,2.52)$ and $(2.40) s=1$, similarly as in (6.7),

$$
\left\|\boldsymbol{V}_{h, 0, K}^{n}-\boldsymbol{V}_{h, 0, \sigma}^{n}\right\|_{L^{\infty}\left(K ; \mathbb{R}^{3}\right)} \leq \operatorname{ch}\left\|\nabla_{x} \boldsymbol{V}\right\|_{L^{\infty}\left(0, T ; L^{\infty}\left(\Omega: R^{3}\right)\right)}, \quad \sigma \subset K .
$$


Let us now decompose the term $T_{3,1}$ as

$$
\begin{aligned}
& T_{3,1}=T_{3,2}+R_{3,2}, \text { with } R_{3,2}=\Delta t \sum_{n=1}^{m} R_{3,2}^{n}, \\
& T_{3,2}=\Delta t \sum_{n=1}^{m} \sum_{K \in \mathcal{T}} \sum_{\sigma \in \mathcal{E}(K)}|\sigma| \varrho_{\sigma}^{n, \mathrm{up}}\left(\hat{\boldsymbol{V}}_{h, 0, \sigma}^{n, \mathrm{up}}-\hat{\boldsymbol{u}}_{\sigma}^{n, \mathrm{up}}\right) \cdot\left(\boldsymbol{V}_{h, 0, \sigma}^{n}-\boldsymbol{V}_{h, 0, K}^{n}\right) \hat{\boldsymbol{u}}_{\sigma}^{n, \mathrm{up}} \cdot \boldsymbol{n}_{\sigma, K}, \text { and } \\
& R_{3,2}^{n}=\sum_{K \in \mathcal{T}} \sum_{\sigma \in \mathcal{E}(K)}|\sigma| \varrho_{\sigma}^{n, \mathrm{up}}\left(\hat{\boldsymbol{V}}_{h, 0, \sigma}^{n, \mathrm{up}}-\hat{\boldsymbol{u}}_{\sigma}^{n, \mathrm{up}}\right) \cdot\left(\boldsymbol{V}_{h, 0, \sigma}^{n}-\boldsymbol{V}_{h, 0, K}^{n}\right)\left(\boldsymbol{u}_{\sigma}^{n}-\hat{\boldsymbol{u}}_{\sigma}^{n, \mathrm{up}}\right) \cdot \boldsymbol{n}_{\sigma, K} .
\end{aligned}
$$

By virtue of discrete Hölder's inequality and estimate (6.10), we get

$$
\begin{aligned}
\left|R_{3,2}^{n}\right| & \leq c\|\nabla \mathbf{V}\|_{L^{\infty}\left(Q_{T} ; \mathbb{R}^{9}\right)}\left(\sum_{K \in \mathcal{T}} \sum_{\sigma \in \mathcal{E}(K)} h|\sigma| \varrho_{\sigma}^{n, \text { up }}\left|\hat{\boldsymbol{u}}_{\sigma}^{n, \text { up }}-\hat{\boldsymbol{V}}_{h, 0, \sigma}^{n, \text { up }}\right|^{2}\right)^{1 / 2} \\
& \times\left(\sum_{K \in \mathcal{T}} \sum_{\sigma \in \mathcal{E}(K)} h\left|\sigma \| \varrho_{\sigma}^{n, \text { up }}\right|^{\gamma_{0}}\right)^{1 /\left(2 \gamma_{0}\right)}\left(\sum_{K \in \mathcal{T}} \sum_{\sigma \in \mathcal{E}(K)} h|\sigma|\left|\boldsymbol{u}_{\sigma}^{n}-\hat{\boldsymbol{u}}_{\sigma}^{n, \mathrm{up}}\right|^{q}\right)^{1 / q},
\end{aligned}
$$

where $\frac{1}{2}+\frac{1}{2 \gamma_{0}}+\frac{1}{q}=1, \gamma_{0}=\min \{\gamma, 2\}$ and $\gamma \geq 3 / 2$. For the sum in the last term of the above product, we have

$$
\begin{gathered}
\sum_{K \in \mathcal{T}} \sum_{\sigma \in \mathcal{E}(K)} h|\sigma|\left|\boldsymbol{u}_{\sigma}^{n}-\hat{\boldsymbol{u}}_{\sigma}^{n, \text { up }}\right|^{q} \leq c \sum_{K \in \mathcal{T}} \sum_{\sigma \in \mathcal{E}(K)} h\left|\sigma \| \boldsymbol{u}_{\sigma}^{n}-\boldsymbol{u}_{K}^{n}\right|^{q} \\
\leq c\left(\sum_{K \in \mathcal{T}} \sum_{\sigma \in \mathcal{E}(K)}\left(\left\|\boldsymbol{u}_{\sigma}^{n}-\boldsymbol{u}^{n}\right\|_{L^{q}\left(K ; \mathbb{R}^{3}\right)}^{q}+\sum_{K \in \mathcal{T}}\left\|\boldsymbol{u}^{n}-\boldsymbol{u}_{K}^{n}\right\|_{L^{q}\left(K ; \mathbb{R}^{3}\right)}^{q}\right) \leq c h^{\frac{2 \gamma_{0}-3}{2 \gamma_{0}} q}\left(\sum_{K \in \mathcal{T}}\left\|\nabla_{x} \boldsymbol{u}^{n}\right\|_{L^{2}\left(K ; \mathbb{R}^{9}\right.}^{2}\right)^{q / 2},\right.
\end{gathered}
$$

where we have used the definition (3.4), the discrete Minkowski inequality, interpolation inequalities (2.55 2.56) and the discrete 'imbedding' inequality (2.57). Now we can go back to the estimate of $R_{3,2}^{n}$ taking into account the upper bounds (4.4), (4.74.8), in order to get

$$
\left|R_{3,2}\right| \leq h^{a} c\left(M_{0}, E_{0},\|\nabla \mathbf{V}\|_{L^{\infty}\left(Q_{T} ; \mathbb{R}^{9}\right)}\right),
$$

provided $\gamma \geq 3 / 2$, where $a$ is given in (6.3).

Finally, we rewrite term $T_{3,2}$ as

$$
\begin{aligned}
& T_{3,2}=T_{3,3}+R_{3,3}, \text { with } R_{3,3}=\Delta t \sum_{n=1}^{m} R_{3,3}^{n}, \\
& T_{3,3}=\Delta t \sum_{n=1}^{m} \sum_{K \in \mathcal{T}} \sum_{\sigma \in \mathcal{E}(K)}|\sigma| \varrho_{\sigma}^{n, \text { up }}\left(\hat{\boldsymbol{V}}_{h, 0, \sigma}^{n, \text { up }}-\hat{\boldsymbol{u}}_{\sigma}^{n, \text { up }}\right) \cdot\left(\boldsymbol{V}_{h, 0, \sigma}^{n}-\boldsymbol{V}_{h, 0, K}^{n}\right) \hat{\boldsymbol{V}}_{h, 0, \sigma}^{n, \text { up }} \cdot \boldsymbol{n}_{\sigma, K}, \text { and } \\
& R_{3,3}^{n}=\sum_{K \in \mathcal{T}} \sum_{\sigma \in \mathcal{E}(K)}|\sigma| \varrho_{\sigma}^{n, \text { up }}\left(\hat{\boldsymbol{V}}_{h, 0, \sigma}^{n \text {,up }}-\hat{\boldsymbol{u}}_{\sigma}^{n, \text { up }}\right) \cdot\left(\boldsymbol{V}_{h, 0, \sigma}^{n}-\boldsymbol{V}_{h, 0, K}^{n}\right)\left(\hat{\boldsymbol{u}}_{\sigma}^{n, \text { up }}-\hat{\boldsymbol{V}}_{h, 0, \sigma}^{n, \text { up }}\right) \cdot \boldsymbol{n}_{\sigma, K} ;
\end{aligned}
$$

whence

$$
\left|R_{3,3}\right| \leq c\left(\|\nabla \boldsymbol{V}\|_{L^{\infty}\left(Q_{T}, \mathbb{R}^{9}\right)}\right) \Delta t \sum_{n=1}^{m} \mathcal{E}\left(\varrho^{n}, \hat{\mathbf{u}}^{n} \mid \hat{r}^{n}, \hat{\boldsymbol{V}}_{h, 0}^{n}\right) .
$$

Step 3: $\operatorname{Term} T_{4}$. Integration by parts over each $K \in \mathcal{T}$ gives

$$
T_{4}=-\Delta t \sum_{n=1}^{m} \sum_{K \in \mathcal{T}} \int_{\mathrm{K}} p\left(\varrho_{K}^{n}\right) \operatorname{div}_{x} \boldsymbol{V}_{h, 0}^{n} \mathrm{~d} x
$$

We may write

$$
\left\|\operatorname{div}_{x}\left(\boldsymbol{V}_{0, h}^{n}-\boldsymbol{V}_{h}^{n}\right)\right\|_{L^{\infty}(K)} \leq \operatorname{ch}\left\|\nabla_{x} \boldsymbol{V}\right\|_{L^{\infty}\left(0, T ; L^{\infty}\left(\Omega ; \mathbb{R}^{9}\right)\right)},
$$


where we have used (2.47 2.48). Therefore, employing identity (2.43) we obtain

$$
\begin{gathered}
T_{4}=T_{4,1}+R_{4,1}, \quad T_{4,1}=-\Delta t \sum_{n=1}^{m} \sum_{K \in \mathcal{T}} \int_{\mathrm{K}} p\left(\varrho_{K}^{n}\right) \operatorname{div}_{x} \boldsymbol{V}^{n} \mathrm{~d} x, \\
R_{4,1}=-\Delta t \sum_{n=1}^{m} \sum_{K \in \mathcal{T}} \int_{\mathrm{K}} p\left(\varrho_{K}^{n}\right) \operatorname{div}_{x}\left(\boldsymbol{V}_{h, 0}^{n}-\boldsymbol{V}_{h}^{n}\right) \mathrm{d} x .
\end{gathered}
$$

Due to (1.4) and (4.7), $p\left(\varrho^{n}\right)$ is bounded uniformly in $L^{\infty}\left(L^{1}(\Omega)\right)$; employing this fact and (6.14) we immediately get

$$
\left|R_{4,1}\right| \leq h c\left(E_{0}, M_{0},\|\nabla \mathbf{V}\|_{L^{\infty}\left(0, T ; L^{\infty}\left(\Omega ; \mathbb{R}^{9}\right)\right)}\right) .
$$

Step 4: Term $T_{5}$. Using the Taylor formula, we get

$$
H^{\prime}\left(r_{K}^{n}\right)-H^{\prime}\left(r_{K}^{n-1}\right)=H^{\prime \prime}\left(r_{K}^{n}\right)\left(r_{K}^{n}-r_{K}^{n-1}\right)-\frac{1}{2} H^{\prime \prime \prime}\left(\bar{r}_{K}^{n}\right)\left(r_{K}^{n}-r_{K}^{n-1}\right)^{2},
$$

where $\bar{r}_{K}^{n} \in\left[\min \left(r_{K}^{n-1}, r_{K}^{n}\right), \max \left(r_{K}^{n-1}, r_{K}^{n}\right)\right] ;$ we infer

$$
\begin{aligned}
& T_{5}=T_{5,1}+R_{5,1}, \text { with } T_{5,1}=\Delta t \sum_{n=1}^{m} \sum_{K \in \mathcal{T}}|K|\left(r_{K}^{n}-\varrho_{K}^{n}\right) \frac{p^{\prime}\left(r_{K}^{n}\right)}{r_{K}^{n}} \frac{r_{K}^{n}-r_{K}^{n-1}}{\Delta t}, R_{5,1}=\Delta t \sum_{n=1}^{m} \sum_{K \in \mathcal{T}} R_{5,1}^{n, K}, \text { and } \\
& R_{5,1}^{n, K}=\frac{1}{2}|K| H^{\prime \prime \prime}\left(\bar{r}_{K}^{n}\right) \frac{\left(r_{K}^{n}-r_{K}^{n-1}\right)^{2}}{\Delta t}\left(\varrho_{K}^{n}-r_{K}^{n}\right) .
\end{aligned}
$$

Consequently, by the first order Taylor formula applied to function $t \mapsto r(t, x)$ on the interval $\left(t_{n-1}, t_{n}\right)$ and thanks to the mass conservation (4.1)

$$
\left|R_{5,1}\right| \leq \Delta t c\left(M_{0}, \underline{r}, \bar{r},\left|p^{\prime}\right|_{C^{1}([\underline{r}, \bar{r}]},\left\|\partial_{t} r\right\|_{L^{\infty}\left(Q_{T}\right)}\right) .
$$

Let us now decompose $T_{5,1}$ as follows:

$$
\begin{aligned}
& T_{5,1}=T_{5,2}+R_{5,2}, \text { with } T_{5,2}=\Delta t \sum_{n=1}^{m} \sum_{K \in \mathcal{T}} \int_{K}\left(r_{K}^{n}-\varrho_{K}^{n}\right) \frac{p^{\prime}\left(r_{K}^{n}\right)}{r_{K}^{n}}\left[\partial_{t} r\right]^{n} \mathrm{~d} x, R_{5,2}=\Delta t \sum_{n=1}^{m} \sum_{K \in \mathcal{T}} R_{5,2}^{n, K}, \text { and } \\
& R_{5,2}^{n, K}=\int_{K}\left(r_{K}^{n}-\varrho_{K}^{n}\right) \frac{p^{\prime}\left(r_{K}^{n}\right)}{r_{K}^{n}}\left(\frac{r_{K}^{n}-r_{K}^{n-1}}{\Delta t}-\left[\partial_{t} r\right]^{n}\right) \mathrm{d} x .
\end{aligned}
$$

In accordance with (3.2), here and in the sequel, $\left[\partial_{t} r\right]^{n}(x)=\partial_{t} r\left(t_{n}, x\right)$. We write using twice the Taylor formula in the integral form and the Fubini theorem,

$$
\begin{aligned}
\left|R_{5,2}^{n, K}\right| & =\frac{1}{\Delta t}\left|p^{\prime}\left(r_{K}^{n}\right) r_{K}^{n}\left(\varrho_{K}^{n}-r_{K}^{n}\right) \int_{K} \int_{t_{n-1}}^{t_{n}} \int_{s}^{t_{n}} \partial_{t}^{2} r(z) \mathrm{d} z \mathrm{~d} s \mathrm{~d} x\right| \\
& \leq \frac{p^{\prime}\left(r_{K}^{n}\right)}{r_{K}^{n}} \int_{t_{n-1}}^{t_{n}} \int_{K}\left|\varrho_{K}^{n}-r_{K}^{n}\right|\left|\partial_{t}^{2} r(z)\right| \mathrm{d} x \mathrm{~d} z \mathrm{~d} s \\
\leq & \frac{p^{\prime}\left(r_{K}^{n}\right)}{r_{K}^{n}}\left\|\varrho^{n}-\hat{r}^{n}\right\|_{L^{\gamma}(K)} \int_{t_{n-1}}^{t_{n}}\left\|\partial_{t}^{2} r(z)\right\|_{L^{\gamma^{\prime}}(K)} \mathrm{d} z \mathrm{~d} s .
\end{aligned}
$$

Therefore, by virtue of Corollary 4.1, we have estimate

$$
\left|R_{5,2}\right| \leq \Delta t c\left(M_{0}, E_{0}, \underline{r}, \bar{r},\left|p^{\prime}\right|_{C^{1}([\underline{r}, \bar{r}]},\left\|\partial_{t}^{2} r\right\|_{L^{1}\left(0, T ; L^{\gamma^{\prime}}(\Omega)\right.}\right) .
$$


Step 5: Term $T_{6}$. We decompose this term as follows:

$$
\begin{aligned}
& T_{6}=T_{6,1}+R_{6,1}, \quad R_{6,1}=\Delta t \sum_{n=1}^{m} \sum_{K \in \mathcal{T}} \sum_{\sigma \in \mathcal{E}(K)} R_{6,1}^{n, \sigma, K}, \text { with } \\
& T_{6,1}=\Delta t \sum_{n=1}^{m} \sum_{K \in \mathcal{T}} \sum_{\sigma=K \mid L \in \mathcal{E}(K)}|\sigma| \varrho_{K}^{n}\left(H^{\prime}\left(r_{K}^{n-1}\right)-H^{\prime}\left(r_{\sigma}^{n-1}\right)\right) \boldsymbol{u}_{\sigma}^{n} \cdot \boldsymbol{n}_{\sigma, K}, \text { and } \\
& R_{6,1}^{n, \sigma, K}=|\sigma|\left(\varrho_{\sigma}^{n, \mathrm{up}}-\varrho_{K}^{n}\right)\left(H^{\prime}\left(r_{K}^{n-1}\right)-H^{\prime}\left(r_{\sigma}^{n-1}\right)\right) \boldsymbol{u}_{\sigma}^{n} \cdot \boldsymbol{n}_{\sigma, K}, \text { for } \sigma=K \mid L \in \mathcal{E}_{\text {int }} .
\end{aligned}
$$

We will now estimate the term $R_{6,1}^{n, \sigma, K}$. We shall treat separately the cases $\gamma<2$ and $\gamma \geq 2$. The 'simple' case $\gamma \geq 2$ is left to the reader. The more complicated case $\gamma<2$ will be treated as follows: We first write

$$
\begin{gathered}
\left|R_{6,1}^{n, \sigma, K}\right| \leq \sqrt{h}\left\|\nabla H^{\prime}(r)\right\|_{L^{\infty}\left(Q_{T} ; \mathbb{R}^{3}\right)}|\sigma|\left|\varrho_{\sigma}^{n, \text { up }}-\varrho_{K}^{n}\right|\left[\frac{1_{\left\{\varrho_{\sigma}^{n} \geq 1\right\}}}{\left[\max \left\{\varrho_{K}, \varrho_{L}\right\}\right]^{(2-\gamma) / 2}}+1_{\left\{\varrho_{\sigma}^{n}<1\right\}}\right] \sqrt{\left|\boldsymbol{u}_{\sigma}^{n} \cdot \boldsymbol{n}_{\sigma, K}\right|} \times \\
{\left[1_{\left\{\varrho_{\sigma}^{n} \geq 1\right\}}\left[\max \left\{\varrho_{K}, \varrho_{L}\right\}\right]^{(2-\gamma) / 2}+1_{\left\{\varrho_{\sigma}^{n}<1\right\}}\right] \sqrt{h} \sqrt{\left|\boldsymbol{u}_{\sigma}^{n} \cdot \boldsymbol{n}_{\sigma, K}\right|},}
\end{gathered}
$$

where we have employed the first order Taylor formula applied to function $x \mapsto H^{\prime}\left(r\left(t_{n-1}, x\right)\right.$. Consequently, the application of the discrete Hölder and Young inequalities yield

$$
\begin{aligned}
\left|R_{6,1}\right| & \leq \sqrt{h} c\left\|\nabla H^{\prime}(r)\right\|_{L^{\infty}\left(Q_{T} ; \mathbb{R}^{3}\right)^{\times}} \\
& \Delta t \sum_{n=1}^{m}\left(\sum_{K \in \mathcal{T}} \sum_{\sigma \in \mathcal{E}(K)}|\sigma| h\left[1_{\left\{\varrho_{\sigma}^{n} \geq 1\right\}}\left[\max \left\{\varrho_{K}, \varrho_{L}\right\}\right]^{2-\gamma}+1_{\left\{\bar{\varrho}_{\sigma}^{n}<1\right\}}\right]\left|\boldsymbol{u}_{\sigma}^{n} \cdot \boldsymbol{n}_{\sigma, K}\right|\right)^{1 / 2} \times \\
& \left(\sum_{K \in \mathcal{T}} \sum_{\sigma=K \mid L \in \mathcal{E}(K)}|\sigma| h\left(\varrho_{\sigma}^{n, \text { up }}-\varrho_{K}^{n}\right)^{2}\left[\frac{1_{\left\{\bar{\varrho}_{\sigma}^{n} \geq 1\right\}}}{\left[\max \left\{\varrho_{K}, \varrho_{L}\right\}\right]^{2-\gamma}}+1_{\left\{\varrho_{\sigma}^{n}<1\right\}}\right]\left|\boldsymbol{u}_{\sigma}^{n} \cdot \boldsymbol{n}_{\sigma, K}\right|\right)^{1 / 2} \\
& \leq \sqrt{h} c\left\|\nabla H^{\prime}(r)\right\|_{L^{\infty}\left(Q_{T} ; \mathbb{R}^{3}\right)} \times \\
& \Delta t \sum_{n=1}^{m}\left\{\left[\left|\Omega_{h}\right|^{\frac{5}{6}}+\left(\sum_{K \in \mathcal{T}}|\sigma| h\left(\varrho_{K}^{n}\right)^{\frac{6}{5}(2-\gamma)}\right)^{\frac{5}{6}}\right]\left(\sum_{K \in \mathcal{T}} \sum_{\sigma \in \mathcal{E}(K)}|\sigma| h\left|\boldsymbol{u}_{\sigma}^{n} \cdot \boldsymbol{n}_{\sigma, K}\right|^{6}\right)^{\frac{1}{6}}\right. \\
& \left.\sum_{K \in \mathcal{T} \sigma=K \mid L \in \mathcal{E}(K)}|\sigma| h\left(\varrho_{\sigma}^{n, \text { up }}-\varrho_{K}^{n}\right)^{2}\left[\frac{1_{\left\{\varrho_{\sigma}^{n} \geq 1\right\}}}{\left[\max \left\{\varrho_{K}, \varrho_{L}\right\}\right]^{2-\gamma}}+1_{\left\{\varrho_{\sigma}^{n}<1\right\}}\right] \mid \boldsymbol{u}_{\sigma}^{n} \cdot \boldsymbol{n}_{\sigma, K}\right\}^{1 / 2} \\
& \leq \sqrt{h} c\left\|\nabla H^{\prime}(r)\right\|_{L^{\infty}\left(Q_{T} ; \mathbb{R}^{3}\right)}\left\{\Delta t \sum_{n=1}^{m}\left[\left|\Omega_{h}\right|^{\frac{5}{6}}+\left(\sum_{K \in \mathcal{T}}|\sigma| h\left(\varrho_{K}^{n}\right)^{\frac{6}{5}(2-\gamma)}\right)^{\frac{5}{6}}\right]\left(\sum_{\sigma \in \mathcal{E}}|\sigma| h\left|\boldsymbol{u}_{\sigma}^{n}\right|^{6}\right)^{1 / 6}\right. \\
& \left.+\Delta t \sum_{n=1}^{m}\left[\sum_{K \in \mathcal{T}} \sum_{\sigma=K \mid L \in \mathcal{E}(K)}|\sigma| h\left(\varrho_{\sigma}^{n, \text { up }}-\varrho_{K}^{n}\right)^{2}\left[\frac{1_{\left\{\bar{\varrho}_{\sigma}^{n} \geq 1\right\}}}{\left[\max \left\{\varrho_{K}, \varrho_{L}\right\}\right]^{2-\gamma}}+1_{\left\{\bar{\varrho}_{\sigma}^{n}<1\right\}}\right]\left|\boldsymbol{u}_{\sigma}^{n} \cdot \boldsymbol{n}_{\sigma, K}\right|\right]\right\} \\
& \leq \sqrt{h} c\left(M_{0}, E_{0}, \underline{r}, \bar{r},\left|p^{\prime}\right|_{C([\underline{r}, \bar{r}])},\|\nabla r\|_{L^{\infty}\left(Q_{T} ; \mathbb{R}^{3}\right)}\right),
\end{aligned}
$$

where, in order to get the last line, we have used the estimate (4.9) of the numerical dissipation to evaluate the second term, and finally equivalence of norms (2.41) $p=6$ together with (4.5) and (4.7), under assumption $\gamma \geq 12 / 11$, to evaluate the first term.

Let us now decompose the term $T_{6,1}$ as

$$
\begin{aligned}
& T_{6,1}=T_{6,2}+R_{6,2}, \text { with } T_{6,2}=\Delta t \sum_{n=1}^{m} \sum_{K \in \mathcal{T}} \sum_{\sigma=K \mid L \in \mathcal{E}(K)}|\sigma| \varrho_{K}^{n} H^{\prime \prime}\left(r_{K}^{n-1}\right)\left(r_{K}^{n-1}-r_{\sigma}^{n-1}\right)\left[\boldsymbol{u}_{\sigma}^{n} \cdot \boldsymbol{n}_{\sigma, K}\right], \\
& R_{6,2}=\Delta t \sum_{n=1}^{m} \sum_{K \in \mathcal{K}} \sum_{\sigma \in \mathcal{E}(K)} R_{6,2}^{n, \sigma, K}, \text { and } \\
& R_{6,2}^{n, \sigma, K}=|\sigma| \varrho_{K}^{n}\left(H^{\prime}\left(r_{K}^{n-1}\right)-H^{\prime}\left(r_{\sigma}^{n-1}\right)-H^{\prime \prime}\left(r_{K}^{n-1}\right)\left(r_{K}^{n-1}-r_{\sigma}^{n-1}\right)\right)\left[\boldsymbol{u}_{\sigma}^{n} \cdot \boldsymbol{n}_{\sigma, K}\right] .
\end{aligned}
$$


Therefore, by virtue of the second order Taylor formula applied to function $H^{\prime}$, the Hölder inequality, (2.41), and (4.5), (4.7) in Corollary 4.1, we have, provided $\gamma \geq 6 / 5$,

$$
\begin{aligned}
\left|R_{6,2}\right| & \leq h c\left(\left|H^{\prime \prime}\right|_{C([\underline{r}, \bar{r}])}+\left|H^{\prime \prime \prime}\right|_{C([\underline{r}, \bar{r}])}\right)\|\nabla r\|_{L^{\infty}\left(Q_{T} ; \mathbb{R}^{3}\right)} \Delta t \sum_{n=1}^{m}\left\|\varrho^{n}\right\|_{L^{\gamma}\left(\Omega_{h}\right)}\left\|\boldsymbol{u}^{n}\right\|_{L^{6}\left(\Omega_{h} ; \mathbb{R}^{3}\right)} \\
& \leq h c\left(M_{0}, E_{0}, \underline{r}, \bar{r},\left|p^{\prime}\right|_{C^{1}([\underline{r}, \bar{r}])},\|\nabla r\|_{L^{\infty}\left(Q_{T} ; \mathbb{R}^{3}\right)}\right) .
\end{aligned}
$$

Let us now deal with the term $T_{6,2}$. Noting that $\int_{K} \nabla r^{n-1} \mathrm{~d} x=\sum_{\sigma \in \mathcal{E}(K)}|\sigma|\left(r_{\sigma}^{n-1}-r_{K}^{n-1}\right) \boldsymbol{n}_{\sigma, K}$, we may write $T_{6,2}=T_{6,3}+R_{6,3}$, with

$$
\begin{aligned}
& T_{6,3}=-\Delta t \sum_{n=1}^{m} \sum_{K \in \mathcal{T}} \int_{K} \varrho_{K}^{n} H^{\prime \prime}\left(r_{K}^{n-1}\right) \boldsymbol{u}^{n} \cdot \nabla r^{n-1} \mathrm{~d} x, \\
& R_{6,3}=\Delta t \sum_{n=1}^{m} \sum_{K \in \mathcal{T}} \int_{K} \varrho_{K}^{n} H^{\prime \prime}\left(r_{K}^{n-1}\right)\left(\boldsymbol{u}^{n}-\boldsymbol{u}_{K}^{n}\right) \cdot \nabla r^{n-1} \mathrm{~d} x \\
& \quad+\Delta t \sum_{n=1}^{m} \sum_{K \in \mathcal{T}} \sum_{\sigma \in \mathcal{E}(K)}|\sigma| \varrho_{K}^{n} H^{\prime \prime}\left(r_{K}^{n-1}\right)\left(r_{K}^{n-1}-r_{\sigma}^{n-1}\right)\left(\boldsymbol{u}_{\sigma}^{n}-\boldsymbol{u}_{K}^{n}\right) \cdot \boldsymbol{n}_{\sigma, K} .
\end{aligned}
$$

Consequently, by virtue of Hölder's inequality, interpolation inequality (2.55) (to estimate $\left\|\mathbf{u}^{n}-\mathbf{u}_{K}^{n}\right\|_{L^{\gamma_{0}^{\prime}}\left(K ; \mathbb{R}^{3}\right)}$ by $\left.h^{\left(5 \gamma_{0}-6\right) /\left(2 \gamma_{0}\right)}\left\|\nabla_{x} \mathbf{u}^{n}\right\|_{L^{2}\left(K ; \mathbb{R}^{9}\right)}, \gamma_{0}=\min \{\gamma, 2\}\right)$ in the first term, and by the Taylor formula applied to

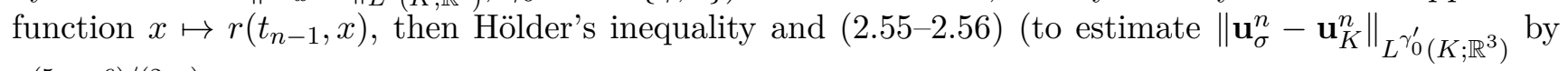
$\left.h^{\left(5 \gamma_{0}-6\right) /\left(2 \gamma_{0}\right)}\left\|\nabla_{x} \mathbf{u}^{n}\right\|_{L^{2}\left(K ; \mathbb{R}^{9}\right)}\right)$, we get

$$
\left|R_{6,3}\right| \leq h^{b} c\left(M_{0}, E_{0}, \underline{r}, \bar{r},\left|p^{\prime}\right|_{C^{1}([\underline{r}, \bar{r}])}\|\nabla r\|_{L^{\infty}\left(Q_{T} ; \mathbb{R}^{3}\right)}\right), \quad b=\frac{5 \gamma_{0}-6}{2 \gamma_{0}},
$$

provided $\gamma \geq 6 / 5$, where we have used at the end the discrete imbedding and Hölder inequalities (2.572.58) and finally estimates (4.4) and (4.7).

Finally we write $T_{6,3}=T_{6,4}+R_{6,4}$, with

$$
\begin{aligned}
& T_{6,4}=-\Delta t \sum_{n=1}^{m} \sum_{K \in \mathcal{T}} \int_{K} \varrho_{K}^{n} \frac{p^{\prime}\left(r_{K}^{n}\right)}{r_{K}^{n}} \boldsymbol{u}^{n} \cdot \nabla r^{n} \mathrm{~d} x \\
& R_{6,4}=\Delta t \sum_{n=1}^{m} \sum_{K \in \mathcal{T}} \int_{K} \varrho_{K}^{n}\left(H^{\prime \prime}\left(r_{K}^{n}\right) \nabla r^{n}-H^{\prime \prime}\left(r_{K}^{n-1}\right) \nabla r^{n-1}\right) \cdot \boldsymbol{u}^{n} \mathrm{~d} x
\end{aligned}
$$

where by the same token as in (6.19),

$$
\left|R_{6,4}\right| \leq \Delta t c\left(M_{0}, E_{0}, \underline{r}, \bar{r},\left|p^{\prime}\right|_{C^{1}([\underline{r}, \bar{r}])},\left\|\nabla r, \partial_{t} r\right\|_{L^{\infty}\left(Q_{T} ; \mathbb{R}^{4}\right)},\left\|\partial_{t} \nabla r\right\|_{L^{2}\left(0, T ; L^{\left.6 \gamma /(5 \gamma-6)\left(\Omega ; \mathbb{R}^{3}\right)\right)}\right)}\right)
$$

provided $\gamma \geq 6 / 5$.

We are now in position to conclude the proof of Lemma 6.1; we obtain the inequality (6.1) by gathering the principal terms (6.4), (6.12), (6.15), (6.18), (6.22) and the residual terms estimated in (6.5), (6.6), (6.8), (6.11), (6.13), (6.17), (6.19), (6.20), (6.21), (6.23) at the right hand side $\sum_{i=1}^{6} T_{i}$ of the discrete relative energy inequality (5.1). 


\section{$7 \quad$ A discrete identity satisfied by the strong solution}

This section is devoted to the proof of a discrete identity satisfied by any strong solution of problem (1.11.6) in the class (2.9 2.10) extended eventually to $\mathbb{R}^{3}$ according to Lemma 2.1. This identity is stated in Lemma 7.1 below. It will be used in combination with the approximate relative energy inequality stated in Lemma 6.1 to deduce the convenient form of the relative energy inequality verified by any function being a strong solution to the compressible Navier-Stokes system. This last step is performed in the next section.

Lemma 7.1 (A discrete identity for strong solutions). Let $\left(\varrho^{n}, \boldsymbol{u}^{n}\right)$ be a solution of the discrete problem (3.5 3.7) with the pressure satisfying (1.4), where $\gamma \geq 3 / 2$. There exists

$$
\begin{gathered}
c=c\left(M_{0}, E_{0}, \underline{r}, \bar{r},\left|p^{\prime}\right|_{C^{1}[\underline{r}, \bar{r}]},\left\|\left(\partial_{t} r, \nabla r, \boldsymbol{V}, \partial_{t} \boldsymbol{V}, \nabla \boldsymbol{V}, \nabla^{2} \boldsymbol{V}\right)\right\|_{L^{\infty}\left(Q_{T} ; \mathbb{R}^{45}\right)},\right. \\
\left.\left\|\partial_{t}^{2} r\right\|_{L^{1}\left(0, T ; L^{\prime}(\Omega)\right)},\left\|\partial_{t} \nabla r\right\|_{L^{2}\left(0, T ; L^{6 \gamma / 5 \gamma-6}\left(\Omega ; \mathbb{R}^{3}\right)\right)},\left\|\partial_{t}^{2} \boldsymbol{V}, \partial_{t} \nabla \boldsymbol{V}\right\|_{L^{2}\left(0, T ; L^{6 / 5}\left(\Omega ; \mathbb{R}^{12}\right)\right)}\right)>0,
\end{gathered}
$$

such that for all $m=1, \ldots, N$, we have:

$$
\sum_{i=1}^{6} \mathcal{S}_{i}+\mathcal{R}_{h, \Delta t}^{m}=0
$$

where

$$
\begin{aligned}
& \mathcal{S}_{1}=\Delta t \sum_{n=1}^{m} \sum_{K \in \mathcal{T}}\left(\mu \int_{K} \nabla_{x} \boldsymbol{V}_{h, 0}^{n}: \nabla_{x}\left(\boldsymbol{V}_{h, 0}^{n}-\boldsymbol{u}^{n}\right) \mathrm{d} x+\frac{\mu}{3} \int_{K} \operatorname{div} \boldsymbol{V}_{h, 0}^{n} \operatorname{div}\left(\boldsymbol{V}_{h, 0}^{n}-\boldsymbol{u}^{n}\right) \mathrm{d} x\right), \\
& \mathcal{S}_{2}=\Delta t \sum_{n=1}^{m} \sum_{K \in \mathcal{T}}|K| r_{K}^{n-1} \frac{\boldsymbol{V}_{h, 0, K}^{n}-\boldsymbol{V}_{h, 0, K}^{n-1}}{\Delta t} \cdot\left(\boldsymbol{V}_{h, 0, K}^{n}-\boldsymbol{u}_{K}^{n}\right), \\
& \mathcal{S}_{3}=\Delta t \sum_{n=1}^{m} \sum_{K \in \mathcal{T}} \sum_{\sigma \in \mathcal{E}(K)}|\sigma| r_{\sigma}^{n, \text { up }}\left(\hat{\boldsymbol{V}}_{h, 0, \sigma}^{n, \text { up }}-\hat{\boldsymbol{u}}_{\sigma}^{n, \text { up }}\right) \cdot\left(\boldsymbol{V}_{h, 0, \sigma}^{n}-\boldsymbol{V}_{h, 0, K}^{n}\right) \hat{\boldsymbol{V}}_{h, 0, \sigma}^{n, \text { up }} \cdot \boldsymbol{n}_{\sigma, K} \\
& \mathcal{S}_{4}=-\Delta t \sum_{n=1}^{m} \int_{\Omega_{h}} p\left(\hat{r}^{n}\right) \operatorname{div} \boldsymbol{V}^{n} \mathrm{~d} x, \\
& \mathcal{S}_{5}=0, \\
& \mathcal{S}_{6}=-\Delta t \sum_{n=1}^{m} \int_{\Omega_{h}} p^{\prime}\left(\hat{r}^{n}\right) \boldsymbol{u}^{n} \cdot \nabla r^{n} \mathrm{~d} x,
\end{aligned}
$$

and

$$
\left|\mathcal{R}_{h, \Delta t}^{m}\right| \leq c\left(h^{5 / 6}+\Delta t\right),
$$

for any couple $(r, \mathbf{V})$ belonging to (2.25) and satisfying the continuity equation (1.1) on $(0, T) \times \mathbb{R}^{3}$ and momentum equation (1.2) with boundary conditions (1.5) on $(0, T) \times \Omega$ in the classical sense.

Before starting the proof we recall an auxiliary algebraic inequality whose straightforward proof is left to the reader, and introduce some notations.

Lemma 7.2. Let $p$ satisfies assumptions (1.4). Let $0<a<b<\infty$. Then there exists $c=c(a, b)>0$ such that for all $\varrho \in[0, \infty)$ and $r \in[a, b]$ there holds

$$
E(\varrho \mid r) \geq c(a, b)\left(1_{R_{+} \backslash[a / 2,2 b]}(\varrho)+\varrho^{\gamma} 1_{R_{+} \backslash[a / 2,2 b]}(\varrho)+(\varrho-r)^{2} 1_{[a / 2,2 b]}(\varrho)\right),
$$

where $E(\varrho \mid r)$ is defined in 4.8$)$. 
If we take in Lemma $7.2 \varrho=\varrho^{n}(x), r=\hat{r}^{n}(x), a=\underline{r}, b=\bar{r}$ (where $\mathrm{r}$ is a function belonging to class (2.25) and $\underline{r}, \bar{r}$ are its lower and upper bounds, respectively), we obtain

$E\left(\varrho^{n}(x) \mid \hat{r}^{n}(x)\right) \geq c(\underline{r}, \bar{r})\left(1_{\left.R_{+} \backslash \underline{r} / 2,2 \bar{r}\right]}\left(\varrho^{n}(x)\right)+\left(\varrho^{n}\right)^{\gamma}(x) 1_{\left.R_{+} \backslash \underline{r} / 2,2 \bar{r}\right]}\left(\varrho^{n}(x)\right)+\left(\varrho^{n}(x)-\hat{r}^{n}(x)\right)^{2} 1_{[\underline{r} / 2,2 \bar{r}]}\left(\varrho^{n}(x)\right)\right)$.

Now, for fixed numbers $\underline{r}$ and $\bar{r}$ and fixed functions $\varrho^{n}, n=0, \ldots, N$, we introduce the residual and essential subsets of $\Omega$ (relative to $\varrho^{n}$ ) as follows:

$$
N_{\mathrm{ess}}^{n}=\left\{x \in \Omega \mid \frac{1}{2} \underline{r} \leq \varrho^{n}(x) \leq 2 \bar{r}\right\}, N_{\mathrm{res}}^{n}=\Omega \backslash N_{\mathrm{ess}}^{n},
$$

and we set

$$
[g]_{\mathrm{ess}}(x)=g(x) 1_{N_{\mathrm{ess}}^{n}}(x),[g]_{\mathrm{res}}(x)=g(x) 1_{N_{\mathrm{res}}^{n}}(x), \quad x \in \Omega, g \in L^{1}(\Omega) .
$$

Integrating inequality (7.2) we deduce

$$
c(\underline{r}, \bar{r}) \sum_{K \in \mathcal{T}} \int_{K}\left([1]_{\mathrm{res}}+\left[\left(\varrho^{n}\right)^{\gamma}\right]_{\mathrm{res}}+\left[\varrho^{n}-\hat{r}^{n}\right]_{\mathrm{ess}}^{2}\right) \mathrm{d} x \leq \mathcal{E}\left(\varrho^{n}, \boldsymbol{u}^{n} \mid \hat{r}^{n}, \boldsymbol{V}^{n}\right)
$$

for any pair $(r, \boldsymbol{V})$ belonging to the class (2.25) and any $\varrho^{n} \in Q_{h}\left(\Omega_{h}\right), \varrho^{n} \geq 0$.

We are now ready to proceed to the proof of Lemma 7.1.

Proof: Since $(r, \boldsymbol{V})$ satisfies (1.1) on $(0, T) \times \Omega$ and belongs to the class (2.25), Equation (1.2) can be rewritten in the form

$$
r \partial_{t} \boldsymbol{V}+r \boldsymbol{V} \cdot \nabla \boldsymbol{V}+\nabla p(r)-\mu \Delta \boldsymbol{V}-\mu / 3 \nabla \operatorname{div} \boldsymbol{V}=0 \text { in }(0, T) \times \Omega .
$$

From this fact, we deduce the identity

$$
\sum_{i=1}^{5} \mathcal{T}_{i}=\mathcal{R}_{0}
$$

where

$$
\begin{aligned}
& \mathcal{R}_{0}=\Delta t \sum_{n=1}^{m} \int_{\Omega_{h} \backslash \Omega}\left(r^{n}\left[\partial_{t} \boldsymbol{V}\right]^{n}+r \boldsymbol{V}^{n} \cdot \nabla \boldsymbol{V}^{n}+\nabla p\left(r^{n}\right)-\mu \Delta \boldsymbol{V}^{n}-\frac{\mu}{3} \nabla \operatorname{div} \boldsymbol{V}^{n}\right) \cdot\left(\boldsymbol{V}_{h, 0}^{n}-\mathbf{u}^{n}\right) \mathrm{d} x, \\
& \mathcal{T}_{1}=-\Delta t \sum_{n=1}^{m} \int_{\Omega_{h}}\left(\mu \Delta \boldsymbol{V}^{n}+\frac{\mu}{3} \nabla \operatorname{div} \boldsymbol{V}^{n}\right) \cdot\left(\boldsymbol{V}_{h, 0}^{n}-\boldsymbol{u}^{n}\right) \mathrm{d} x, \quad \mathcal{T}_{2}=\Delta t \sum_{n=1}^{m} \int_{\Omega_{h}} r^{n}\left[\partial_{t} \boldsymbol{V}\right]^{n} \cdot\left(\boldsymbol{V}_{h, 0}^{n}-\boldsymbol{u}^{n}\right) \mathrm{d} x, \\
& \mathcal{T}_{3}=\Delta t \sum_{n=1}^{m} \int_{\Omega_{h}} r^{n} \boldsymbol{V}^{n} \cdot \nabla \boldsymbol{V}^{n} \cdot\left(\boldsymbol{V}_{h, 0}^{n}-\boldsymbol{u}^{n}\right) \mathrm{d} x, \quad \mathcal{T}_{4}=\Delta t \sum_{n=1}^{m} \int_{\Omega_{h}} \nabla p\left(r^{n}\right) \cdot \boldsymbol{V}_{h, 0}^{n} \mathrm{~d} x, \\
& \mathcal{T}_{5}=0, \quad \mathcal{T}_{6}=-\Delta t \sum_{n=1}^{m} \int_{\Omega_{h}} \nabla p\left(r^{n}\right) \cdot \boldsymbol{u}^{n} \mathrm{~d} x .
\end{aligned}
$$

In the steps below, we deal with each of the terms $\mathcal{R}_{0}$ and $\mathcal{T}_{i}$.

Step 0: Term $\mathcal{R}_{0}$. By the Hölder inequality

$$
\begin{aligned}
& \qquad\left|\mathcal{R}_{0}\right| \leq\left|\Omega_{h} \backslash \Omega\right|^{5 / 6} c\left(\bar{r},\left|p^{\prime}\right|_{C[\underline{r}, \bar{r}]},\left\|\left(\partial_{t} r, \nabla r, \mathbf{V}, \nabla \mathbf{V}, \nabla^{2} \mathbf{V}\right)\right\|_{L^{\infty}\left(Q_{T} ; \mathbb{R}^{43}\right)} \Delta t \sum_{n=1}^{m}\left(\left\|\boldsymbol{u}^{n}\right\|_{L^{6}\left(\Omega_{h}\right)}+\left\|\boldsymbol{V}_{h, 0}^{n}\right\|_{L^{6}\left(\Omega_{h}\right)}\right)\right. \\
& \qquad \leq h^{5 / 3} c\left(M_{0}, E_{0}, \bar{r},\left|p^{\prime}\right|_{C[\underline{r}, \bar{r}]},\left\|\left(\partial_{t} r, \nabla r, \mathbf{V}, \nabla \mathbf{V}, \nabla^{2} \mathbf{V}\right)\right\|_{L^{\infty}\left(Q_{T} ; \mathbb{R}^{43}\right)}\right.
\end{aligned}
$$


Step 1: Term $\mathcal{T}_{1}$. Integrating by parts, we get:

$\mathcal{T}_{1}=\mathcal{T}_{1,1}+\mathcal{R}_{1,1}$,

with $\mathcal{T}_{1,1}=\Delta t \sum_{n=1}^{m} \sum_{K \in \mathcal{T}} \int_{K}\left(\mu \nabla \boldsymbol{V}_{h, 0}^{n}: \nabla\left(\boldsymbol{V}_{h, 0}^{n}-\boldsymbol{u}^{n}\right)+\frac{\mu}{3} \operatorname{div} \boldsymbol{V}_{h, 0}^{n} \operatorname{div}\left(\boldsymbol{V}_{h, 0}^{n}-\boldsymbol{u}^{n}\right)\right) \mathrm{d} x$,

and $\mathcal{R}_{1,1}=I_{1}+I_{2}$, with

$$
\begin{aligned}
& I_{1}=\Delta t \sum_{n=1}^{m} \sum_{K \in \mathcal{T}} \int_{K}\left(\mu \nabla\left(\boldsymbol{V}^{n}-\boldsymbol{V}_{h, 0}^{n}\right): \nabla\left(\boldsymbol{V}_{h, 0}^{n}-\boldsymbol{u}^{n}\right)+\frac{\mu}{3} \operatorname{div}\left(\boldsymbol{V}^{n}-\boldsymbol{V}_{h, 0}^{n}\right) \operatorname{div}\left(\boldsymbol{V}_{h, 0}^{n}-\boldsymbol{u}^{n}\right)\right) \mathrm{d} x, \\
& I_{2}=-\Delta t \sum_{n=1}^{m} \sum_{K \in \mathcal{T}} \sum_{\sigma \in \mathcal{E}(K)} \int_{\sigma}\left(\mu \boldsymbol{n}_{\sigma, K} \cdot \nabla \boldsymbol{V}^{n} \cdot\left(\boldsymbol{V}_{h, 0}^{n}-\boldsymbol{u}^{n}\right)+\frac{\mu}{3} \operatorname{div} \boldsymbol{V}^{n}\left(\boldsymbol{V}_{h, 0}^{n}-\boldsymbol{u}^{n}\right) \cdot \boldsymbol{n}_{\sigma, K}\right) \mathrm{d} S \\
& =-\Delta t \sum_{n=1}^{m} \sum_{\sigma \in \mathcal{E}} \int_{\sigma}\left(\mu \boldsymbol{n}_{\sigma} \cdot \nabla \boldsymbol{V}^{n} \cdot\left[\boldsymbol{V}_{h, 0}^{n}-\boldsymbol{u}^{n}\right]_{\sigma, \mathbf{n}_{\sigma}}+\frac{\mu}{3} \operatorname{div} \boldsymbol{V}^{n}\left[\boldsymbol{V}_{h, 0}^{n}-\boldsymbol{u}^{n}\right]_{\sigma, \mathbf{n}_{\sigma}} \cdot \boldsymbol{n}_{\sigma}\right) \mathrm{d} S,
\end{aligned}
$$

where in the last line $\mathbf{n}_{\sigma}$ is the unit normal to the face $\sigma$ and $[\cdot]_{\sigma, \mathbf{n}_{\sigma}}$ is the jump over sigma (with respect to $\mathbf{n}_{\sigma}$ ) defined in Lemma 2.7.

To estimate $I_{1}$, we use the Cauchy-Schwartz inequality, decompose $\mathbf{V}^{n}-\mathbf{V}_{h, 0}^{n}=\mathbf{V}^{n}-\mathbf{V}_{h}^{n}+\mathbf{V}_{h}^{n}-$ $\mathbf{V}_{h, 0}^{n}$ and employ estimates (2.40) $)_{s=2}$, (2.472.48) to evaluate the norms involving $\nabla\left(\mathbf{V}^{n}-\mathbf{V}_{h, 0}^{n}\right)$, and decompose $\mathbf{V}_{h, 0}^{n}=\mathbf{V}_{h, 0}^{n}-\mathbf{V}_{h}^{n}+\mathbf{V}_{h}^{n}$ use (2.472.48), (2.39) $)_{s=1}$, (4.4), the Minkowski inequalityt to estimate the norms involving $\nabla\left(\mathbf{V}_{h, 0}^{n}-\mathbf{u}^{n}\right)$. We get

$$
\left|I_{1}\right| \leq h c\left(M_{0}, E_{0},\left\|\nabla \mathbf{V}, \nabla^{2} \boldsymbol{V}\right\|_{L^{\infty}\left(0, T ; L^{\infty}\left(\Omega ; \mathbb{R}^{36}\right)\right)}\right) .
$$

Since the integral over any face $\sigma \in \mathcal{E}_{\text {int }}$ of the jump of a function from $V_{h, 0}\left(\Omega_{h}\right)$ is zero, we may write

$$
\begin{aligned}
I_{2}=\Delta & t \sum_{n=1}^{m} \sum_{\sigma \in \mathcal{E}_{\text {int }}} \int_{\sigma}\left(\mu \boldsymbol{n}_{\sigma} \cdot\left(\nabla \boldsymbol{V}^{n}-\left(\nabla \boldsymbol{V}^{n}\right)_{\sigma}\right) \cdot\left[\boldsymbol{u}^{n}-\boldsymbol{V}_{h, 0}^{n}\right]_{\sigma, \mathbf{n}_{\sigma}}\right. \\
& \left.+\frac{\mu}{3}\left(\operatorname{div} \boldsymbol{V}^{n}-\left(\operatorname{div} \boldsymbol{V}^{n}\right)_{\sigma}\right)\left[\boldsymbol{u}^{n}-\boldsymbol{V}_{h, 0}^{n}\right]_{\sigma, \mathbf{n}_{\sigma}} \cdot \boldsymbol{n}_{\sigma}\right) \mathrm{d} S ;
\end{aligned}
$$

whence by using the first order Taylor formula applied to functions $x \mapsto \nabla \mathbf{V}^{n}(x)$ to evaluate the differences $\nabla \boldsymbol{V}^{n}-\left(\nabla \boldsymbol{V}^{n}\right)_{\sigma}, \operatorname{div} \boldsymbol{V}^{n}-\left[\operatorname{div} \boldsymbol{V}^{n}\right]_{\sigma}$, and Hölder's inequality,

$$
\begin{aligned}
& \left|I_{2}\right| \leq \Delta t h c\left\|\nabla^{2} \boldsymbol{V}\right\|_{L^{\infty}\left(Q_{T} ; \mathbb{R}^{27}\right)} \sum_{n=1}^{m} \sum_{\sigma \in \mathcal{E}_{\text {int }}} \sqrt{|\sigma|} \sqrt{h}\left(\frac{1}{\sqrt{h}}\left\|\left[\boldsymbol{u}^{n}-\boldsymbol{V}_{h, 0}^{n}\right]_{\sigma, \mathbf{n}_{\sigma}}\right\|_{L^{2}\left(\sigma ; \mathbb{R}^{3}\right)}\right) \\
& \leq \Delta t h c\left\|\nabla^{2} \boldsymbol{V}\right\|_{L^{\infty}\left(Q_{T} ; \mathbb{R}^{27}\right)} \sum_{n=1}^{m} \sum_{\sigma \in \mathcal{E}_{\text {int }}}\left(|\sigma| h+\frac{1}{h}\left\|\left[\boldsymbol{u}^{n}-\boldsymbol{V}_{h, 0}^{n}\right]_{\sigma, \mathbf{n}_{\sigma}}\right\|_{L^{2}\left(\sigma ; \mathbb{R}^{3}\right)}^{2}\right) .
\end{aligned}
$$

Therefore,

$$
\left|\mathcal{R}_{1,1}\right| \leq h c\left(M_{0}, E_{0},\left\|\mathbf{V}, \nabla \boldsymbol{V}, \nabla^{2} \boldsymbol{V}\right\|_{L^{\infty}\left(Q_{T}, \mathbb{R}^{39}\right)}\right),
$$

where we have employed Lemma 2.7, (4.4) and (2.47 2.48), (2.39).

Step 2: Term $\mathcal{T}_{2}$. Let us now decompose the term $\mathcal{T}_{2}$ as $\mathcal{T}_{2}=\mathcal{T}_{2,1}+\mathcal{R}_{2,1}$,

with $\mathcal{T}_{2,1}=\Delta t \sum_{n=1}^{m} \sum_{K \in \mathcal{T}} \int_{K} r^{n-1} \frac{\boldsymbol{V}^{n}-\boldsymbol{V}^{n-1}}{\Delta t} \cdot\left(\boldsymbol{V}_{h, 0}^{n}-\boldsymbol{u}^{n}\right) \mathrm{d} x, \quad \mathcal{R}_{2,1}=\Delta t \sum_{n=1}^{m} \sum_{K \in \mathcal{T}} \mathcal{R}_{2,1}^{n, K}$,

and $\mathcal{R}_{2,1}^{n, K}=\int_{K}\left(r^{n}-r^{n-1}\right)\left[\partial_{t} \mathbf{V}\right]^{n} \cdot\left(\boldsymbol{V}_{h, 0}^{n}-\boldsymbol{u}^{n}\right) \mathrm{d} x+\int_{K} r^{n-1}\left(\left[\partial_{t} \boldsymbol{V}\right]^{n}-\frac{\boldsymbol{V}^{n}-\boldsymbol{V}^{n-1}}{\Delta t}\right) \cdot\left(\boldsymbol{V}_{h, 0}^{n}-\boldsymbol{u}^{n}\right) \mathrm{d} x$. 
The remainder $\mathcal{R}_{2,1}^{n, K}$ can be rewritten as follows

$\mathcal{R}_{2,1}^{n, K}=\int_{K}\left[\int_{t_{n-1}}^{t_{n}} \partial_{t} r(t, \cdot) \mathrm{d} t\right]\left[\partial_{t} \mathbf{V}\right]^{n} \cdot\left(\boldsymbol{V}_{h, 0}^{n}-\boldsymbol{u}^{n}\right) \mathrm{d} x+\frac{1}{\Delta t} \int_{K} r^{n-1}\left[\int_{t_{n-1}}^{t_{n}} \int_{s}^{t_{n}} \partial_{t}^{2} \boldsymbol{V}(z, \cdot) \mathrm{d} z \mathrm{~d} s\right] \cdot\left(\boldsymbol{V}_{h, 0}^{n}-\boldsymbol{u}^{n}\right) \mathrm{d} x ;$

whence, by the Hölder inequality,

$$
\begin{gathered}
\left|\mathcal{R}_{2,1}^{n, K}\right| \leq \Delta t\left[( \| r \| _ { L ^ { \infty } ( Q _ { T } ) } + \| \partial _ { t } r \| _ { L ^ { \infty } ( Q _ { T } ) } ) \left(\left\|\partial_{t} \boldsymbol{V}\right\|_{L^{\infty}\left(Q_{T} ; \mathbb{R}^{3}\right)}|K|^{5 / 6}\left(\left\|\boldsymbol{u}^{n}\right\|_{L^{6}(K)}+\left\|\boldsymbol{V}_{h, 0}^{n}\right\|_{L^{6}(K)}\right)\right.\right. \\
\left.+\left\|\partial_{t}^{2} \boldsymbol{V}^{n}\right\|_{\left.L^{6 / 5}\left(\Omega ; \mathbb{R}^{3}\right)\right)}\left(\left\|\boldsymbol{u}^{n}\right\|_{L^{6}(K)}+\left\|\boldsymbol{V}_{h, 0}^{n}\right\|_{L^{6}(K)}\right)\right] .
\end{gathered}
$$

Consequently, by the same token as in (6.19) or (6.23),

$$
\left|\mathcal{R}_{2,1}\right| \leq \Delta t c\left(M_{0}, E_{0}, \bar{r},\left\|\left(\partial_{t} r, \boldsymbol{V}, \partial_{t} \boldsymbol{V}, \nabla \boldsymbol{V}\right)\right\|_{L^{\infty}\left(Q_{T} ; \mathbb{R}^{16}\right)},\left\|\partial_{t}^{2} \boldsymbol{V}\right\|_{L^{2}\left(0, T ; L^{6 / 5}\left(\Omega ; \mathbb{R}^{3}\right)\right)}\right),
$$

where we have used the discrete Hölder and Young inequalities, the estimates (2.38), (2.47 2.48) and the energy bound (4.4) from Corollary 4.1.

Step 2a: $\operatorname{Term} \mathcal{T}_{2,1}$. We decompose the term $\mathcal{T}_{2,1}$ as

$$
\begin{aligned}
& \mathcal{T}_{2,1}=\mathcal{T}_{2,2}+\mathcal{R}_{2,2}, \\
& \text { with } \mathcal{T}_{2,2}=\Delta t \sum_{n=1}^{m} \sum_{K \in \mathcal{T}} \int_{K} r_{K}^{n-1} \frac{\boldsymbol{V}^{n}-\boldsymbol{V}^{n-1}}{\Delta t} \cdot\left(\boldsymbol{V}_{h, 0}^{n}-\boldsymbol{u}^{n}\right) \mathrm{d} x, \mathcal{R}_{2,2}=\Delta t \sum_{n=1}^{m} \sum_{K \in \mathcal{T}} \mathcal{R}_{2,2}^{n, K}, \\
& \text { and } \mathcal{R}_{2,2}^{n, K}=\int_{K}\left(r^{n-1}-r_{K}^{n-1}\right) \frac{\boldsymbol{V}^{n}-\boldsymbol{V}^{n-1}}{\Delta t} \cdot\left(\boldsymbol{V}_{h, 0}^{n}-\boldsymbol{u}^{n}\right) \mathrm{d} x ;
\end{aligned}
$$

therefore,

$$
\left|\mathcal{R}_{2,2}^{n}\right|=\left|\sum_{K \in \mathcal{T}} \mathcal{R}_{2,2}^{n, K}\right| \leq h c\|\nabla r\|_{L^{\infty}\left(Q_{T} ; \mathbb{R}^{3}\right)}\left\|\partial_{t} \boldsymbol{V}\right\|_{L^{\infty}\left(Q_{T} ; \mathbb{R}^{3}\right)}\left\|\boldsymbol{u}^{n}-\boldsymbol{V}_{h, 0}^{n}\right\|_{L^{6}\left(\Omega ; \mathbb{R}^{3}\right)} .
$$

Consequently, by virtue of formula (4.5) for $\mathbf{u}^{n}$ and estimates (2.38), (2.47 2.48),

$$
\left|\mathcal{R}_{2,2}\right| \leq h c\left(M_{0}, E_{0},\left\|\left(\nabla r, \boldsymbol{V}, \partial_{t} \boldsymbol{V}, \nabla \boldsymbol{V}\right)\right\|_{L^{\infty}\left(Q_{T} ; \mathbb{R}^{18}\right)}\right) .
$$

Step 2b: $\operatorname{Term} \mathcal{T}_{2,2}$. We decompose the term $\mathcal{T}_{2,2}$ as

$$
\begin{aligned}
& \mathcal{T}_{2,2}=\mathcal{T}_{2,3}+\mathcal{R}_{2,3}, \\
& \text { with } \mathcal{T}_{2,3}=\Delta t \sum_{n=1}^{m} \sum_{K \in \mathcal{T}} \int_{K} r_{K}^{n-1} \frac{\boldsymbol{V}_{h, 0, K}^{n}-\boldsymbol{V}_{h, 0, K}^{n-1}}{\Delta t} \cdot\left(\boldsymbol{V}_{h, 0}^{n}-\boldsymbol{u}^{n}\right) \mathrm{d} x, \mathcal{R}_{2,3}=\Delta t \sum_{n=1}^{m} \sum_{K \in \mathcal{T}} \mathcal{R}_{2,3}^{n, K}, \\
& \text { and } \mathcal{R}_{2,3}^{n, K}=\int_{K} r_{K}^{n-1}\left(\frac{\boldsymbol{V}^{n}-\boldsymbol{V}^{n-1}}{\Delta t}-\left[\frac{\boldsymbol{V}^{n}-\boldsymbol{V}^{n-1}}{\Delta t}\right]_{h}\right) \cdot\left(\boldsymbol{V}_{h, 0}^{n}-\boldsymbol{u}^{n}\right) \mathrm{d} x \\
& +\int_{K} r_{K}^{n-1}\left(\left[\frac{\boldsymbol{V}^{n}-\boldsymbol{V}^{n-1}}{\Delta t}\right]_{h}-\left[\frac{\boldsymbol{V}^{n}-\boldsymbol{V}^{n-1}}{\Delta t}\right]_{h, K}\right) \cdot\left(\boldsymbol{V}_{h, 0}^{n}-\boldsymbol{u}^{n}\right) \mathrm{d} x \\
& +\int_{K} r_{K}^{n-1}\left(\left[\frac{\boldsymbol{V}^{n}-\boldsymbol{V}^{n-1}}{\Delta t}\right]_{h, K}-\left[\frac{\boldsymbol{V}^{n}-\boldsymbol{V}^{n-1}}{\Delta t}\right]_{h, 0, K}\right) \cdot\left(\boldsymbol{V}_{h, 0}^{n}-\boldsymbol{u}_{n}\right) \mathrm{d} x=I_{1}^{K}+I_{2}^{K}+I_{3}^{K} .
\end{aligned}
$$

We calculate carefully

$$
\left|I_{3}^{K}\right|=\frac{1}{\Delta t} r_{K}^{n-1} \int_{K}\left\{\int_{t_{n-1}}^{t_{n}}\left[\left[\partial_{t} \boldsymbol{V}(z)\right]_{h}-\left[\partial_{t} \boldsymbol{V}(z)\right]_{h, 0}\right]_{K} \cdot\left(\boldsymbol{V}_{h, 0}^{n}-\boldsymbol{u}^{n}\right) \mathrm{d} z\right\} \mathrm{d} x
$$




$$
\leq \frac{1}{\Delta t} r_{K}^{n-1} \int_{t_{n-1}}^{t_{n}}\left\|\left[\left[\partial_{t} \boldsymbol{V}(z)\right]_{h}-\left[\partial_{t} \boldsymbol{V}(z)\right]_{h, 0}\right]_{K}\right\|_{L^{6 / 5}\left(K ; \mathbb{R}^{3}\right)}\left\|\boldsymbol{V}_{h, 0}^{n}-\boldsymbol{u}^{n}\right\|_{L^{6}\left(K ; \mathbb{R}^{3}\right)} \mathrm{d} z .
$$

Summing over polyhedra $K \in \mathcal{T}$ we get simply by using the discrete Sobolev inequality

$$
\begin{gathered}
\sum_{K \in \mathcal{T}}\left|I_{3}^{K}\right| \leq \frac{1}{\Delta t} r_{K}^{n-1} \int_{t_{n-1}}^{t_{n}}\left\{\left(\sum_{K \in \mathcal{T}}\left\|\boldsymbol{V}_{h, 0}^{n}-\boldsymbol{u}^{n}\right\|_{L^{6}\left(K ; \mathbb{R}^{3}\right)}^{6}\right)^{1 / 6}\left(\sum_{K \in \mathcal{T}}\left\|\left[\partial_{t} \boldsymbol{V}(z)\right]_{h}-\left[\partial_{t} \boldsymbol{V}(z)\right]_{h, 0}\right\|_{L^{6 / 5}\left(K ; \mathbb{R}^{3}\right)}^{6 / 5}\right)^{5 / 6}\right\} \mathrm{d} z \\
\leq \frac{1}{\Delta t} r_{K}^{n-1} \int_{t_{n-1}}^{t_{n}}\left\|\boldsymbol{V}_{h, 0}^{n}-\boldsymbol{u}^{n}\right\|_{L^{6}\left(\Omega_{h} ; \mathbb{R}^{3}\right)}\left\|\left[\partial_{t} \boldsymbol{V}(z)\right]_{h}-\left[\partial_{t} \boldsymbol{V}(z)\right]_{h, 0}\right\|_{L^{6 / 5}\left(\Omega_{h} ; \mathbb{R}^{3}\right)} \mathrm{d} z \\
\leq \frac{h^{5 / 6}}{\Delta t} \int_{t_{n-1}}^{t_{n}}\left\|\boldsymbol{V}_{h, 0}^{n}-\boldsymbol{u}^{n}\right\|_{L^{6}\left(\Omega_{h} ; \mathbb{R}^{3}\right)}\left\|\partial_{t} \boldsymbol{V}(z)\right\|_{L^{\infty}\left(\Omega_{h} ; \mathbb{R}^{3}\right)} \mathrm{d} z
\end{gathered}
$$

where we have used estimate (2.50) to obtain the last line.

As far as the term $I_{2}^{K}$ is concerned, we write

$$
\begin{gathered}
\left|I_{2}^{K}\right|=\frac{1}{\Delta t} r_{K}^{n-1}\left|\int_{K}\left(\left[\int_{t_{n-1}}^{t_{n}} \partial_{t} \boldsymbol{V}(z) \mathrm{d} z\right]_{h}-\left[\int_{t_{n-1}}^{t_{n}} \partial_{t} \boldsymbol{V}(z) \mathrm{d} z\right]_{h, K}\right) \cdot\left(\boldsymbol{u}^{n}-\boldsymbol{V}_{h, 0}^{n}\right) \mathrm{d} x\right| \\
\leq \frac{h}{\Delta t} r_{K}^{n-1} \int_{t_{n-1}}^{t_{n}}\left\|\nabla_{x}\left[\partial_{t} \boldsymbol{V}(z)\right]_{h}\right\|_{L^{6 / 5}\left(K ; \mathbb{R}^{3}\right)}\left\|\boldsymbol{u}^{n}-\boldsymbol{V}_{h, 0}^{n}\right\|_{L^{6}\left(K ; \mathbb{R}^{3}\right)},
\end{gathered}
$$

where we have used the Fubini theorem, Hölder's inequality and (2.51), (2.40) $s=1$. Further, employing the Sobolev inequality on the Crouzeix-Raviart space $V_{h}\left(\Omega_{h}\right)(2.42)$, the Hölder inequality and estimate (2.40) $s=1$, we get

$$
\sum_{K \in \mathcal{T}}\left|I_{2}^{K}\right| \leq \frac{h}{\Delta t} r_{K}^{n-1}\left\|\boldsymbol{u}^{n}-\boldsymbol{V}_{h, 0}^{n}\right\|_{L^{6}\left(\Omega_{h} ; \mathbb{R}^{3}\right)} \int_{t_{n-1}}^{t_{n}}\left\|\nabla_{x} \partial_{t} \boldsymbol{V}(z)\right\|_{L^{6 / 5}\left(\Omega_{h} ; \mathbb{R}^{3}\right)} \mathrm{d} z .
$$

We reserve the similar treatment to the term $I_{1}^{K}$. Resuming these calculations and summing over $n$ from 1 to $m$ we get by using Corollary 4.1 and estimates (2.47 2.48), (2.38),

$$
\left|\mathcal{R}_{2,3}\right| \leq h^{5 / 6} c\left(M_{0}, E_{0},\left\|\left(r, \boldsymbol{V}, \nabla \boldsymbol{V}, \partial_{t} \boldsymbol{V}\right)\right\|_{L^{\infty}\left(Q_{T} ; \mathbb{R}^{16}\right)},\left\|\partial_{t} \nabla \boldsymbol{V}\right\|_{L^{2}\left(0, T ; L^{6 / 5}\left(\Omega ; \mathbb{R}^{9}\right)\right)}\right) .
$$

Step 2c: $\operatorname{Term} \mathcal{T}_{2,3}$. We rewrite this term in the form

$$
\begin{aligned}
& \mathcal{T}_{2,3}=\mathcal{T}_{2,4}+\mathcal{R}_{2,4}, \mathcal{R}_{2,4}=\Delta t \sum_{n=1}^{m} \sum_{K \in \mathcal{T}} \mathcal{R}_{2,4}^{n, K} \\
& \text { with } \mathcal{T}_{2,4}=\Delta t \sum_{n=1}^{m} \sum_{K \in \mathcal{T}} \int_{K} r_{K}^{n-1} \frac{\boldsymbol{V}_{h, 0, K}^{n}-\boldsymbol{V}_{h, 0, K}^{n-1}}{\Delta t} \cdot\left(\boldsymbol{u}_{K}^{n}-\boldsymbol{V}_{h, 0, K}^{n}\right) \mathrm{d} x \\
& \text { and } \mathcal{R}_{2,4}^{n, K}=\int_{K} r_{K}^{n-1} \frac{\boldsymbol{V}_{h, 0, K}^{n}-\boldsymbol{V}_{h, 0, K}^{n-1}}{\Delta t} \cdot\left(\left(\boldsymbol{u}^{n}-\boldsymbol{u}_{K}^{n}\right)-\left(\boldsymbol{V}_{h, 0}^{n}-\boldsymbol{V}_{h, 0, K}^{n}\right)\right) \mathrm{d} x
\end{aligned}
$$

First, we estimate the $L^{\infty}$ norm of $\frac{\boldsymbol{V}_{h, 0, K}^{n}-\boldsymbol{V}_{h, 0, K}^{n-1}}{\Delta t}$ as in (6.5). Next, we decompose

$$
\boldsymbol{V}_{h, 0}^{n}-\boldsymbol{V}_{h, 0, K}^{n}=\boldsymbol{V}_{h, 0}^{n}-\boldsymbol{V}_{h}^{n}+\boldsymbol{V}_{h}^{n}-\boldsymbol{V}_{h, K}^{n}+\left[\boldsymbol{V}_{h}^{n}-\boldsymbol{V}_{h, 0}^{n}\right]_{K},
$$

and use (2.51) $)_{p=2}$ to estimate $\boldsymbol{u}^{n}-\boldsymbol{u}_{K}^{n},(2.51)_{p=\infty},(2.40)_{s=1}$ to estimate $\boldsymbol{V}_{h}^{n}-\boldsymbol{V}_{h, K}^{n}$ and (2.472.48) to evaluate $\left\|\left[\boldsymbol{V}_{h}^{n}-\boldsymbol{V}_{h, 0}^{n}\right]_{K}\right\|_{L^{\infty}\left(K ; \mathbb{R}^{3}\right)} \leq\left\|\boldsymbol{V}_{h}^{n}-\boldsymbol{V}_{h, 0}^{n}\right\|_{L^{\infty}\left(K ; \mathbb{R}^{3}\right)}$. Thanks to the Hölder inequality and (4.4) we finally deduce

$$
\left|\mathcal{R}_{2,4}\right| \leq h c\left(M_{0}, E_{0}, \bar{r},\left\|\left(\boldsymbol{V}, \partial_{t} \boldsymbol{V}, \nabla \boldsymbol{V}\right)\right\|_{L^{\infty}\left(Q_{T} ; \mathbb{R}^{15}\right)}\right) .
$$


Step 3: $\quad \operatorname{Term} \mathcal{T}_{3}$. Let us first decompose $\mathcal{T}_{3}$ as

$\mathcal{T}_{3}=\mathcal{T}_{3,1}+\mathcal{R}_{3,1}$,

with $\mathcal{T}_{3,1}=\Delta t \sum_{n=1}^{m} \sum_{K \in \mathcal{T}} \int_{K} r_{K}^{n} \boldsymbol{V}_{h, 0, K}^{n} \cdot \nabla \boldsymbol{V}^{n} \cdot\left(\boldsymbol{V}_{h, 0, K}^{n}-\boldsymbol{u}_{K}^{n}\right) \mathrm{d} x, \quad \mathcal{R}_{3,1}=\Delta t \sum_{n=1}^{m} \sum_{K \in \mathcal{T}} \mathcal{R}_{3,1}^{n, K}$,

and $\mathcal{R}_{3,1}^{n, K}=\int_{K}\left(r^{n}-r_{K}^{n}\right) \boldsymbol{V}^{n} \cdot \nabla \boldsymbol{V}^{n} \cdot\left(\boldsymbol{V}_{h, 0}^{n}-\boldsymbol{u}^{n}\right) \mathrm{d} x+\int_{K} r_{K}^{n}\left(\boldsymbol{V}^{n}-\boldsymbol{V}_{h, 0}^{n}\right) \cdot \nabla \boldsymbol{V}^{n} \cdot\left(\boldsymbol{V}_{h, 0}^{n}-\boldsymbol{u}^{n}\right) \mathrm{d} x$

$+\int_{K} r_{K}^{n}\left(\boldsymbol{V}_{h, 0}^{n}-\boldsymbol{V}_{h, 0, K}^{n}\right) \cdot \nabla \boldsymbol{V}^{n} \cdot\left(\boldsymbol{V}_{h, 0}^{n}-\boldsymbol{u}^{n}\right) \mathrm{d} x$

$+\int_{K} r_{K}^{n} \boldsymbol{V}_{h, 0, K}^{n} \cdot \nabla \boldsymbol{V}^{n} \cdot\left(\boldsymbol{V}_{h, 0}^{n}-\boldsymbol{V}_{h, 0, K}^{n}-\left(\boldsymbol{u}^{n}-\boldsymbol{u}_{K}^{n}\right)\right) \mathrm{d} x$.

We have

$$
\left\|r^{n}-r_{K}^{n}\right\|_{L^{\infty}(K)} \lesssim h\left\|\nabla r^{n}\right\|_{L^{\infty}(K)}
$$

by the Taylor formula,

$$
\left\|\boldsymbol{V}^{n}-\boldsymbol{V}_{h, 0}^{n}\right\|_{L^{\infty}\left(K ; \mathbb{R}^{3}\right)} \lesssim h\left\|\nabla \boldsymbol{V}^{n}\right\|_{L^{\infty}\left(K ; \mathbb{R}^{9}\right)},
$$

by virtue of $(2.39) s_{s=1}$ and $(2.47 \sqrt{2.48})$,

$$
\begin{aligned}
& \left\|\boldsymbol{V}_{h, 0}^{n}-\boldsymbol{V}_{h, 0, K}^{n}\right\|_{L^{\infty}\left(K ; \mathbb{R}^{3}\right)} \leq\left\|\boldsymbol{V}_{h, 0}^{n}-\boldsymbol{V}_{h, \|^{\infty}\left(K ; \mathbb{R}^{3}\right)}^{n}+\right\| \boldsymbol{V}_{h}^{n}-\boldsymbol{V}_{h, K}^{n} \|_{L^{\infty}\left(K ; \mathbb{R}^{3}\right)} \\
& +\left\|\left[\boldsymbol{V}_{h}^{n}-\boldsymbol{V}_{h, 0}^{n}\right]_{K}\right\|_{L^{\infty}\left(K ; \mathbb{R}^{3}\right)} \lesssim h\left\|\nabla \boldsymbol{V}^{n}\right\|_{L^{\infty}\left(K ; \mathbb{R}^{9}\right)}
\end{aligned}
$$

by virtue of (2.51), (2.39) $s=1(2.40) s=1$ and $(2.47 \sqrt{2.48})$,

$$
\left\|\boldsymbol{u}^{n}-\boldsymbol{u}_{K}^{n}\right\|_{L^{\infty}\left(K ; \mathbb{R}^{3}\right)} \lesssim h\left\|\nabla \boldsymbol{u}^{n}\right\|_{L^{\infty}\left(K ; \mathbb{R}^{9}\right)} \cdot
$$

Consequently by employing several times the Hölder inequality (for integrals over $K$ ) and the discrete Hölder inequality (for the sums over $K \in \mathcal{T}$ ), and using estimate (4.4), we arrive at

$$
\left|\mathcal{R}_{3,1}\right| \leq h c\left(M_{0}, E_{0}, \bar{r},\|(\nabla r, \boldsymbol{V}, \nabla \boldsymbol{V})\|_{L^{\infty}\left(Q_{T} ; \mathbb{R}^{15}\right)}\right) .
$$

Now we shall deal wit term $\mathcal{T}_{3,1}$. Integrating by parts, we get:

$$
\begin{aligned}
\int_{K} r_{K}^{n} \boldsymbol{V}_{h, 0, K}^{n} \cdot \nabla \boldsymbol{V}^{n} \cdot\left(\boldsymbol{V}_{h, 0, K}^{n}-\boldsymbol{u}_{K}^{n}\right) \mathrm{d} x & =\sum_{\sigma \in \mathcal{E}(K)}|\sigma| r_{K}^{n}\left[\boldsymbol{V}_{h, 0, K}^{n} \cdot \mathbf{n}_{\sigma, K}\right] \boldsymbol{V}_{\sigma}^{n} \cdot\left(\boldsymbol{V}_{h, 0, K}^{n}-\boldsymbol{u}_{K}^{n}\right) \\
& =\sum_{\sigma \in \mathcal{E}(K)}|\sigma| r_{K}^{n}\left[\boldsymbol{V}_{h, 0, K}^{n} \cdot \mathbf{n}_{\sigma, K}\right]\left(\boldsymbol{V}_{\sigma}^{n}-\boldsymbol{V}_{h, K}^{n}\right) \cdot\left(\boldsymbol{V}_{h, K}^{n}-\boldsymbol{u}_{K}^{n}\right),
\end{aligned}
$$

thanks to the the fact that $\sum_{\sigma \in \mathcal{E}(K)} \int_{\sigma} \boldsymbol{V}_{h, K}^{n} \cdot \mathbf{n}_{\sigma, K} \mathrm{~d} S=0$.

Next we write

$$
\begin{gathered}
\mathcal{T}_{3,1}=\mathcal{T}_{3,2}+\mathcal{R}_{3,2}, \quad \mathcal{R}_{3,2}=\Delta t \sum_{n=1}^{m} \mathcal{R}_{3,2}^{n}, \\
\mathcal{T}_{3,2}=\Delta t \sum_{n=1}^{m} \sum_{K \in \mathcal{T}} \sum_{\sigma \in \mathcal{E}(K)}|\sigma| \hat{r}_{\sigma}^{n, \text { up }}\left[\hat{\boldsymbol{V}}_{h, 0, \sigma}^{n, \text { up }} \cdot \mathbf{n}_{\sigma, K}\right]\left(\boldsymbol{V}_{\sigma}^{n}-\boldsymbol{V}_{h, K}^{n}\right) \cdot\left(\hat{\boldsymbol{V}}_{h, 0, \sigma}^{n, \text { up }}-\hat{\boldsymbol{u}}_{\sigma}^{n, \text { up }}\right),
\end{gathered}
$$




$$
\begin{aligned}
& \text { and } \mathcal{R}_{3,2}^{n}=\sum_{K \in \mathcal{T}} \sum_{\sigma \in \mathcal{E}(K)}|\sigma|\left(r_{K}^{n}-\hat{r}_{\sigma}^{n, \text { up }}\right)\left[\boldsymbol{V}_{h, 0, K}^{n} \cdot \mathbf{n}_{\sigma, K}\right]\left(\boldsymbol{V}_{\sigma}^{n}-\boldsymbol{V}_{h, K}^{n}\right) \cdot\left(\boldsymbol{V}_{h, 0, K}^{n}-\boldsymbol{u}_{K}^{n}\right) \\
& +\sum_{K \in \mathcal{T}} \sum_{\sigma \in \mathcal{E}(K)}|\sigma| \hat{r}_{\sigma}^{n, \text { up }}\left[\left(\boldsymbol{V}_{h, 0, K}^{n}-\hat{\boldsymbol{V}}_{h, 0, \sigma}^{n, \mathrm{up}}\right) \cdot \mathbf{n}_{\sigma, K}\right]\left(\boldsymbol{V}_{\sigma}^{n}-\boldsymbol{V}_{h, K}^{n}\right) \cdot\left(\boldsymbol{V}_{h, K}^{n}-\boldsymbol{u}_{K}^{n}\right) \\
& +\sum_{K \in \mathcal{T}} \sum_{\sigma \in \mathcal{E}(K)}|\sigma| \hat{r}_{\sigma}^{n, \text { up }}\left[\hat{\boldsymbol{V}}_{h, 0, \sigma}^{n, \text { up }} \cdot \mathbf{n}_{\sigma, K}\right]\left(\boldsymbol{V}_{\sigma}^{n}-\boldsymbol{V}_{h, K}^{n}\right) \cdot\left(\left(\boldsymbol{V}_{h, 0, K}^{n}-\hat{\boldsymbol{V}}_{h, 0, \sigma}^{n, \text { up }}\right)-\left(\boldsymbol{u}_{K}^{n}-\hat{\boldsymbol{u}}_{h, \sigma}^{n, \mathrm{up}}\right)\right) .
\end{aligned}
$$

We may write

$$
\mathbf{V}_{\sigma}^{n}-\mathbf{V}_{h, 0, K}^{n}=\mathbf{V}_{\sigma}^{n}-\mathbf{V}^{n}+\mathbf{V}^{n}-\mathbf{V}_{h}^{n}+\mathbf{V}_{h}^{n}-\mathbf{V}_{h, K}^{n}+\left[\mathbf{V}_{h}^{n}-\mathbf{V}_{h, 0}^{n}\right]_{K},
$$

and use several times the Taylor formula along with (2.39) $s_{s=1}$, (2.51), (2.40) $)_{s=1}$, (2.47 2.48) (in order to estimate $\left.r_{K}^{n}-\hat{r}_{\sigma}^{n, \text { up }}, \mathbf{V}_{\sigma}^{n}-\mathbf{V}_{h, 0, K}^{n}, \mathbf{V}_{h, K}^{n}-\hat{\mathbf{V}}_{h, \sigma}^{n, \text { up }}\right)$ to get the bound

$$
\begin{gathered}
\left|\mathcal{R}_{3,2}^{n}\right| \leq h c\|r\|_{W^{1, \infty}(\Omega)}\left(1+\|\boldsymbol{V}\|_{W^{1, \infty}\left(Q_{T} ; \mathbb{R}^{3}\right)}\right)^{3} \sum_{K \in \mathcal{T}} h|\sigma|\left|\boldsymbol{u}_{K}^{n}\right| \\
+c\|r\|_{W^{1, \infty}(\Omega)}\left(1+\|\boldsymbol{V}\|_{W^{1, \infty}\left(Q_{T} ; \mathbb{R}^{3}\right)}\right)^{2} \sum_{K \in \mathcal{T}} \sum_{\sigma \in \mathcal{E}(K)} h|\sigma|\left|\boldsymbol{u}_{K}^{n}-\boldsymbol{u}_{\sigma}^{n}\right| .
\end{gathered}
$$

We have by the Hölder inequality

$$
\begin{gathered}
\sum_{K \in \mathcal{T}} h|\sigma|\left|\boldsymbol{u}_{K}^{n}\right| \leq c\left(\sum_{\sigma \in \mathcal{T}} h|\sigma|\left|\boldsymbol{u}_{K}^{n}\right|^{6}\right)^{1 / 6} \leq c\left[\left(\sum_{K \in \mathcal{T}}\left\|\boldsymbol{u}^{n}-\boldsymbol{u}_{K}^{n}\right\|_{L^{6}\left(K ; \mathbb{R}^{3}\right)}^{6}\right)^{1 / 6}\right. \\
\left.+\left(\sum_{K \in \mathcal{T}}\left\|\boldsymbol{u}^{n}\right\|_{L^{6}\left(K ; \mathbb{R}^{3}\right)}^{6}\right)^{1 / 6}\right] \leq c\left(\sum_{K \in \mathcal{T}}\left\|\nabla \boldsymbol{u}_{n}\right\|_{L^{2}\left(K ; \mathbb{R}^{9}\right)}^{2}\right)^{1 / 2}, \\
\sum_{K \in \mathcal{T}} \sum_{\sigma \in \mathcal{E}(K)} h\left|\sigma \| \boldsymbol{u}_{K}^{n}-\boldsymbol{u}_{\sigma}^{n}\right| \leq c\left[\left(\sum_{K \in \mathcal{T}}\left\|\boldsymbol{u}^{n}-\boldsymbol{u}_{K}^{n}\right\|_{L^{2}\left(K ; \mathbb{R}^{3}\right)}^{2}\right)^{1 / 2}\right. \\
\left.+\left(\sum_{K \in \mathcal{T}} \sum_{\sigma \in \mathcal{E}(K)}\left\|\boldsymbol{u}^{n}-\boldsymbol{u}_{\sigma}^{n}\right\|_{L^{2}\left(K ; \mathbb{R}^{3}\right)}^{2}\right)^{1 / 2}\right] \leq h c\left(\sum_{K \in \mathcal{T}}\left\|\nabla \boldsymbol{u}_{n}\right\|_{L^{2}\left(K ; \mathbb{R}^{9}\right)}^{2}\right)^{1 / 2},
\end{gathered}
$$

where we have used $(2.53)_{p=2},(2.51+2.52)_{p=2}$. Consequently, we may use (4.4) to conclude

$$
\left|\mathcal{R}_{3,2}\right| \leq h c\left(M_{0}, E_{0}, \bar{r},\|\nabla r, \boldsymbol{V}, \nabla \boldsymbol{V}\|_{L^{\infty}\left(Q_{T} ; \mathbb{R}^{15}\right)}\right) .
$$

Finally, we replace in $\mathcal{T}_{3,2} \boldsymbol{V}_{\sigma}^{n}-\boldsymbol{V}_{h, K}^{n}$ by $\boldsymbol{V}_{h, 0, \sigma}^{n}-\boldsymbol{V}_{h, 0, K}^{n}$. We get

$$
\begin{gathered}
\mathcal{T}_{3,2}=\mathcal{T}_{3,3}+\mathcal{R}_{3,3}, \quad \mathcal{R}_{3,3}=\Delta t \sum_{n=1}^{m} \mathcal{R}_{3,3}^{n}, \\
\mathcal{T}_{3,3}=\Delta t \sum_{n=1}^{m} \sum_{K \in \mathcal{T}} \sum_{\sigma \in \mathcal{E}(K)}|\sigma| \hat{r}_{\sigma}^{n, \text { up }}\left[\hat{\boldsymbol{V}}_{h, 0, \sigma}^{n \text {,up }} \cdot \mathbf{n}_{\sigma, K}\right]\left(\boldsymbol{V}_{h, 0, \sigma}^{n}-\boldsymbol{V}_{h, 0, K}^{n}\right) \cdot\left(\hat{\boldsymbol{V}}_{h, 0, \sigma}^{n, \text { up }}-\hat{\boldsymbol{u}}_{\sigma}^{n, \text { up }}\right),
\end{gathered}
$$

and

$$
\mathcal{R}_{3,3}^{n}=\sum_{K \in \mathcal{T}} \sum_{\sigma \in \mathcal{E}(K)}|\sigma|\left(r_{K}^{n}-\hat{r}_{\sigma}^{n, \text { up }}\right) \boldsymbol{V}_{h, 0, K}^{n} \cdot \mathbf{n}_{\sigma, K}\left(\left[\boldsymbol{V}^{n}-\boldsymbol{V}_{h, 0}\right]_{\sigma}^{n}-\left[\boldsymbol{V}_{h}^{n}-\boldsymbol{V}_{h, 0}^{n}\right]_{K}\right) \cdot\left(\hat{\boldsymbol{V}}_{h, 0, \sigma}^{n, \text { up }}-\hat{\boldsymbol{u}}_{\sigma}^{n, \text { up }}\right),
$$

committing error

$$
\left|\mathcal{R}_{3,3}^{n}\right|=\leq h c\left(M_{0}, E_{0}, \bar{r},\|\nabla r, \boldsymbol{V}, \nabla \boldsymbol{V}\|_{L^{\infty}\left(Q_{T} ; \mathbb{R}^{15}\right)}\right)
$$


as in the previous step.

Step 4: Terms $\mathcal{T}_{4}$ We write

$$
\begin{gathered}
\mathcal{T}_{4}=\mathcal{T}_{4,1}+\mathcal{R}_{4,1}, \quad \mathcal{T}_{4,1}=-\int_{\Omega_{h}} \nabla p\left(r^{n}\right) \cdot \mathbf{V}^{n} \mathrm{~d} x, \\
\mathcal{R}_{4,1}=\int_{\Omega_{h}} \nabla p\left(r^{n}\right) \cdot\left(\mathbf{V}^{n}-\boldsymbol{V}_{h, 0}^{n}\right) \mathrm{d} x ;
\end{gathered}
$$

whence

$$
\left|\mathcal{R}_{4,1}\right| \leq h c\left(\bar{r},\left|p^{\prime}\right|_{C[\underline{r}, \bar{r}]},\|\nabla r\|_{L^{\infty}\left(Q_{T} ; \mathbb{R}^{3}\right)}\right),
$$

by virtue of $(2.39) s_{s=1},(2.47 \sqrt[2.48)]{ }$.

Next, employing the integration by parts

$$
\begin{gathered}
\mathcal{T}_{4,2}=\mathcal{T}_{4,2}+\mathcal{R}_{4,2}, \quad \mathcal{T}_{4,2}=\int_{\Omega_{h}} p\left(r^{n}\right) \operatorname{div} \mathbf{V}^{n} \mathrm{~d} x, \\
\mathcal{R}_{4,2}=-\sum_{K \in \mathcal{T}} \sum_{\sigma \in \mathcal{E}(K), \sigma \in \partial \Omega_{h}} \int_{\sigma} p\left(r^{n}\right) \mathbf{V}^{n} \cdot \mathbf{n}_{\sigma, K} \mathrm{~d} S=-\sum_{K \in \mathcal{T}} \sum_{\sigma \in \mathcal{E}(K), \sigma \in \partial \Omega_{h}} \int_{\sigma} p\left(r^{n}\right)\left(\mathbf{V}^{n}-\boldsymbol{V}_{h, 0, \sigma}^{n}\right) \cdot \mathbf{n}_{\sigma, K} \mathrm{~d} S .
\end{gathered}
$$

Writing

$$
\mathbf{V}^{n}-\boldsymbol{V}_{h, 0, \sigma}^{n}=\mathbf{V}^{n}-\boldsymbol{V}_{h}^{n}+\mathbf{V}_{h}^{n}-\boldsymbol{V}_{h, \sigma}^{n}+\left[\boldsymbol{V}_{h}^{n}-\boldsymbol{V}_{h, 0}^{n}\right]_{\sigma},
$$

we deduce by using $(2.39)_{s=1},(2.40)_{s=1},(2.52)_{p=\infty}$, (2.47), (2.48),

$$
\left\|\mathbf{V}^{n}-\boldsymbol{V}_{h, 0, \sigma}^{n}\right\|_{L^{\infty}\left(K ; \mathbb{R}^{3}\right)} \lesssim h\left\|\nabla \boldsymbol{V}^{n}\right\|_{L^{\infty}\left(K ; \mathbb{R}^{3}\right)}, \sigma \in K .
$$

Now, we employ the fact that

$$
\sum_{K \in \mathcal{T}} \sum_{\sigma \in \mathcal{E}(K), \sigma \in \partial \Omega_{h}} \int_{\sigma} \mathrm{d} S \approx 1
$$

whence

$$
\left|\mathcal{R}_{4,2}\right| \leq h c\left(\bar{r},|p|_{C[r, \bar{r}]},\|\nabla \mathbf{V}\|_{L^{\infty}\left(Q_{T} ; \mathbb{R}^{9}\right)}\right)
$$

Finally,

$$
\mathcal{T}_{4,2}=\mathcal{T}_{4,3}+\mathcal{R}_{4,3}, \quad \mathcal{T}_{4,3}=\int_{\Omega_{h}} p\left(\hat{r}^{n}\right) \operatorname{div} \mathbf{V}^{n} \mathrm{~d} x, \quad \mathcal{R}_{4,3}=\int_{\Omega_{h}}\left(p\left(r^{n}\right)-p\left(\hat{r}^{n}\right)\right) \operatorname{div} \mathbf{V}^{n} \mathrm{~d} x
$$

whence

$$
\left|\mathcal{R}_{4,3}\right| \leq h c\left(\left|p^{\prime}\right|_{C[\underline{r}, \bar{r}]},\|(\nabla r, \nabla \mathbf{V})\|_{L^{\infty}\left(Q_{T} ; \mathbb{R}^{12}\right)}\right) .
$$

Step 5: $\operatorname{Term} \mathcal{T}_{6}$ We decompose $\mathcal{T}_{6}$ as

$$
\begin{aligned}
& \mathcal{T}_{6}=\mathcal{T}_{6,1}+\mathcal{R}_{6,1}, \text { with } \mathcal{T}_{6,1}=-\Delta t \sum_{n=1}^{m} \sum_{K \in \mathcal{T}} \int_{K} p^{\prime}\left(\hat{r}^{n}\right) \boldsymbol{u}^{n} \cdot \nabla r^{n} \mathrm{~d} x \\
& \mathcal{R}_{6,1}=\Delta t \sum_{n=1}^{m} \sum_{K \in \mathcal{T}} \int_{K}\left(p^{\prime}\left(\hat{r}^{n}\right)-p^{\prime}\left(r^{n}\right)\right) \cdot \boldsymbol{u}^{n} \cdot \nabla r^{n} \mathrm{~d} x
\end{aligned}
$$

Consequently, by the Taylor formula, Hölder inequality and estimate (4.5),

$$
\left|\mathcal{R}_{6,1}\right| \leq h c\left(M_{0}, E_{0}, \underline{r}, \bar{r},\left|p^{\prime}\right|_{C^{1}([\underline{r}, \bar{r}])},\|\nabla r\|_{L^{\infty}\left(Q_{T} ; \mathbb{R}^{3}\right)}\right) .
$$

Gathering the formulae (7.7), (17.12), (77.17), (17.21), (77.23) and estimates for the residual terms (7.8), (7.9 (7.13), (7.14 (7.18), (7.19), (7.20), (7.22), (7.24) concludes the proof of Lemma 7.1 . 


\section{A Gronwall inequality}

In this Section we put together the relative energy inequality (6.1) and the identity (7.1) derived in the previous section. The final inequality resulting from this manipulation is formulated in the following lemma.

Lemma 8.1. Let $\left(\varrho^{n}, \boldsymbol{u}^{n}\right)$ be a solution of the discrete problem (3.5 3.7) with the pressure satisfying (1.4), where $\gamma \geq 3 / 2$. Then there exists a positive number

$$
\begin{gathered}
c=c\left(M_{0}, E_{0}, \underline{r}, \bar{r},\left|p^{\prime}\right|_{C^{1}[\underline{r}, \bar{r}]},\left\|\left(\partial_{t} r, \nabla r, \boldsymbol{V}, \partial_{t} \boldsymbol{V}, \nabla \boldsymbol{V}, \nabla^{2} \boldsymbol{V}\right)\right\|_{L^{\infty}\left(Q_{T} ; \mathbb{R}^{45}\right)},\right. \\
\left.\left\|\partial_{t}^{2} r\right\|_{L^{1}\left(0, T ; L^{\prime}(\Omega)\right)},\left\|\partial_{t} \nabla r\right\|_{L^{2}\left(0, T ; L^{6 \gamma / 5 \gamma-6}\left(\Omega ; \mathbb{R}^{3}\right)\right)},\left\|\partial_{t}^{2} \boldsymbol{V}, \partial_{t} \nabla \boldsymbol{V}\right\|_{L^{2}\left(0, T ; L^{6 / 5}\left(\Omega ; \mathbb{R}^{12}\right)\right)}\right),
\end{gathered}
$$

such that for all $m=1, \ldots, N$, there holds:

$$
\begin{gathered}
\mathcal{E}\left(\varrho^{m}, \boldsymbol{u}^{m} \mid \hat{r}^{m}, \hat{\boldsymbol{V}}_{h, 0}^{m}\right)+\Delta t \frac{\mu}{2} \sum_{n=1}^{m} \sum_{K \in \mathcal{T}} \int_{K}\left|\nabla_{x}\left(u^{n}-\mathbf{V}_{h, 0}^{n}\right)\right|^{2} \mathrm{dx} \\
\leq c\left[h^{a}+\sqrt{\Delta t}+\mathcal{E}\left(\varrho^{0}, \boldsymbol{u}^{0} \mid \hat{r}(0), \hat{\boldsymbol{V}}_{h, 0}(0)\right)\right]+c \Delta t \sum_{n=1}^{m} \mathcal{E}\left(\varrho^{n}, \boldsymbol{u}^{n} \mid \hat{r}^{n}, \hat{\boldsymbol{V}}_{h, 0}^{n}\right),
\end{gathered}
$$

with any couple $(r, \mathbf{V})$ belonging to (2.25) and satisfying the continuity equation (1.1) on $(0, T) \times \mathbb{R}^{3}$ and momentum equation (1.2) with boundary conditions (1.5) on $(0, T) \times \Omega$ in the classical sense, where a is defined in (6.3) and $\mathcal{E}$ is given in (4.8).

Proof. We observe that

$$
S_{6}-\mathcal{S}_{6}=\Delta t \sum_{n=1}^{m} \int_{\Omega_{h}} p^{\prime}\left(\hat{r}^{n}\right) \frac{\hat{r}^{n}-\varrho^{n}}{\hat{r}^{n}} \mathbf{V}^{n} \cdot \nabla r^{n} \mathrm{~d} x+\Delta t \sum_{n=1}^{m} \int_{\Omega_{h}} p^{\prime}\left(\hat{r}^{n}\right) \frac{\hat{r}^{n}-\varrho^{n}}{\hat{r}^{n}}\left(\mathbf{u}^{n}-\mathbf{V}^{n}\right) \cdot \nabla r^{n} \mathrm{~d} x .
$$

Gathering the formulae (6.1) and (6.2), one gets

$$
\mathcal{E}\left(\varrho^{m}, \boldsymbol{u}^{m} \mid \hat{r}^{m}, \hat{\boldsymbol{V}}_{h, 0}^{m}\right)-\mathcal{E}\left(\varrho^{0}, \boldsymbol{u}^{0} \mid \hat{r}(0), \hat{\boldsymbol{V}}_{h, 0}(0)\right)+\mu \Delta t \sum_{n=1}^{m} \sum_{K \in \mathcal{T}}\left|\nabla\left(\boldsymbol{u}^{n}-\boldsymbol{V}_{0, h}^{n}\right)\right|_{L^{2}\left(K ; \mathbb{R}^{3}\right)}^{2} \leq \sum_{i=1}^{4} \mathcal{P}_{i}+\mathcal{Q}
$$

where

$$
\begin{aligned}
& \mathcal{P}_{1}=\Delta t \sum_{n=1}^{m} \sum_{K \in \mathcal{T}}|K|\left(\varrho_{K}^{n-1}-r_{K}^{n-1}\right) \frac{\boldsymbol{V}_{h, 0, K}^{n}-\boldsymbol{V}_{h, 0, K}^{n-1}}{\Delta t} \cdot\left(\boldsymbol{V}_{h, 0, K}^{n}-\boldsymbol{u}_{K}^{n}\right), \\
& \mathcal{P}_{2}=\Delta t \sum_{n=1}^{m} \sum_{K \in \mathcal{T}} \sum_{\sigma=K \mid L \in \mathcal{E}_{K}}|\sigma|\left(\varrho_{\sigma}^{n, \text { up }}-\hat{r}_{\sigma}^{n, \text { up }}\right)\left(\hat{\boldsymbol{V}}_{h, 0, \sigma}^{n, \text { up }}-\hat{\boldsymbol{u}}_{\sigma}^{n, \text { up }}\right) \cdot\left(\boldsymbol{V}_{h, 0, \sigma}^{n}-\boldsymbol{V}_{h, 0, K}^{n}\right) \boldsymbol{V}_{h, 0, \sigma}^{n, \text { up }} \cdot \boldsymbol{n}_{\sigma, K}, \\
& \mathcal{P}_{3}=-\Delta t \sum_{n=1}^{m} \int_{\Omega_{h}}\left(p\left(\varrho^{n}\right)-p^{\prime}\left(\hat{r}^{n}\right)\left(\varrho^{n}-\hat{r}^{n}\right)-p\left(\hat{r}^{n}\right)\right) \operatorname{div} \mathbf{V}^{n}, \\
& \mathcal{P}_{4}=\Delta t \sum_{n=1}^{m} \sum_{K \in \mathcal{T}} \int_{K} p^{\prime}\left(\hat{r}^{n}\right) \frac{\hat{r}^{n}-\varrho^{n}}{\hat{r}^{n}}\left(\mathbf{u}^{n}-\mathbf{V}^{n}\right) \cdot \nabla r^{n} \mathrm{~d} x \\
& \mathcal{Q}=\mathcal{R}_{h, \Delta t}^{m}+R_{h, \Delta t}^{m}+G^{m} .
\end{aligned}
$$

Now, we estimate conveniently the terms $\mathcal{P}_{i}, i=1, \ldots, 4$ in four steps.

Step 1: Term $\mathcal{P}_{1}$. We estimate the $L^{\infty}$ norm of $\frac{\boldsymbol{V}_{h, 0, K}^{n}-\boldsymbol{V}_{h, 0, K}^{n-1}}{\Delta t}$ by $L^{\infty}$ norm of $\partial_{t} \mathbf{V}$ in the same manner as in (6.5). According to Lemma [7.2, $|\varrho-r|^{\gamma} 1_{\left.R_{+} \backslash \underline{r} / 2,2 \bar{r}\right]}(\varrho) \leq c(p) E^{p}(\varrho \mid r)$, with any $p \geq 1$; in particular,

$$
|\varrho-r|^{6 / 5} 1_{R_{+} \backslash[\underline{r} / 2,2 \bar{r}]}(\varrho) \leq c E(\varrho \mid r)
$$


provided $\gamma \geq 6 / 5$.

We get by using the Hölder inequality,

$$
\begin{gathered}
\left|\sum_{K \in \mathcal{T}}\right| K\left|\left(\varrho_{K}^{n-1}-r_{K}^{n-1}\right) \frac{\boldsymbol{V}_{h, 0, K}^{n}-\boldsymbol{V}_{h, 0, K}^{n-1}}{\Delta t} \cdot\left(\boldsymbol{V}_{h, K}^{n}-\boldsymbol{u}_{K}^{n}\right)\right| \leq c\left\|\partial_{t} \boldsymbol{V}\right\|_{L^{\infty}\left(Q_{T} ; \mathbb{R}^{3}\right)} \times \\
{\left[\left(\sum_{K \in \mathcal{T}}|K|\left|\varrho_{K}^{n-1}-r_{K}^{n-1}\right|^{2} 1_{[\underline{r} / 2,2 \bar{r}]}\left(\varrho_{K}\right)\right)^{1 / 2}+\left(\sum_{K \in \mathcal{T}}\left|K \| \varrho_{K}^{n-1}-r_{K}^{n-1}\right|{ }^{6 / 5} 1_{R_{+} \backslash[\underline{r} / 2,2 \bar{r}]}\left(\varrho_{K}\right)\right)^{5 / 6}\right] \times} \\
\left(\sum_{K \in \mathcal{T}}|K|\left|\boldsymbol{V}_{h, 0, K}^{n}-\boldsymbol{u}_{K}^{n}\right|^{6}\right)^{1 / 6} \leq c\left(\left\|\left(\partial_{t} \boldsymbol{V}\right)\right\|_{L^{\infty}\left(Q_{T} ; \mathbb{R}^{3}\right)}\right)\left(\mathcal{E}^{1 / 2}\left(\varrho^{n-1}, \hat{\boldsymbol{u}}^{n-1} \mid \hat{r}^{n-1}, \hat{\boldsymbol{V}}_{h, 0}^{n-1}\right)\right. \\
\left.+\mathcal{E}^{5 / 6}\left(\varrho^{n-1}, \hat{\boldsymbol{u}}^{n-1} \mid \hat{r}^{n-1}, \hat{\boldsymbol{V}}_{h, 0}^{n-1}\right)\right)\left(\sum_{K \in \mathcal{T}}\left\|\boldsymbol{V}_{h, 0, K}^{n}-\boldsymbol{u}_{K}^{n}\right\|_{L^{6}\left(K ; \mathbb{R}^{3}\right)}^{6}\right)^{1 / 6},
\end{gathered}
$$

where we have used (8.2) and estimate (4.8) to obtain the last line. Now, we write $\boldsymbol{V}_{h, 0, K}^{n}-\boldsymbol{u}_{K}^{n}=$ $\left(\left[\boldsymbol{V}_{h, 0}^{n}-\boldsymbol{u}^{n}\right]_{K}-\left(\boldsymbol{V}_{h, 0}^{n}-\boldsymbol{u}^{n}\right)\right)+\left(\boldsymbol{V}_{h, 0}^{n}-\boldsymbol{u}^{n}\right)$ and use the Minkowski inequality together with formulas (2.53), (2.42) to get

$$
\left(\sum_{K \in \mathcal{T}}\left\|\boldsymbol{V}_{h, 0, K}^{n}-\boldsymbol{u}_{K}^{n}\right\|_{L^{6}\left(K ; \mathbb{R}^{3}\right)}^{6}\right)^{1 / 6} \leq\left(\sum_{K \in \mathcal{T}}\left\|\nabla\left(\boldsymbol{V}_{h, 0}^{n}-\boldsymbol{u}^{n}\right)\right\|_{L^{2}\left(K ; \mathbb{R}^{3}\right)}^{2}\right)^{1 / 2} .
$$

Finally, employing Young's inequality, and estimate (4.8), we arrive at

$$
\begin{aligned}
& \left|\mathcal{P}_{1}\right| \leq c\left(\delta, M_{0}, E_{0}, \underline{r}, \bar{r},\left\|\left(\boldsymbol{V}, \nabla \boldsymbol{V}, \partial_{t} \boldsymbol{V}\right)\right\|_{L^{\infty}\left(Q_{T}, \mathbb{R}^{15}\right)}\right) \\
& \quad \times\left(\Delta t \mathcal{E}\left(\varrho^{0}, \hat{\boldsymbol{u}}^{0} \mid \hat{r}^{0}, \hat{\boldsymbol{V}}_{h, 0}^{0}\right)+\Delta t \sum_{n=1}^{m} \mathcal{E}\left(\varrho^{n}, \hat{\boldsymbol{u}}^{n} \mid \hat{r}^{n}, \hat{\boldsymbol{V}}_{h, 0}^{n}\right)\right)+\delta \Delta t \sum_{n=1}^{m} \sum_{K \in \mathcal{T}}\left\|\nabla\left(\boldsymbol{V}_{h, 0}^{n}-\boldsymbol{u}^{n}\right)\right\|_{L^{2}\left(K ; \mathbb{R}^{3}\right)}^{2}
\end{aligned}
$$

with any $\delta>0$.

Step 2: $\operatorname{Term} \mathcal{P}_{2}$. We rewrite $\boldsymbol{V}_{h_{0}, \sigma}^{n}-\boldsymbol{V}_{h_{0}, K}^{n}=\boldsymbol{V}_{h, \sigma}^{n}-\boldsymbol{V}_{h, K}^{n}+\left[\boldsymbol{V}_{h, 0}^{n}-\boldsymbol{V}_{h}^{n}\right]_{\sigma}+\left[\boldsymbol{V}_{h, 0}^{n}-\boldsymbol{V}_{h}^{n}\right]_{K}$ and estimate the $L^{\infty}$ norm of this expression by $h\|\nabla \boldsymbol{V}\|_{L^{\infty}\left(Q_{T} ; \mathbb{R}^{9}\right)}$ by virtue of (2.47 2.48), (2.51 2.52), $(2.40)_{s=1}$. Now we write $\mathcal{P}_{2}=\Delta t \sum_{n=1}^{m} \mathcal{P}_{2}^{n}$ where Lemma 7.2 and the Hölder inequality yield, similarly as in the previous step,

$$
\begin{aligned}
\left|\mathcal{P}_{2}^{n}\right| \leq & c\left(\underline{r}, \bar{r},\|\nabla \boldsymbol{V}\|_{L^{\infty}\left(Q_{T} ; \mathbb{R}^{9}\right)}\right) \times \\
& \sum_{K \in \mathcal{T}} \sum_{\sigma \in \mathcal{E}(K)}|\sigma| h\left(E^{1 / 2}\left(\varrho_{\sigma}^{n, \text { up }} \mid \hat{r}_{\sigma}^{n, \text { up }}\right)+E^{2 / 3}\left(\varrho_{\sigma}^{n, \text { up }} \mid \hat{r}_{\sigma}^{n, \text { up }}\right)\left|\hat{\boldsymbol{V}}_{h, 0, \sigma}^{n, \text { up }}\right|\left|\hat{\boldsymbol{V}}_{h, 0, \sigma}^{n, \text { up }}-\hat{\boldsymbol{u}}_{\sigma}^{n, \text { up }}\right|\right. \\
& \leq c\left(\underline{r}, \bar{r},\|(\boldsymbol{V}, \nabla \boldsymbol{V})\|_{L^{\infty}\left(Q_{T} ; \mathbb{R}^{12}\right)}\right)\left[\left(\sum_{K \in \mathcal{T}} \sum_{\sigma \in \mathcal{E}(K)}|\sigma| h\left(E\left(\varrho_{\sigma}^{n, \text { up }} \mid \hat{r}_{\sigma}^{n, \text { up }}\right)\right)^{1 / 2}\right.\right. \\
& \left.+\left(\sum_{K \in \mathcal{T}} \sum_{\sigma \in \mathcal{E}(K)}|\sigma| h E\left(\varrho_{\sigma}^{n, \text { up }} \mid \hat{r}_{\sigma}^{n, \text { up }}\right)\right)^{2 / 3}\right] \times\left(\sum_{K \in \mathcal{T}} \sum_{\sigma \in \mathcal{E}(K)}|\sigma| h\left|\hat{\boldsymbol{V}}_{h, 0, \sigma}^{n, \text { up }}-\hat{\boldsymbol{u}}_{\sigma}^{n, \text { up }}\right|^{6}\right)^{1 / 6},
\end{aligned}
$$

provided $\gamma \geq 3 / 2$. Next, we observe that the contribution of the face $\sigma=K \mid L$ to the sums $\sum_{K \in \mathcal{T}}$ $\sum_{\sigma \in \mathcal{E}(K)}|\sigma| h E\left(\varrho_{\sigma}^{n, \text { up }} \mid \hat{r}_{\sigma}^{n, \text { up }}\right)$ and $\sum_{K \in \mathcal{T}} \sum_{\sigma \in \mathcal{E}(K)}|\sigma| h\left|\hat{\boldsymbol{V}}_{h, 0, \sigma}^{n \text {,up }}-\hat{\boldsymbol{u}}_{\sigma}^{n, \text { up }}\right|^{6}$ is less or equal than $2|\sigma| h\left(E\left(\varrho_{K}^{n} \mid \hat{r}_{K}^{n}\right)+\right.$ $\left.E\left(\varrho_{L}^{n} \mid \hat{r}_{L}^{n}\right)\right)$, and than $2|\sigma| h\left(\left|\boldsymbol{V}_{h, 0, K}^{n}-\boldsymbol{u}_{K}^{n}\right|^{6}+\left|\boldsymbol{V}_{h, 0, L}^{n}-\boldsymbol{u}_{L}^{n}\right|^{6}\right)$, respectively. Consequently, we get by the same reasoning as in the previous step, under assumption $\gamma \geq 3 / 2$,

$\left|\mathcal{P}_{2}\right| \leq c\left(\delta, M_{0}, E_{0}, \underline{r}, \bar{r},\|(\boldsymbol{V}, \nabla \boldsymbol{V})\|_{L^{\infty}\left(Q_{T} ; \mathbb{R}^{12}\right)}\right) \Delta t \sum_{n=1}^{m} \mathcal{E}\left(\varrho^{n}, \hat{\boldsymbol{u}}^{n} \mid \hat{r}^{n}, \hat{\boldsymbol{V}}_{h, 0}^{n}\right)+\delta \Delta t \sum_{n=1}^{m} \sum_{K \in \mathcal{T}}\left\|\nabla\left(\boldsymbol{V}_{h, 0}^{n}-\boldsymbol{u}^{n}\right)\right\|_{L^{2}\left(K ; \mathbb{R}^{3}\right)}^{2}$ 
Step 3: Term $\mathcal{P}_{3}$. We realize that

$$
p\left(\varrho_{K}^{n}\right)-p^{\prime}\left(r_{K}^{n}\right)\left(\varrho_{K}^{n}-r_{K}^{n}\right)-p\left(r_{K}^{n}\right) \leq c(\underline{r}, \bar{r}) E\left(\varrho_{K} \mid r_{K}\right),
$$

by virtue of Lemma 7.2 in combination with assumption (1.4). Consequently,

$$
\left|\mathcal{P}_{3}\right| \leq c\|\operatorname{div} \boldsymbol{V}\|_{L^{\infty}\left(Q_{T}\right)} \Delta t \sum_{n=1}^{m} \mathcal{E}\left(\varrho^{n}, \hat{\boldsymbol{u}}^{n} \mid \hat{r}^{n}, \hat{\boldsymbol{V}}_{h, 0}^{n}\right) .
$$

Step 4:Term $\mathcal{P}_{4}$. We write $\boldsymbol{u}^{n}-\boldsymbol{V}^{n}$ as the sum $\left(\boldsymbol{u}^{n}-\boldsymbol{V}_{h, 0}^{n}\right)+\left(\boldsymbol{V}_{h, 0}^{n}-\boldsymbol{V}^{n}\right)$ accordingly splitting $\mathcal{P}_{4}$ into two terms

$\Delta t \sum_{n=1}^{m} \sum_{K \in \mathcal{T}} \int_{K} p^{\prime}\left(\hat{r}^{n}\right) \frac{\hat{r}^{n}-\varrho^{n}}{\hat{r}^{n}}\left(\mathbf{u}^{n}-\boldsymbol{V}_{h, 0}^{n}\right) \cdot \nabla r^{n} \mathrm{~d} x \quad$ and $\quad \Delta t \sum_{n=1}^{m} \sum_{K \in \mathcal{T}} \int_{K} p^{\prime}\left(\hat{r}^{n}\right) \frac{\hat{r}^{n}-\varrho^{n}}{\hat{r}^{n}}\left(\boldsymbol{V}_{h, 0}^{n}-\mathbf{V}^{n}\right) \cdot \nabla r^{n} \mathrm{~d} x$.

Reasoning similarly as in Step 2, we get

$$
\begin{aligned}
\left|\mathcal{P}_{4}\right| & \leq h^{2} c\left(\delta, M_{0}, E_{0}, \underline{r}, \bar{r},\left|p^{\prime}\right|_{C([\underline{r}, \bar{r}])}\|(\nabla r, \nabla \boldsymbol{V})\|_{L^{\infty}\left(\Omega ; \mathbb{R}^{9}\right)}\right) \\
& +c\left(\delta,\left\|\underline{r}, \bar{r},\left|p^{\prime}\right|_{C([\underline{r}, \bar{r}]}\right\| \nabla r \|_{L^{\infty}\left(\Omega ; \mathbb{R}^{3}\right)}\right) \Delta t \sum_{n=1}^{m} \mathcal{E}\left(\varrho^{n}, \hat{\boldsymbol{u}}^{n} \mid \hat{r}^{n}, \hat{\boldsymbol{V}}_{h, 0}^{n}\right)+\delta \Delta t \sum_{n=1}^{m} \sum_{K \in \mathcal{T}}\left\|\nabla\left(\boldsymbol{V}_{h, 0}^{n}-\boldsymbol{u}^{n}\right)\right\|_{L^{2}\left(K ; \mathbb{R}^{3}\right)}^{2} .
\end{aligned}
$$

Gathering the formulae (8.1) and (8.3)-(8.6) with $\delta$ sufficiently small (with respect to $\mu$ ), we conclude the proof of Lemma 8.1 ,

\section{End of the proof of the error estimate (Theorem 3.1)}

Finally, Lemma 8.1 in combination with the bound (4.8) yields

$$
\mathcal{E}\left(\varrho^{m}, \hat{\boldsymbol{u}}^{m} \mid \hat{r}^{m}, \hat{\boldsymbol{V}}_{h, 0}^{m}\right) \leq c\left[h^{A}+\sqrt{\Delta t}+\Delta t+\mathcal{E}\left(\varrho^{0}, \hat{\boldsymbol{u}}^{0} \mid \hat{r}(0), \hat{\boldsymbol{V}}_{h, 0}(0)\right)\right]+c \Delta t \sum_{n=1}^{m-1} \mathcal{E}\left(\varrho^{n}, \hat{\boldsymbol{u}}^{n} \mid \hat{r}^{n}, \hat{\boldsymbol{V}}_{h, 0}^{n}\right) ;
$$

whence by the discrete standard version of the Gronwall lemma one gets at the first step

$$
\mathcal{E}\left(\varrho^{m}, \hat{\boldsymbol{u}}^{m} \mid \hat{r}^{m}, \hat{\boldsymbol{V}}_{h, 0}^{m}\right) \leq c\left[h^{a}+\sqrt{\Delta t}+\mathcal{E}\left(\varrho^{0}, \hat{\boldsymbol{u}}^{0} \mid \hat{r}(0), \hat{\boldsymbol{V}}_{h, 0}(0)\right)\right] .
$$

Going with this information back to Lemma 8.1, one gets finally

$$
\mathcal{E}\left(\varrho^{m}, \hat{\boldsymbol{u}}^{m} \mid \hat{r}^{m}, \hat{\boldsymbol{V}}_{h, 0}^{m}\right)+\Delta t \frac{\mu}{2} \sum_{n=1}^{m} \sum_{K \in \mathcal{T}} \int_{K}\left|\nabla_{x}\left(\mathbf{u}^{n}-\mathbf{V}_{h, 0}^{n}\right)\right|^{2} \mathrm{dx} \leq c\left[h^{a}+\sqrt{\Delta t}+\mathcal{E}\left(\varrho^{0}, \hat{\boldsymbol{u}}^{0} \mid \hat{r}(0), \hat{\boldsymbol{V}}_{h, 0}(0)\right)\right] .
$$

Now, we write

$$
\varrho_{K}^{n}\left(\boldsymbol{u}_{K}^{n}-\mathbf{V}_{h, 0, K}^{n}\right)^{2}=\varrho_{K}^{n}\left(\boldsymbol{u}_{K}^{n}-\mathbf{V}^{n}\right)^{2}+2 \varrho_{K}^{n} \mathbf{V}^{n}\left(\boldsymbol{u}_{K}^{n}-\mathbf{V}_{h, 0, K}^{n}\right)+\varrho_{K}^{n}\left(\mathbf{V}^{n}-\mathbf{V}_{h, 0, K}^{n}\right)^{2},
$$

where

$$
\begin{aligned}
\| \mathbf{V}^{n}- & \mathbf{V}_{h, 0, K}^{n}\left\|_{L^{\infty}\left(K ; \mathbb{R}^{3}\right)} \lesssim\right\| \mathbf{V}^{n}-\mathbf{V}_{h}^{n}\left\|_{L^{\infty}\left(K ; \mathbb{R}^{3}\right)}+\right\| \mathbf{V}_{h}^{n}-\mathbf{V}_{h, K}^{n}\left\|_{L^{\infty}\left(K ; \mathbb{R}^{3}\right)}+\right\|\left[\mathbf{V}_{h}^{n}-\mathbf{V}_{h, 0}^{n}\right]_{K} \|_{L^{\infty}\left(K ; \mathbb{R}^{3}\right)} \\
& \lesssim h\left(\left\|\nabla_{x} \mathbf{V}^{n}\right\|_{L^{\infty}\left(K ; \mathbb{R}^{9}\right)}+\left\|\nabla_{x} \mathbf{V}_{h}^{n}\right\|_{L^{\infty}\left(K ; \mathbb{R}^{9}\right)}+\left\|\mathbf{V}_{h}^{n}-\mathbf{V}_{h, 0}^{n}\right\|_{L^{\infty}\left(K ; \mathbb{R}^{3}\right)} \lesssim h\left\|\nabla \mathbf{V}^{n}\right\|_{L^{\infty}\left(K ; \mathbb{R}^{9}\right)} .\right.
\end{aligned}
$$

In the above calculation we have employed formula (2.39) to estimate the first term, estimates (2.51) $s=1$, (2.40) $s=1$ to estimate the second term, and formulas (2.47) and (2.48) for $K \cap \partial \Omega_{h}=\emptyset$ and $K \cap \partial \Omega_{h} \neq \emptyset$, respectively, to evaluate the last term. We conclude that

$$
\sum_{K \in \mathcal{T}} \frac{1}{2}|K|\left(\varrho_{K}^{m}\left|\boldsymbol{u}_{K}^{m}-\boldsymbol{V}_{h, 0, K}^{m}\right|^{2}-\varrho_{K}^{0}\left|\boldsymbol{u}_{K}^{0}-\boldsymbol{V}_{h, 0, K}^{0}\right|^{2}\right)
$$




$$
\geq \int_{\Omega \cap \Omega_{h}} \varrho^{m}\left(\hat{\boldsymbol{u}}^{m}-\mathbf{V}^{m}\right)^{2} \mathrm{~d} x-\int_{\Omega \cap \Omega_{h}} \varrho^{0}\left(\hat{\boldsymbol{u}}^{0}-\mathbf{V}^{0}\right)^{2} \mathrm{~d} x+L_{1},
$$

where

$$
\left|L_{1}\right| \lesssim h M_{0}\left\|\nabla_{x} \mathbf{V}\right\|_{L^{\infty}\left((0, T) \times \Omega ; \mathbb{R}^{9}\right)} .
$$

Similarly, we find with help of (4.8),

$$
\left\|E\left(\varrho_{K}^{n} \mid \hat{r}^{n}\right)-E\left(\varrho_{K}^{n}, r^{n}\right)\right\|_{L^{\infty}(K)} \leq h c\left(M_{0}, \underline{r}, \bar{r},|p|_{C^{1}[\underline{r}, \bar{r}]}\|\nabla r\|_{L^{\infty}\left(Q_{T} ; \mathbb{R}^{3}\right)}\right)
$$

whence

$$
\sum_{K \in \mathcal{T}}|K|\left(E\left(\varrho_{K}^{n} \mid \hat{r}^{n}\right)-E\left(\varrho_{K}^{0} \mid \hat{r}^{0}\right) \geq \int_{\Omega \cap \Omega_{h}} E\left(\varrho^{m} \mid r^{m}\right) \mathrm{d} x-\int_{\Omega \cap \Omega_{h}} E\left(\varrho^{0} \mid r^{0}\right) \mathrm{d} x+L_{2}\right.
$$

where

$$
\left|L_{2}\right| \leq h c\left(M_{0}, \underline{r}, \bar{r},|p|_{C^{1}[\underline{r}, \bar{r}]},\|\nabla r\|_{L^{\infty}\left(Q_{T} ; \mathbb{R}^{3}\right)}\right) .
$$

Finally, by virtue of $(2.47 \sqrt{2.48})$ and $(2.40) s_{s=2}$

$$
\left\|\nabla\left(\boldsymbol{V}_{h, 0}^{n}-\boldsymbol{V}^{n}\right)\right\|_{L^{2}\left(K ; \mathbb{R}^{3}\right)} \lesssim h\left\|\left(\nabla \boldsymbol{V}^{n}, \nabla^{2} \boldsymbol{V}^{n}\right)\right\|_{L^{\infty}\left(K ; \mathbb{R}^{12}\right)}
$$

whence

$$
\Delta t \sum_{n=1}^{m} \sum_{K \in \mathcal{T}} \int_{K}\left|\nabla_{x}\left(\mathbf{u}^{n}-\mathbf{V}_{h, 0}^{n}\right)\right|^{2} \mathrm{dx} \geq \Delta t \sum_{n=1}^{m} \int_{\Omega \cap \Omega_{h}}\left|\left(\nabla_{h} \mathbf{u}^{n}-\nabla_{x} \mathbf{V}^{n}\right)\right|^{2} \mathrm{dx}+L_{3}
$$

where

$$
\left|L_{3}\right| \leq h^{2} c\left(\left\|\left(\nabla \boldsymbol{V}^{n}, \nabla^{2} \boldsymbol{V}^{n}\right)\right\|_{L^{\infty}\left(K ; \mathbb{R}^{12}\right)}\right) .
$$

Theorem 3.1 is a direct consequence of estimate (9.1) and identities (9.2 9.4). Theorem 3.1 is thus proved.

\section{Concluding remarks}

In the convergence proofs one usually needs to complete the numerical scheme by stabilizing terms, so that the new numerical scheme reads

$$
\begin{gathered}
\sum_{K \in \mathcal{T}_{h}}|K| \frac{\varrho_{K}^{n}-\varrho_{K}^{n-1}}{\Delta t} \phi_{K}+\sum_{K \in \mathcal{T}_{h}} \sum_{\sigma \in \mathcal{E}(K)}|\sigma| \varrho_{\sigma}^{n, \text { up }}\left(\mathbf{u}_{\sigma}^{n} \cdot \mathbf{n}_{\sigma, K}\right) \phi_{K}+T_{c}(\phi)=0, \\
\text { for any } \phi \in Q_{h}\left(\Omega_{h}\right) \text { and } n=1, \ldots, N, \\
\sum_{K \in \mathcal{T}} \frac{|K|}{\Delta t}\left(\varrho_{K}^{n} \boldsymbol{u}_{K}^{n}-\varrho_{K}^{n-1} \boldsymbol{u}_{K}^{n-1}\right) \cdot \boldsymbol{v}_{K}+\sum_{K \in \mathcal{T}} \sum_{\sigma \in \mathcal{E}(K)}|\sigma| \varrho_{\sigma}^{n, \text { up }} \hat{\boldsymbol{u}}_{\sigma}^{n, \text { up }}\left[\boldsymbol{u}_{\sigma}^{n} \cdot \boldsymbol{n}_{\sigma, K}\right] \cdot \boldsymbol{v}_{K} \\
-\sum_{K \in \mathcal{T}} p\left(\varrho_{K}^{n}\right) \sum_{\sigma \in \mathcal{E}(K)}|\sigma| \boldsymbol{v}_{\sigma} \cdot \mathbf{n}_{\sigma, K}+\mu \sum_{K \in \mathcal{T}} \int_{K} \nabla \boldsymbol{u}^{n}: \nabla \boldsymbol{v} \mathrm{d} x \\
+\frac{\mu}{3} \sum_{K \in \mathcal{T}} \int_{K} \operatorname{div} \boldsymbol{u}^{n} \operatorname{div} \boldsymbol{v} \mathrm{d} x+T_{m}(\phi)=0, \text { for any } \boldsymbol{v} \in V_{h, 0}\left(\Omega ; R^{3}\right) \text { and } n=1, \ldots, N,
\end{gathered}
$$

where

$$
T_{c}(\phi)=h^{1-\varepsilon} \sum_{\sigma \in \mathcal{E}_{\text {int }}}|\sigma|\left[\varrho^{n}\right]_{\sigma, \boldsymbol{n}_{\sigma}}[\phi]_{\sigma \boldsymbol{n}_{\sigma}}, \quad T_{m}(\phi)=\sum_{\sigma \in \mathcal{E}_{\text {int }}}|\sigma|\left[\varrho^{n}\right]_{\sigma, \boldsymbol{n}_{\sigma}}\left\{\hat{\boldsymbol{u}}^{n}\right\}_{\sigma}[\hat{\phi}]_{\sigma, \boldsymbol{n}_{\sigma}}, \varepsilon \in[0,1),
$$


see Karlsen, Karper [19], Gallouet, Gastaldo, Herbin, Latché [15]. These terms are designed to provide the supplementary positive term

$$
h^{1-\varepsilon} \sum_{\sigma \in \mathcal{E}_{\text {int }}}|\sigma|\left[\varrho^{n}\right]_{\sigma, \boldsymbol{n}_{\sigma}}^{2},
$$

to the left hand side of the discrete energy identity (4.2). They contribute to the right hand side of the discrete relative energy (5.1) by supplementary terms whose absolute value is bounded from above by

$$
h^{(1-\varepsilon) / 2} c\left(M_{0}, E_{0}, \sup _{n=0, \ldots, N}\left\|r^{n}, \mathbf{U}^{n}, \nabla \mathbf{U}^{n}\right\|_{L^{\infty}\left(\Omega_{h} ; \mathbb{R}^{13}\right)}, \sup _{n=0, \ldots, N} \sup _{\sigma \in \mathcal{E} \operatorname{int}}\left[r^{n}\right]_{\sigma, \boldsymbol{n}_{\sigma}} / h\right) .
$$

Consequently, they give rise to the contributions at the right hand side of the approximate relative energy inequality (6.1) whose bound is

$$
h^{(1-\varepsilon) / 2} c\left(M_{0}, E_{0},\|r, \nabla r, \mathbf{U}, \nabla \mathbf{U}\|_{L^{\infty}\left(Q_{T} ; \mathbb{R}^{16}\right)}\right) .
$$

Similar estimates are true, if we replace in the numerical scheme everywhere classical upwind formula (3.4)

$$
\mathrm{Up}_{K}(q, \mathbf{u})=\sum_{\sigma \in \mathcal{E}(K)} q_{\sigma}^{\mathrm{up}} \boldsymbol{u}_{\sigma} \cdot \mathbf{n}_{\sigma, K}=\sum_{\substack{\sigma \in \mathcal{E}(K) \\ \sigma=K \mid L}}\left(q_{K}\left[\boldsymbol{u}_{\sigma} \cdot \mathbf{n}_{\sigma, K}\right]^{+}+q_{L}\left[\boldsymbol{u}_{\sigma} \cdot \mathbf{n}_{\sigma, K}\right]^{-}\right),
$$

by the modified upwind suggested in [11]:

$$
\mathrm{Up}_{K}(q, \mathbf{u})=\sum_{\substack{\sigma \in \mathcal{E}(K) \\ \sigma=K \mid L}} \frac{q_{K}}{2}\left(\left[\boldsymbol{u}_{\sigma} \cdot \boldsymbol{n}_{\sigma, K}+h^{1-\varepsilon}\right]^{+}+\left[\boldsymbol{u}_{\sigma} \cdot \boldsymbol{n}_{\sigma, K}-h^{1-\varepsilon}\right]^{+}\right)+\frac{q_{L}}{2}\left(\left[\boldsymbol{u}_{\sigma} \cdot \boldsymbol{n}_{\sigma, K}+h^{1-\varepsilon}\right]^{-}+\left[\boldsymbol{u}_{\sigma} \cdot \boldsymbol{n}_{\sigma, K}-h^{1-\varepsilon}\right]^{-}\right)
$$

where $\sigma=K \mid L \in \mathcal{E}_{\text {int }}$. We will finish by formulating the error estimate for the numerical problem (3.5), (10.1), (10.2) or for (3.5), (3.6), (3.7) with modified upwind (10.3).

Theorem 10.1. Let $\Omega, p,\left[r_{0}, \mathbf{V}^{0}\right],[r, V]$ satisfy assumptions of Theorem [3.1. Let $\left(\varrho^{n}, \mathbf{u}^{n}\right)_{n=0, \ldots, N}$ be a family of numerical solutions to the scheme (3.5), (10.1), (10.2) or to the scheme (3.5), (3.6), (3.7) with modified upwind (10.3), where $\varepsilon \in[0,1)$. Then error estimate (3.8) holds true with the exponent

$$
a=\min \left\{\frac{2 \gamma-3}{\gamma}, \frac{1-\varepsilon}{2}\right\} \text { if } \frac{3}{2} \leq \gamma<2, \quad a=\frac{1-\varepsilon}{2} \text { if } \gamma \geq 2 .
$$

\section{References}

[1] R.A. Adams. Sobolev spaces. Academic Press, New York, 1975.

[2] Y. Cho, H.J. Choe, and H. Kim. Unique solvability of the initial boundary value problems for compressible viscous fluids. J. Math. Pures. Appl., 83:243-275, 2004.

[3] R. Denk, M. Hieber, and J. Prüss. Optimal $L^{p}-L^{q}$-estimates for parabolic boundary value problems with inhomogenous data. Math. Z., 257:193-224, 2007.

[4] F. Brezzi and M. Fortin. Mixed and hybrid finite elements methods. Springer series in computational mathematics.

[5] C. Cancès, H. Mathis, and N. Seguin. Relative entropy for the finite volume approximation of strong solutions to systems of conservation laws. HAL : hal-00798287, 2013.

[6] M. Crouzeix and P. Raviart. Conforming and nonconforming finite element methods for solving the stationary Stokes equations. RAIRO Série Rouge, 7:33-75, 1973. 
[7] C. Dafermos. The second law of thermodynamics and stability. Arch. Rational Mech. Anal., 70:167$179,1979$.

[8] R. Danchin. On the solvability of the compressible Navier-Stokes system in bounded domains Nonlinearity 23: $383-407,2010$

[9] R. Eymard, T. Gallouët, M. Ghilani and R. Herbin. Error estimates for the approximate solutions of a non-linear hyperbolic equation given by a finite volume scheme, IMA J. Numer. Anal. 18(4): 563-594, 1998

[10] E. Feireisl, B. J. Jin, and A. Novotný. Relative entropies, suitable weak solutions, and weak-strong uniqueness for the compressible Navier-Stokes system. J. Math. Fluid Mech., 14(4):717-730, 2012.

[11] E. Feireisl, M. Michálek and T. K. Karper. Convergence of a numerical method for the compressible Navier-Stokes system on general domains. Preprint Inst. Math. Cz. Acad. Sci, 57, 2014.

[12] E. Feireisl and A. Novotný. Weak-strong uniqueness property for the full Navier-Stokes-Fourier system. Arch. Ration. Mech. Anal., 204(2):683-706, 2012.

[13] E. Feireisl, A. Novotný, and H. Petzeltová. On the existence of globally defined weak solutions to the Navier-Stokes equations. J. Math. Fluid Mech., 3(4):358-392, 2001.

[14] E. Feireisl, A. Novotný, and Y. Sun. Suitable weak solutions to the Navier-Stokes equations of compressible viscous fluids. Indiana Univ. Math. J., 60(2):611-631, 2011.

[15] T. Gallouët, L. Gastaldo, R. Herbin, and J.-C. Latché. An unconditionally stable pressure correction scheme for the compressible barotropic Navier-Stokes equations. M2AN Math. Model. Numer. Anal., 42(2):303-331, 2008.

[16] T. Gallouët, R. Herbin, and J.-C. Latché. A convergent finite element-finite volume scheme for the compressible Stokes problem. I. The isothermal case. Math. Comp., 78(267):1333-1352, 2009.

[17] T. Gallouët, R. Herbin, D. Maltese, and A. Novotný. Error estimate for a numerical approximation to the compressible barotropic navier-stokes equations. IMA Journal of Numerical Analysis, 2015 (published on-line).

[18] L. Gastaldo, R. Herbin, J.-C. Latché and N. Therme Consistency result of an explicit staggered scheme for the Euler equations Preprint, 2014.

[19] K. H. Karlsen and T. K. Karper. A convergent nonconforming finite element method for compressible Stokes flow. SIAM J. Numer. Anal., 48(5):1846-1876, 2010.

[20] T. K. Karper. A convergent FEM-DG method for the compressible Navier-Stokes equations. Numer. Math., 125(3):441-510, 2013

[21] N. V. Krylov. Parabolic equations with VMO coefficients in Sobolev spaces with mixed norms. $J$. Funct. Anal., 250(2):521-558, 2007.

[22] O. A. Ladyzhenskaya, V. A. Solonnikov, and N. N. Uralceva. Linear and qusilinear equations of parabolic type. AMS, Trans. Math. Monograph 23, Providence, 1968.

[23] P.-L. Lions. Mathematical topics in fluid mechanics. Vol. 2, volume 10 of Oxford Lecture Series in Mathematics and its Applications. The Clarendon Press, Oxford University Press, New York, 1998. Compressible models, Oxford Science Publications.

[24] B. Liu. The analysis of a finite element method with streamline diffusion for the compressible Navier-Stokes equations. SIAM J. Numer. Anal., 38(1):1-16 (electronic), 2000. 
[25] B. Liu. On a finite element method for three-dimensional unsteady compressible viscous flows. Numer. Methods Partial Differential Equations, 20(3):432-449, 2004.

[26] Y. Sun, C. Wang, Z. Zhang A Beale-Kato-Majda blow-up criterion for the 3-D compressible Navier-Stokes equations J. Math. Pures Appl., 95 (1): 36-47, 2011

[27] R. Temam. Navier-Stokes equations, volume 2 of Studies in Mathematics and its Applications. North-Holland Publishing Co., Amsterdam, third edition, 1984. Theory and numerical analysis, With an appendix by F. Thomasset.

[28] A. Valli and M. Zajaczkowski. Navier-Stokes equations for compressible fluids: Global existence and qualitative properties of the solutions in the general case. Commun. Math. Phys., 103:259-296, 1986.

[29] C. Rhode and V. Yovanovic Finite volume schemes for Friedrichs systems in multiple space dimensions: a priori and a posteriori estimates. Numer. Meth. P.D.E., 21(1):104-131, 2005.

[30] J.P. Villa and P. Villedieu Convergence of an explicite finite volume scheme for first order symmetric systems. Numer. Math., 94(3):573-602, 2003.

[31] V. Yovanovic. An error estimate for a numerical scheme for the compressible Navier-Stokes system. Kragujevac J. Math., 30(1):263-275, 2007. 